\title{
Monolithic Integration of GaN HEMT with Silicon MOS Technology
}

by

Pietro Natale Alessandro Chyurlia

\author{
A Dissertation submitted to \\ the Faculty of Graduate and Postdoctoral Affairs \\ in partial fulfilment of \\ the requirements for the degree of \\ Doctor of Philosophy
}

Ottawa-Carleton Institute for

Electrical and Computer Engineering

Department of Electronics

Carleton University

Ottawa, Ontario, Canada

March 152011

Copyright (c)

2011 - Pietro Natale Alessandro Chyurlia 
Library and Archives

Canada

Published Heritage

Branch

395 Wellington Street

Ottawa ON K1A ON4

Canada
Bibliothèque et

Archives Canada

Direction du

Patrimoine de l'édition

395 , rue Wellington

Ottawa ON K1A ON4

Canada
Your file Votre référence

ISBN: 978-0-494-81540-3

Our file Notre référence

ISBN: $978-0-494-81540-3$
NOTICE:

The author has granted a nonexclusive license allowing Library and Archives Canada to reproduce, publish, archive, preserve, conserve, communicate to the public by telecommunication or on the Internet, loan, distribute and sell theses worldwide, for commercial or noncommercial purposes, in microform, paper, electronic and/or any other formats.

The author retains copyright ownership and moral rights in this thesis. Neither the thesis nor substantial extracts from it may be printed or otherwise reproduced without the author's permission.
AVIS:

L'auteur a accordé une licence non exclusive permettant à la Bibliothèque et Archives Canada de reproduire, publier, archiver, sauvegarder, conserver, transmettre au public par télécommunication ou par l'Internet, prêter, distribuer et vendre des thèses partout dans le monde, à des fins commerciales ou autres, sur support microforme, papier, électronique et/ou autres formats.

L'auteur conserve la propriété du droit d'auteur et des droits moraux qui protège cette thèse. $\mathrm{Ni}$ la thèse ni des extraits substantiels de celle-ci ne doivent être imprimés ou autrement reproduits sans son autorisation.
In compliance with the Canadian Privacy Act some supporting forms may have been removed from this thesis.

While these forms may be included in the document page count, their removal does not represent any loss of content from the thesis.
Conformément à la loi canadienne sur la protection de la vie privée, quelques formulaires secondaires ont été enlevés de cette thèse.

Bien que ces formulaires aient inclus dans la pagination, il n'y aura aucun contenu manquant.

\section{Canadä}


The undersigned recommend to the Faculty of Graduate and Postdoctoral Affairs acceptance of the thesis

\title{
Monolithic Integration of GaN HEMT with Silicon MOS Technology
}

\author{
Submitted by \\ Pietro Chyurlia, B.Eng., M.A.Sc. \\ In partial fulfillment of the requirements for the degree of \\ Doctor of Philosophy \\ in Electrical Engineering
}

Professor Garry Tarr, Co-Supervisor

$\overline{\text { Adjunct Professor Jennifer Bardwell, Co-Supervisor }}$

Dr. Malcolm Stubbs, External Examiner

Professor Qi-Jun

Chair, Department of Electronics

Ottawa-Carleton Institute for Electrical and Computer Engineering

Carleton University

Department of Electronics

2011 


\section{Abstract}

This work reports on the first monolithic integration of silicon-based n-channel metaloxide semiconductor (NMOS) transistors with GaN high electron mobility transistors (HEMT) on silicon $<111>$ and $<110>$ oriented silicon substrates. A windowed growth technique based on differential epitaxy was developed, which allows for GaN HEMT layers to be grown on silicon substrates while preserving regions of atomically smooth silicon for MOS fabrication. A low thermal budget fabrication process was designed that includes $2.5 \mu \mathrm{m}$ long isolated NMOS devices alongside $0.8 \mu \mathrm{m}$ long dual gate GaN HEMTs. Isolated NMOS devices with a gate oxide breakdown of 12.6 $\mathrm{MV} / \mathrm{cm}$ and no gate leakage were successfully fabricated and tested. The performance of the GaN HEMT layers was comparable to that achieved for GaN layers grown on sapphire substrates. The saturation drain current exceeded $0.8 \mathrm{~mA} / \mathrm{mm}$ with minimal current collapse, a drain breakdown voltage of $200 \mathrm{~V}$ and a transconductance of $191 \mathrm{mS} / \mathrm{mm}$. 
To my father 


\section{Acknowledgments}

I would like to thank my thesis supervisor and mentor, Dr. Garry Tarr, for all the support and assistance he has given me not only for the duration of my thesis, but for the past 10 years at Carleton University. How many thesis supervisors let you have crazy parties in their house? Not many...thanks Garry. Next I need to thank Dr. Jennifer Bardwell who has been incredibly supportive during the past seven years, both with respect to research as well as just being there to talk to about life and all its issues.

This research would have been impossible to complete without the dedicated work of my two wafer growers, Dr. Fabrice Semond and Dr. Haipeng Tang. Their hard work in the field and co-operation in developing our windowed growth technique was invaluable. They also worked many extra hours to give me enough wafers to run all of my experiments.....in addition to many that either exploded on me or turned into potato chips during fabrication. Dr. Tim Lester, whose office I would find myself in after anything started going wrong with my process, has been a wonderful encyclopedia of knowledge with respect to device processing. Without his training in the lab and expertise in photolithography, this project could not have been successfully completed.

Fabrication support was given by the following people: Carol Adams, Rob VanDusen, Angela Burns, Rick Adams, Nagui Mikhail, Stephen Rolfe, Dan Roth, Hue Tran, Jeff Fraser, Phil Waldron, Rongzhu Wang, Margaret Buchanan, Souffien Haffouz, Jeff 
Fraser, and Simona Moisa. All of them were a huge help over past few years and this work could not have succeeded without them. I would like to personally recognize Carol for being my second mom away from home in the lab. Carol took me under her wing ten years ago showing me around the lab. Since then we have shared many stories about her life, and she has given me sound advice about mine.

Dr. Steve McGarry and Rony Amaya were also instrumental in making this work a success. Rony, thank you for all those circuit design meetings and ideas we had in your office; your expertise in circuit design and layout was especially useful to me. Steve, you were always there when I had a hiccup with my research and were always up for a chat whether it was in your office, the coffee shop, or your favourite spot: the bar. I would also like to thank Dr. Alan Harrison and Dr. Robert Surridge from Gain Microwave; they were instrumental in helping me set up the Carleton load-pull system and IC-CAP for device modelling and simulation.

My dear friend Suzette Lee is thanked for being my primary proofreader for this work. Thanks to her, I am too familiar with the concept of the Oxford comma. I would like to thank my awesome family, especially my mom and sister for keeping me sane, and my father for being a constant source of motivation throughout my studies. Finally, thanks to my friends who have been by my side throughout life's ups and downs. 


\section{Table of Contents}

Abstract $\quad$ iv

Acknowledgments $\quad$ vi

Table of Contents viii

List of Tables $\quad$ xii

List of Figures $\quad$ xiii

Nomenclature $\quad$ xvi

1 Introduction $\quad 1$

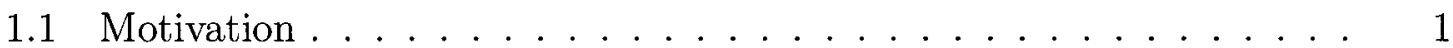

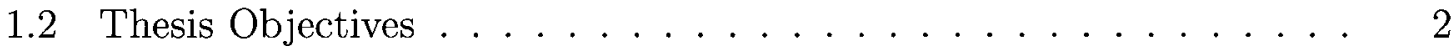

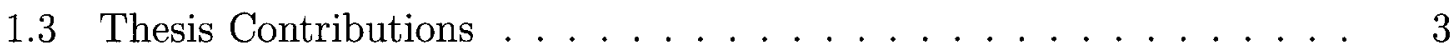

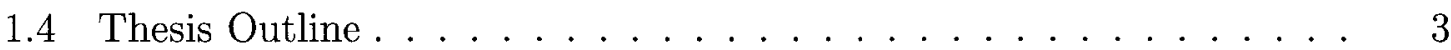

2 Background $\quad 5$

2.1 Silicon Substrates . . . . . . . . . . . . . . . 6

2.1.1 Growth on $<110>$ Silicon .............. 9

2.2 GaN HEMT Technology Overview . . . . . . . . . . . . . . 10

2.2.1 Growth and Process Description . . . . . . . . . . . 10 
2.2 .2 HEMT Operation . . . . . . . . . . . . . . . . 12

2.3 GaN Innovations . . . . . . . . . . . . . . . . . . . . . . . . 13

2.4 Low Temperature MOS processing . . . . . . . . . . . . . . 14

2.4.1 Low Temperature Oxidation of Silicon . . . . . . . . . 15

2.5 Towards an Integrated Technology . . . . . . . . . . . . . 16

3 Integration Strategy and Considerations 18

3.1 Growth Considerations . . . . . . . . . . . . . . . . . 18

3.2 Processing Background . . . . . . . . . . . . . . . . . . 19

3.3 Thermal Budget Considerations . . . . . . . . . . . . . . . . 21

3.4 Thermal Performance Considerations . . . . . . . . . . . . . . 23

3.5 Final Approach . . . . . . . . . . . . . . . . . . . 24

4 Windowed Epitaxial GaN Growth on Silicon 26

4.1 Primary Challenges . . . . . . . . . . . . . . . . . 26

4.2 Windowed Growth $\ldots \ldots \ldots \ldots \ldots \ldots$

4.2 .1 Detailed Growth Process . . . . . . . . . . . . . . . 29

4.2.2 Windowed Growth Wafer Properties . . . . . . . . . 33

4.3 Silicon Surface Properties $\ldots \ldots \ldots$. . . . . . . . . 37

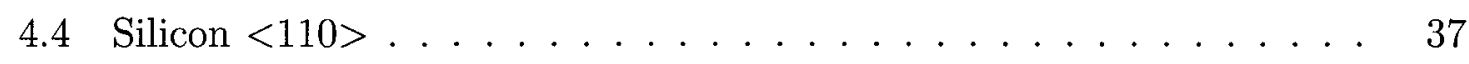

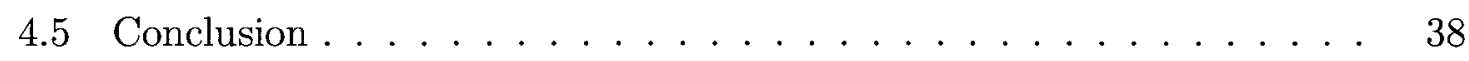

5 Process Design $\quad 40$

$5.1 \quad$ NMOS Design . . . . . . . . . . . . . . . . . . . . . . . . 41

$5.1 .1 \quad$ Enclosed MOS Devices . . . . . . . . . . . . . . . . . 44

5.1 .2 Electrical Characteristics . . . . . . . . . . . . . 46

5.2 GaN Devices $\ldots \ldots \ldots \ldots \ldots \ldots \ldots$

5.3 Metallization and Passives . . . . . . . . . . . . . . 48 
5.3.1 Metallization .................. 48

5.3.2 Passive Components .................. 48

5.4 Development of a Gold-Free Ohmic Contact . . . . . . . . . . . . . 49

5.4.1 Fabrication and Characterization of Ohmic Contacts . . . . 50

5.4 .2 Stress in Ohmic Contacts .............. . 51

5.5 Mask Layout . . . . . . . . . . . . . . . . . . 52

5.6 Second Iteration of MOS Design . . . . . . . . . . . . . 55

5.7 Summary ............................. 57

6 MOS Device Performance $\quad 65$

6.1 First Run of Enclosed MOS Devices . . . . . . . . . . . 65

6.2 Second Process Iteration; Higher Temperature Oxidations, Isolated De-

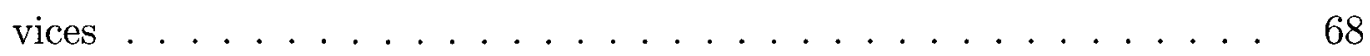

6.2.1 Device Isolation in Batch 488L . . . . . . . . . . . 71

6.3 CV Analysis of MOS Gate Dielectics . . . . . . . . . . . . . 71

6.4 Oxide Breakdown Measurements . . . . . . . . . . . . 73

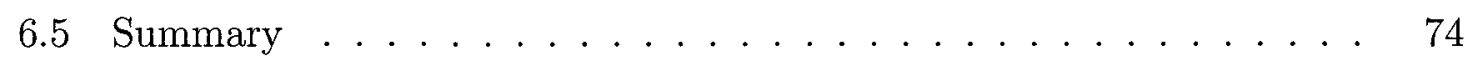

7 GaN Device Performance $\quad 76$

7.1 Measurement Summary .................. 76

7.2 Gate Leakage . . . . . . . . . . . . . . . 78

7.3 Current Collapse .................... 80

7.4 Breakdown Characteristics . . . . . . . . . . . . 81

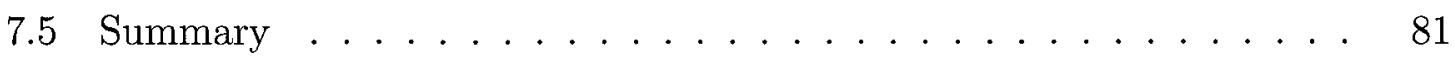

8 Conclusions and Future Work $\quad 83$

8.1 Future Directions ...................... 83

8.2 Summary of Contributions . . . . . . . . . . . . . 84 
Appendix A Tsuprem Input File for Original nMOS Design

Appendix B Medici Input file used to calculate MOS device properties 101

Appendix C Process Sheet for Fabrication Process

114

C.1 Enclosed Process Flow . . . . . . . . . . . . . . . . 114

Appendix D CV calculations 


\section{List of Tables}

2.1 Comparison of semiconductor materials' power properties . . . . . . . 6

2.2 Comparison of substrates available for GaN epitaxy . . . . . . . . . . 7

2.3 Current power handling records for $\mathrm{GaN}$ on $\mathrm{Si}<111>$ substrates . . . 8

2.4 Comparison of properties for state-of-the-art GaN HEMTs on $\mathrm{SiC}$ vs Si. Values quoted at frequency of $4 \mathrm{GHz}[7][21] \ldots . . \ldots 9$

4.1 Comparison of the properties of the best C-doped and non C-doped

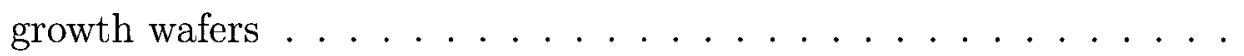

4.2 Comparison of the properties of 110 windowed growths. 479L batch grown at $\mathrm{NRC}, 455 \mathrm{~L}$ batch grown at CRHEA. Note that due to the windowed growth geometry, numbers are not exact, but can be used to compare between substrates . . . . . . . . . . . 38

5.1 Ohmic contact results as measured by TLM patterns following fabrication 51

5.2 Summary of P-well retrograde well implant parameters . . . . . . . . 56

6.1 Threshold voltage comparison for batch 464L . . . . . . . . . 67

6.2 CMOS device summary. $12.5 \mu \mathrm{m}$ and $2.5 \mu \mathrm{m}$ gate length data given 69

6.3 Oxidation thickness summary using test wafers for each batch. . . . 73

7.1 Summary of GaN device performance for successful runs. X denotes a value that could not be measured. . . . . . . . . . . . . 77

7.2 Breakdown Voltage vs Gate-Drain Spacing . . . . . . . . . 81 


\section{List of Figures}

2.1 Typical GaN HEMT layer structure for growth on silicon $<111>$ sub-

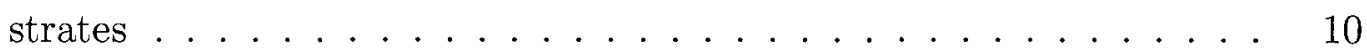

2.2 Conduction band diagram of GaN HEMT [25] . . . . . . . . . 11

2.3 Non-passivated (left) and passivated (right) device IV curves showing mitigation of current collapse using pulsed measurements on sapphire substrates (pulsed measurement represented by squares, continuous sweep represented by circles). . . . . . . . . . . . . . . 13

3.1 Primary steps for both CMOS and GaN processes . . . . . . . . . 20

3.2 Optical and SEM pictures of sample annealed at $1000^{\circ} \mathrm{C} \ldots 22$

3.3 Cross section of envisioned co-integration process . . . . . . . . . . 24

4.1 a. SEM image of AlGaN/GaN heterostructure ICP etched down to the silicon surface. Note the poor quality of the silicon surface which includes both cracks and surface pits. b. FIB Section of wafer showing cracks originating between the stress relief layers. c. Optical microscope image of an attempted smoothing of microcracks using TMAH.

4.2 Comparison of growth techniques $\ldots \ldots \ldots \ldots \ldots$

4.3 Wafer before MBE growth step. Note smooth silicon surface available for epitaxy. . . . . . . . . . . . . . . . . . . . 30

4.4 Windowed growth images $\ldots \ldots \ldots \ldots \ldots \ldots \ldots$

4.5 GaN layer structure for growth on silicon $<111>$ substrates $\ldots \ldots 32$ 
4.6 CV carrier concentration profile in the 2DEG and GaN buffer . . . . 34

4.7 SIMS profile comparing oxygen and carbon impurity concentrations for standard and windowed growths . . . . . . . . . . . . 36

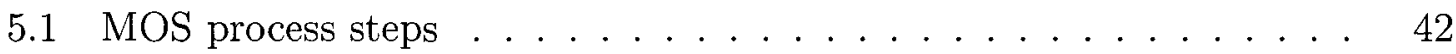

5.2 Doping profiles from Tsuprem4 simulation . . . . . . . . . . 43

5.3 Cross-Section of enclosed nMOS device . . . . . . . . . . . 45

5.4 Layout of an enclosed NMOS device . . . . . . . . . . . 45

5.5 Medici simulations of MOS electrical characteristics for a $2.5 \mu \mathrm{m}$ long

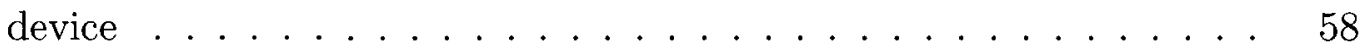

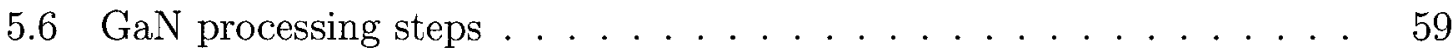

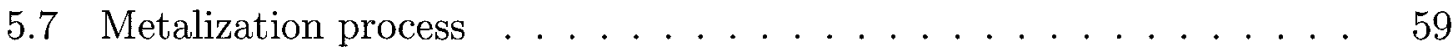

5.8 SEM micrograph of crack formation in the gate periphery of a device due to ohmic contact anneal (sample 456LB) . . . . . . . . . 60

5.9 First mask layout showing MOS region (bottom left) as well as other

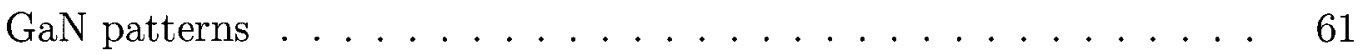

5.10 Enclosed mask layout showing MOS region (bottom left) as well as

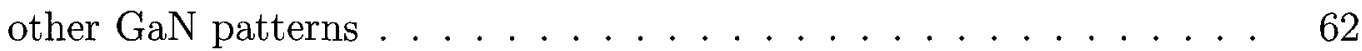

5.11 Revised doping profiles from Tsuprem4 simulation . . . . . . . . . 63

5.12 MOS cross Sections showing both isolation techniques . . . . . . . . 63

5.13 SEM images of integrated GaN HFET and MOS devices . . . . . . . 64

6.3 TSuprem4 simulation of P-Well implantation with $200 \mathrm{~nm}$ thick screen oxide used rather than $20 \mathrm{~nm} \ldots \ldots$. . . . . . . . . . 70

6.4 CV curves for gate oxides on test wafers using CV dots . . . . . . . 72

6.5 Oxide breakdown curves for test oxides for batch $485 \mathrm{~L}$ and $488 \mathrm{~L}$ $(<111>$ oriented $) \ldots \ldots . \ldots . \ldots . \ldots 74$

7.1 DC measurements for dual-gate AlGaN/GaN HEMT on sample 464LA 78

7.2 Maximum forward current vs. device width for sample 488LA . . . . 79 
7.3 Gate leakage comparison for C-doped 464LC sample (dashed) vs non C-doped 464LA sample . . . . . . . . . . . . . . . . . . 79

7.4 Pulsed (dashed) and non-pulsed (solid) $\mathrm{J}_{D S}$ vs $\mathrm{V}_{D S}$ curves for wafer $464 \mathrm{LA} \ldots \ldots \ldots \ldots$ 


\section{Nomenclature}

2-DEG : 2-Dimensional Electron Gas

a-Si : Amorphous Silicon

ADC : Analog-to-Digital Converter

ADS : Advanced Design System

AES : Auger Electron Spectroscopy

AFM : Atomic Force Microscopy

AlN : Aluminum Nitride

BOE : Buffered Oxide Etch

BPSG : Borophosphosilicate Glass

CAIBE : Chemically-Assisted Ion Beam Etch

CMOS: Complementary Metal-Oxide Semiconductor

CV : Capacitance-Voltage

FIB : Focussed Ion Beam

GaAs : Gallium Arsenide

GaN : Gallium Nitride

HEMT : High Electron Mobility Transistor

HFET : Heterostructure Field Effect Transistor

ICP : Inductively Coupled Plasma

LDMOS : Lateral Double-diffused MOSFET 
LOCOS : Local Oxidation of Silicon

LPCVD : Low Pressure Chemical Vapour Deposition

MBE : Molecular Beam Epitaxy

MESFET: Metal Epitaxial Semiconductor Field Effect Transistor

MiM : Metal-Insulator-Metal

MOCVD : Metal-Organic Chemical Vapour Deposition

MOS : Metal Oxide Semiconductor

MOScap: MOS Capacitor

NMOS : N-type Metal Oxide Semiconductor

NRC : National Research Council

PAE : Power Added Efficiency

PECVD : Plasma-Enhanced Chemical Vapour Deposition

PMOS : P-type Metal Oxide Semiconductor

RIE : Reactive Ion Etch

RMS : Root Mean Square

RTA : Rapid Thermal Anneal

SEM : Scanning Electron Microscope

$\mathrm{SiC}$ : Silicon Carbide

SiN : Silicon Nitride

SIMS : Secondary Ion Mass Spectroscopy

SOI : Silicon-on-Insulator

TLM : Transmission Line Model

TMAH : Tetra-Methyl-Ammonium-Hydroxide

XPS : X-ray Photoluminescence Spectroscopy 


\section{Chapter 1}

\section{Introduction}

\subsection{Motivation}

Gallium Nitride $(\mathrm{GaN})$ transistors are an emerging technology that may soon replace GaAs devices for high-power, high-frequency applications [1]. This is due to the ability of GaN to withstand high voltage and temperatures, while maintaining high electron mobility. In order to grow heteroepitaxial GaN high electron mobility transistor (HEMT) layers, the lattice constants of the substrate and epitaxial layers must be matched in order to reduce stresses on the layers, and lower the defect density. The two primary substrates used for GaN in its development were sapphire and silicon carbide ( $\mathrm{SiC})$. The advantage of sapphire is that it is reasonably inexpensive compared to $\mathrm{SiC}$ wafers. However, because of the low thermal conductivity of sapphire, devices grown on $\mathrm{SiC}$ experience far superior performance due to the reduction of self-heating effects. While silicon carbide has a much higher thermal conductivity, allowing for high-performance devices, the high cost of the material makes it economically challenging to use.

Advances in epitaxy have allowed for the GaN layers to be grown on silicon $<111>[2]$, off-cut $<100>[3,4]$ and $<110>$ [5] surfaces. The low cost of silicon compared to that of $\mathrm{SiC}$ makes it the most advantageous substrate to use, even though it 
has a worse lattice mismatch to GaN and lower thermal conductivity than competing $\mathrm{SiC}$ substrates. Additionally, the thermal conductivity of silicon is far superior to that of sapphire substrates. Advances in epitaxy have allowed for almost stress-free and defect-free commercial GaN layers to be grown on silicon, and devices that have exceeded the $7 \mathrm{~W} / \mathrm{mm}$ power level at $10 \mathrm{GHz}$ and $12 \mathrm{~W} / \mathrm{mm}$ at $2 \mathrm{GHz}$ have been reported $[6,7]$.

The growth of GaN on silicon substrates presents an opportunity for the monolithic integration of silicon microelectronics with GaN HEMT structures. This integration will allow for smaller, faster, and less expensive chips, which can combine power transistors with other logic typically needed for high frequency transmit modules. In addition, the use of GaN power devices with silicon microelectronics lends itself to sensing or routing applications with built-in logic or read-out circuitry.

\subsection{Thesis Objectives}

The primary objective of this work is to fabricate a demonstration monolithic chip consisting of both silicon metal-oxide-semiconductor (MOS) devices and GaN power devices on a silicon $\langle 111\rangle$ substrate. Overcoming the fabrication challenge of developing a joint process which yields both high-performance GaN devices, as well as MOS circuitry, is the primary goal of this work.

The National Research Council of Canada (NRC) has a well-established GaN research group, which focuses on the growth and fabrication of AlGaN/GaN HEMT devices. A mature GaN HEMT process that has already been developed at NRC has been used in combination with a custom MOS process to accomplish the integration. The MOS fabrication took place at Carleton University's Microfabrication lab. This facility, which specializes in silicon devices, is capable of making devices with a minimum feature size of $2.5 \mu \mathrm{m}$ with in-house photomasks, and $0.8 \mu \mathrm{m}$ with industrial 
quality chrome photomasks. Additionally, an established nMOS process is run in this lab that has been adapted for use in this work. Combining the resources of both these facilities provided a unique advantage in pursuing this innovative project.

\subsection{Thesis Contributions}

The following is a list of the primary contributions in this work.

- Development of a windowed growth technique based on differential epitaxy which preserves an atomically smooth silicon surface for both $<111\rangle$ and $<110>$ oriented surfaces following GaN growth.

- First demonstration of monolithic integration of MOS and AlGaN/GaN HEMT devices on silicon $<111>$ and $<110>$ substrates.

- Development of a low-temperature, gold-free joint MOS/GaN process resulting in devices of both types demonstrating good performance.

\subsection{Thesis Outline}

This thesis begins with a review chapter giving a summary the current state of gallium nitride technology, as well as some important developments with state-of-the-art MOS that are relevant to this work. Chapter 3 summarizes the challenges that need to be overcome in order to successfully integrate both types of devices on one chip. The methodology used in this work is based on this discussion and is presented at the end of this chapter.

Chapter 4 presents a detailed description of the windowed growth procedure, which is the critical technique developed to allow for this work to be successful. The following chapter presents the fully-detailed fabrication process for integrating the 
MOS and GaN devices. TSuprem4 and Medici simulations are used to design the MOS flow [8]. The GaN process is adapted from the existing NRC process to be compatible with the MOS processing.

The results and device performance for both MOS and GaN devices are given in Chapters 6 and 7. Recommendations for improvement of the process are given as a discussion, which is followed by a conclusion that summarizes this work. 


\section{Chapter 2}

\section{Background}

Gallium Nitride HEMT technology is becoming the front-runner for future highpower, high-voltage, and medium frequency applications such as cell phone infrastructure (base stations), military communications, as well as automotive and other general power conversion devices [9]. The combination of a high breakdown field, large bandgap, high mobility, and the ability to operate at elevated temperatures make GaN an ideal material for high-voltage and high-power applications. Table 2.1 gives a summary of the properties of common semiconductor materials relevant for power device applications. Diamond, a material that has a higher Johnson Figure of Merit, is plagued by poor doping control and difficulty in forming ohmic contacts [10].

The ability to form a HEMT using a GaN/AlGaN heterostructure is advantageous for several reasons; HEMT devices typically have better noise performance than MESFETs (MEtal Semiconductor Field-Effect Transistor) [9], and the mobility is high due to the formation of a 2-dimensional electron gas (2DEG), which reduces scattering effects by confining electrons away from scattering centres [9]. This makes GaN a superior choice over $\mathrm{SiC}$, which to date has not demonstrated HEMTs.

The primary competitor to GaN for high-power RF applications is silicon LDMOS (Lateral Double-diffused MOSFET) technology due to its low cost. However, silicon LDMOS devices are being pushed to ultimate physical limits by present power 


\begin{tabular}{|l||c|c|c|c|c|}
\hline Material & $\mathrm{Si}$ & $\mathrm{GaAs}$ & $\mathrm{SiC}$ & $\mathrm{GaN}$ & Diamond \\
\hline Bandgap $(\mathrm{eV})$ & 1.1 & 1.42 & 3.26 & 3.4 & 5.45 \\
Electron Mobility $\left(\mathrm{cm}^{2} / \mathrm{Vs}\right)$ & 1350 & 8500 & 700 & $1000-2000$ & 1900 \\
Saturation Velocity $\left(10^{7} \mathrm{~cm} / \mathrm{s}\right)$ & 1.0 & 1.0 & 2.0 & 2.5 & 2.7 \\
Critical Breakdown Field $(\mathrm{MV} / \mathrm{cm})$ & 0.3 & 0.4 & 3.0 & 3.3 & 5.6 \\
Thermal Conductivity $(\mathrm{W} / \mathrm{cm} \cdot \mathrm{K})$ & 1.5 & 0.43 & $3.3-4.5$ & 1.3 & 20 \\
Johnson Figure of Merit $\left(E_{b r} V_{\text {sat }} / 2 \pi\right)$ & 1 & 2.7 & 20 & 27.5 & 50 \\
Dielectric Constant $\left(\varepsilon_{r}\right)$ & 11.9 & 13.1 & 10 & 9.0 & 5.5 \\
HEMT structures & no & yes & no & yes & no \\
\hline
\end{tabular}

Table 2.1: Comparison of semiconductor materials' power properties $[9][10]$

and frequency demands, and hence require high-complexity modules which include linearization circuitry. GaN devices offer inherently superior linearity compared to LDMOS, reducing the need for added linearization techniques. This allows for reduced complexity, and therefore, lower cost modules [10].

\subsection{Silicon Substrates}

Initial demonstrations of GaN devices were grown on sapphire due to its low cost and ready availability of 2 and 4 inch substrates (Table 2.2). The primary drawback of sapphire is the poor thermal conductivity of the material, resulting in excessive heating of HEMT devices, which in turn hampers performance.

Silicon-carbide ( $\mathrm{SiC}$ ) was the next substrate to be used, and is still the best choice for high performance due to its extremely high thermal conductivity. Drawbacks of $\mathrm{SiC}$ are the high cost and limited diameter of the substrates.

The growth of GaN HEMT structures on high-resistivity silicon <111>-oriented surfaces was developed in 1999 by Chumbes et al. at Cornell [13]. Although silicon has 


\begin{tabular}{|l||c|c|c|c|c|}
\hline Substrate & GaN & AlN & Si & SiC & Sapphire \\
\hline Thermal Conductivity $(W / \mathrm{cm} \cdot K)$ & 1.4 & 3 & 1.5 & 3 & 0.5 \\
Resistivity $(\Omega \cdot \mathrm{cm})$ & $>10^{4}$ & $>10^{13}$ & $>10^{4}$ & $>10^{4}$ & $>10^{8}$ \\
Diameter used for Epitaxy $(\mathrm{in})$ & 2 & 2 & $2-8$ & $2-3$ & $2-4$ \\
Lattice Mismatch to GaN (\%) & 0 & 2.5 & 16.9 & 3.5 & 16 \\
Thermal Expansion Mismatch to GaN & 0 & 33 & 116 & 18.9 & -25.4 \\
$\left(\left(\alpha_{L(G a N)}-\alpha_{L(x)}\right) / \alpha_{L(x)}\right)$ & & & & & \\
Price & high & high & low & high & low \\
\hline
\end{tabular}

Table 2.2: Comparison of substrates available for GaN epitaxy [11] [12]

a large lattice mismatch with $\mathrm{GaN}$, as well as a large thermal expansion mismatch, it provides several advantages over sapphire and $\mathrm{SiC}$ substrates. Table 2.2 summarizes the properties of various substrates for GaN epitaxy.

The primary advantage of using silicon as a substrate is the low cost, resulting from the years of microelectronics development using this material. Recent developments in epitaxy have also resulted in growth on 8-inch silicon substrates, compared to the maximum of 3 inches for $\mathrm{SiC}$ and 4 inches for sapphire [14]. This represents a step towards lower-cost GaN-on-silicon power devices.

Epitaxial growth of $\mathrm{GaN}$ on $\mathrm{Si}$ is challenging due to the large thermal expansion and lattice mismatches, but techniques to overcome this have been developed over the past decade, resulting in increased performance and reliability. Table 2.3 summarizes how the power handling capabilities of GaN HEMTs on silicon have improved over time.

The performance of GaN devices grown on $\mathrm{SiC}$ will always exceed that of $\mathrm{GaN}$ devices grown on silicon, but at a much higher cost. This is primarily due to the superior heat dissipation abilities of the substrate. The financial advantage of using silicon will certainly push SiC-based devices to the regime in which performance must 


\begin{tabular}{|c|c|c|c|}
\hline Year & Frequency & Power Density & Group \\
\hline 1999 & $4 \mathrm{GHz}$ & $0.5 \mathrm{~W} / \mathrm{mm}$ & Cornell \\
2002 & $4 \mathrm{GHz}$ & $1 \mathrm{~W} / \mathrm{mm}$ & CRHEA-CNRS/IEMN \\
2003 & $2 \mathrm{GHz}$ & $6.6 \mathrm{~W} / \mathrm{mm}$ & CRHEA-CNRS/Daimler Chrysler \\
2004 & $10 \mathrm{GHz}$ & $7 \mathrm{~W} / \mathrm{mm}$ & Triquint/Picogiga (CRHEA patent) \\
2004 & $4 \mathrm{GHz}$ & $12 \mathrm{~W} / \mathrm{mm}$ & Nitronex \\
2005 & $2 \mathrm{GHz}$ & $10.2 \mathrm{~W} / \mathrm{mm}$ & Nitronex \\
2006 & $18 \mathrm{GHz}$ & $5.1 \mathrm{~W} / \mathrm{mm}$ & IEMN/Picogiga \\
2010 & $10 \mathrm{GHz}$ & $6.1 \mathrm{~W} / \mathrm{mm}$ & Triquint \\
\hline
\end{tabular}

Table 2.3: Current power handling records for GaN on $\mathrm{Si}<111>$ substrates [13] [15] [16] [6] [7] [17] [18] [19]

be maximized. Power amplifiers delivering up to $368 \mathrm{~W}$ from a $60 \mathrm{~V}$ supply at $2 \mathrm{GHz}$ have been recently reported using $\mathrm{GaN}$ on silicon $<111>$ substrates [17]. However, much higher power densities have been realized with $\mathrm{SiC}$ substrates. Table 2.4 compares the power properties between two state-of-the-art GaN HEMTs, one grown on $\mathrm{SiC}$ and the other on Si. Additionally, SiC substrates may offer better stability as the lattice mismatch to the substrate is lower. The breakdown voltage may also be higher compared to silicon as there is no available leakage path through the substrate. However, breakdown voltages of greater than $1000 \mathrm{~V}$ have also been reported on silicon substrates [20].

Growth of GaN HEMT structures has recently been developed on off-cut <100> as well as $<110>$ oriented silicon substrates [3] [4] [5]. This breakthrough is extremely relevant to this work as $<100>$ and $<110>$ are the preferred orientations for silicon microelectronics due to the lower number of interface states compared to $<111>$. Additionally, $<110>$ oriented substrates are of increasing interest for state-of-theart CMOS processes due to the large increase in hole mobility in strained devices compared to $<100>$ oriented substrates, which have been the conventional choice in 


\begin{tabular}{|c|c|c|}
\hline Property & $\mathrm{SiC}(\mathrm{Cree})$ & $\mathrm{Si}$ (Nitronex) \\
\hline Power Density at $4 \mathrm{GHz}$ & $32 \mathrm{~W} / \mathrm{mm}$ & $12 \mathrm{~W} / \mathrm{mm}$ \\
Sheet Resistance & $265 \mathrm{Ohms} / \mathrm{sq}$ & $300 \mathrm{Ohms} / \mathrm{sq}$ \\
Sheet Charge x Mobility Product & $2.35 \times 10^{16}(\mathrm{Vs})^{-1}$ & $2.1 \times 10^{16}(\mathrm{Vs})^{-1}$ \\
Gain & $16.8 \mathrm{~dB}$ & $15.3 \mathrm{~dB}$ \\
Breakdown Voltage & Not Provided & $>200 \mathrm{~V}$ \\
\hline
\end{tabular}

Table 2.4: Comparison of properties for state-of-the-art GaN HEMTs on SiC vs Si. Values quoted at frequency of $4 \mathrm{GHz}$ [7] [21]

the past [22].

\subsubsection{Growth on $<110>$ Silicon}

$<110>$-oriented silicon substrates are of increasing interest for this work as GaN epilayers can be grown on them without having to use off-cut, specially made substrates, unlike the $<100>$ orientation. Additionally, $<110>$-oriented Si substrates are currently being explored for state-of-the-art CMOS fabrication as the hole mobility has been shown to increase by $160 \%$ for pMOS devices compared to conventional $<100>-$ oriented substrates [22]. Meanwhile, the nMOS mobility is only reduced by between $49 \%$ and $34 \%$ depending on the direction of current flow [22]. Although the nMOS mobility, and hence current drive, is reduced, the large increase in pMOS performance reduces the disparity in speed between nMOS and pMOS devices, which may allow for an overall speed increase for logic applications. The primary concern for $<110>$ oriented substrates, especially for RF and microwave applications, is the higher noise and gate leakage compared to $\langle 100\rangle$ silicon due to more electron-trapping states being present at the $\mathrm{Si} / \mathrm{SiO}_{2}$ interface. However, with the movement towards high$\mathrm{k}$ dielectrics, which are often deposited rather than grown, the problems caused by the higher density of states can be mitigated, eliminating a major obstacle for this 
technology [23].

\subsection{GaN HEMT Technology Overview}

GaN High Electron Mobility Transistors make use of a Schottky gate to control a device channel, formed by a vertical heterostructure of $\mathrm{Al}_{x} \mathrm{Ga}_{1-x} \mathrm{~N}$ and $\mathrm{GaN}$ layers. The device channel in this case is a 2-dimensional electron gas (2-DEG), which is formed at the interface of the $\mathrm{GaN}$ and AlGaN layers. A combination of the spontaneous polarization and piezoelectric polarization of AlGaN induces the 2DEG, which is trapped by band offset [24].

\begin{tabular}{|c|c|}
\hline Source & \multicolumn{1}{|c|}{ Gate } \\
\hline BlGaN Barrieg $-25 \% \mathrm{Al}$ \\
\hline GaN Buffer (C-doped) $\sim 1-3 \mathrm{um}$ \\
\hline AlN Stress Relief Layer $-250 \mathrm{~nm}$ \\
\hline GaN Stress Relief Layer $-250 \mathrm{~nm}$ \\
\hline AlN nucleation layer $-40 \mathrm{~mm}$ \\
\hline Silicon $<111>$ Substrate \\
\hline
\end{tabular}

Figure 2.1: Typical GaN HEMT layer structure for growth on silicon $<111>$ substrates

Figure 2.1 shows a typical GaN HEMT layer stack, including the location of a 2DEG. MOCVD (Metal-Organic Chemical Vapour Deposition) or MBE (Molecular Beam Epitaxy) techniques are used to grow these layers on silicon $<111>$ substrates. The band diagram for this structure is shown in Figure 2.2.

\subsubsection{Growth and Process Description}

A thin aluminum-nitride nucleation layer is used to initiate the growth of GaN on the silicon substrate. This nucleation layer is also used for stress relief between the GaN stack and the silicon substrate. 


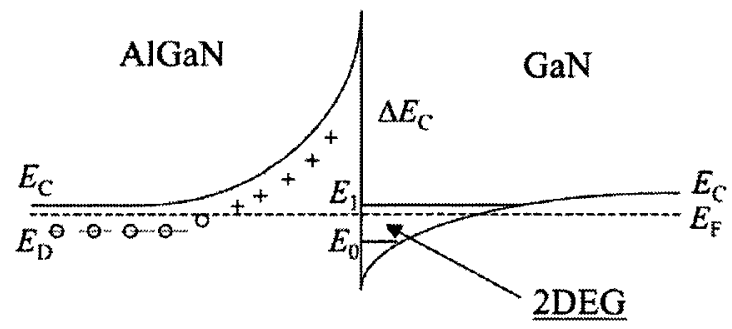

Figure 2.2: Conduction band diagram of GaN HEMT [25]

The next step in the growth process is to grow a $250 \mathrm{~nm}$ thick GaN layer, followed by a $250 \mathrm{~nm}$ thick AlN layer. This is done in order to provide a stress relief layer before the thick GaN buffer is grown. Upon relaxation after growth, micro-cracks typically form in this stress relief layer, which alleviate the stress in the GaN buffer and channel regions.

The GaN buffer layer is typically in the order of $1 \mu \mathrm{m}$ to $3 \mu \mathrm{m}$ thick to minimize the number of dislocations at the crystal surface. As this layer is made thicker, the quality (reduced dislocation density) improves at the cost of higher stress. This layer is typically desired to be semi-insulating such that the devices are electrically isolated. NRC uses a carbon-doping technique to improve the insulating properties of this layer [26]. Iron doping is also commonly used to improve the insulating properties of the GaN layer [27]. Often, when grown by MBE, this layer is naturally insulating.

A thin AlGaN barrier layer, typically in the order of $10-100 \mathrm{~nm}$, is then grown to produce an electron-confining heterojunction that forms the 2-DEG. A piezoelectric polarization component is caused by the tensile strain resulting from the growth of AlGaN on GaN. This adds to the spontaneous polarization that already exists in the GaN. The addition of both of these polarization components gives a positive charge at the interface, which in turn results in the formation of an electron gas right below the interface. The aluminum concentration and thickness of the AlGaN determine the charge density of the 2DEG [28]. Additionally, ultra-thin pure AlN spacer layers 
can be used in the cap to increase confinement.

Extremely thin $(10 \AA)$ GaN cap layers have also been deployed on the top of the structure in order to increase the Schottky barrier height of the gate contact [29]. In addition, the GaN cap layer improves reliability by preventing the oxidation of aluminum in the AlGaN barrier layer. The growth process used for this work is presented in more detail in Section 4.2.1.

Thin-film metal stacks are used to form ohmic contacts to both the source and drain. Typical strategies include the use of $\mathrm{Ti} / \mathrm{Al} / \mathrm{Ti} / \mathrm{Au}$ or $\mathrm{Ti} / \mathrm{Al} / \mathrm{Ni} / \mathrm{Au}$ stacks, which are subsequently rapid thermal annealed at approximately $850^{\circ} \mathrm{C}$. Ohmic contacts to AlGaN/GaN are challenging because of the large bandgap, and hence, multilayer alloys are needed to provide a low work-function contact and reduce the potential barrier to the metal. Section 5.4 provides a detailed explanation of ohmic contact operation and formation. Schottky gates are formed using Pt/Au or Ni/Au stacks.

\subsubsection{HEMT Operation}

Unlike conventional silicon MOSFETs, the HEMT is in the "ON" state when no voltage is applied to the gate. If a potential is placed across the drain to the source, current will flow. Once a negative potential is placed on the gate, the concentration of carriers in the 2-DEG is reduced. The negative potential that fully turns off the channel is known as the pinch-off voltage. The high mobility of the 2-DEG layer allows for high transconductance, and high frequency operation. Additionally, the large bandgap $(3.4 \mathrm{eV})$ results in very high breakdown voltage and higher temperature operation as electrons from the valence band need much more energy to be excited to the conduction band. 


\subsection{GaN Innovations}

Initial development of GaN HEMT devices was plagued with an effect termed "current collapse," which refers to a sagging of transistor IV curves after sustained operation with the channel pinched off and large drain bias. The source of this collapse was attributed to surface states trapping charge on the drain side of the gate, causing a virtual-gate effect on the channel [30]. The primary technique shown to mitigate the effect of these traps is to deposit a SiN passivation layer over the entire device [9]. The SiN passivation provides atomic hydrogen to the surface, which satisfies the dangling chemical bonds forming the traps [9]. Modern devices no longer experience current collapse as the SiN passivation is universally employed. Figure 2.3 shows the effect of the SiN passivation on a sample from NRC.
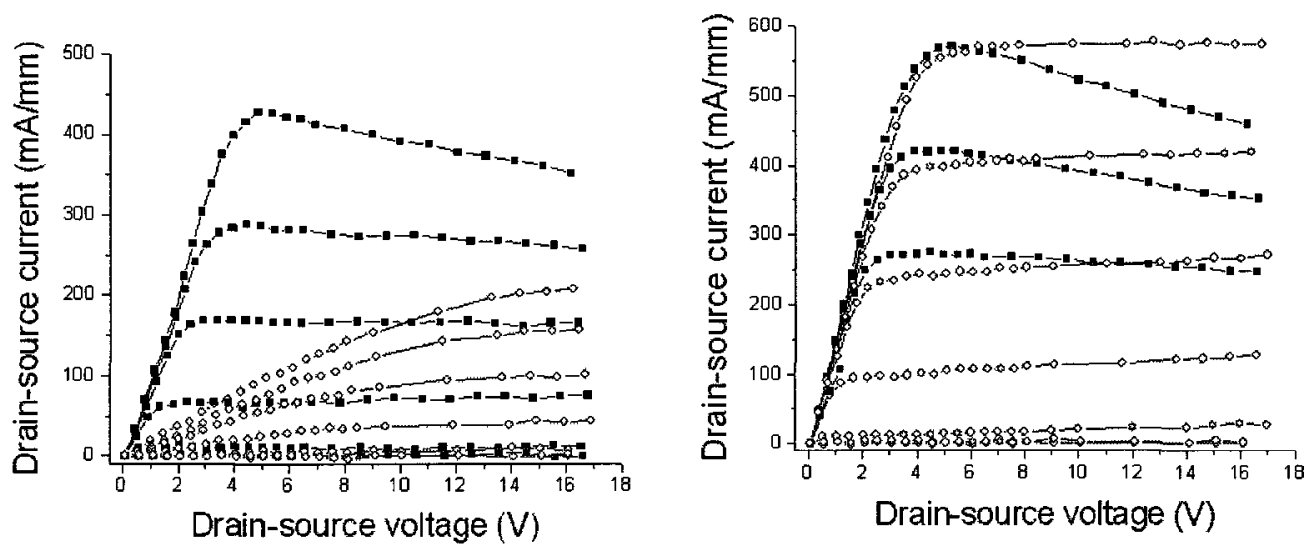

Figure 2.3: Non-passivated (left) and passivated (right) device IV curves showing mitigation of current collapse using pulsed measurements on sapphire substrates (pulsed measurement represented by squares, continuous sweep represented by circles).

Another innovation that has improved the performance of $\mathrm{GaN}$ devices is the introduction of a field plate. A field plate is an extension of the gate over the drain side of the device used to reduce the peak electric field that forms near the drain-side edge of the gate [9]. This has been shown to significantly increase the breakdown 
field of the device and is almost always used for high-power devices [31].

\subsection{Low Temperature MOS processing}

The thermal budget of the MOS processing is the total amount of thermal stress that the wafer experiences. Minimizing the thermal budget is a primary obstacle in this work as will be shown in Section 3.3. Hence, in order for this technology to be commercially viable, there must be a method to fabricate state-of-the-art MOS devices while keeping the thermal budget minimized.

Historically, high temperature steps in MOS processes have been needed for the gate oxidation, as well as for annealing damage and activating dopants following implants used to form source and drain junctions. The need for increased transistor scaling and geometry minimization has forced the industry to move from a silicondioxide based gate dielectric to a high-k material, such as a $\mathrm{HfO}_{2}, \mathrm{HfSiO}$, and $\mathrm{HfSiON}$ [32]. This in turn has greatly reduced the thermal budget allocated to this step, as these new compounds are typically deposited using atomic layer deposition, which is performed at lower temperatures $\left(200^{\circ} \mathrm{C}-400^{\circ} \mathrm{C}\right)$ compared to silicon oxidation.

Thermal budget minimization is also needed to reduce diffusion of implanted junctions in order to avoid short channel effects in small geometry FETs [33]. Two techniques that have been recently developed to successfully anneal and activate dopants in junctions, but not cause unwanted diffusion, are laser annealing and flash lamp annealing [34] [33]. Flash lamp, or spike annealing, involves heating the sample to elevated temperatures (between $950^{\circ} \mathrm{C}$ and $1200^{\circ} \mathrm{C}$ ) for between 1 and 10 seconds. This technique has been shown to effectively form shallow junctions, allowing for technologies smaller than $50 \mathrm{~nm}$, which cannot be accomplished using conventional RTA techniques. Laser annealing is an alternative to spike or flash lamp annealing that can anneal for bursts in the millisecond range, allowing for the formation of 
shallower junctions [34]. This technique has been used to push MOS technology to gate lengths smaller than $30 \mathrm{~nm}$.

\subsubsection{Low Temperature Oxidation of Silicon}

The growth of a high quality gate oxide is the most important step in fabricating silicon MOS devices. Because of the thermal budget constraints of this work, it is important to examine the minimum temperature and conditions that can be used for this step, without sacrificing device quality. Traditionally, thermal gate oxides have been grown at temperatures greater than $1000^{\circ} \mathrm{C}$ in order to produce a high quality film which has low leakage and high dielectric strength [35]. EerNisse et al. have also shown a significant stress reduction in $\mathrm{SiO}_{2}$ layers grown at temperatures above $950^{\circ} \mathrm{C}$, which is the viscous flow point of the film [36].

It has also been shown however, that quality oxide films can be grown in a twostep approach, which consists of a lower temperature deposition, followed by a postoxidation anneal $[37,38]$. Quality oxides have been demonstrated with growth temperatures as low as $800^{\circ} \mathrm{C}$ followed by a post-oxidation anneal at temperatures of $950^{\circ} \mathrm{C}$ and above, which allows for thermal relaxation to take place. The duration of these anneals vary, but the minimum time has been shown to be approximately one minute [37]. Quality is shown to improve with anneal time as well. This twostep approach could be useful in overcoming the thermal budget challenges that this particular project presents.

These processes however, have been developed on the $<100>$ orientation of silicon. There is little literature on low temperature oxidations on the remaining two orientations, which are used in this work. Early research has shown that other orientations of silicon result in oxides that have approximately three times the quantity of interface states as well as increased growth rates [39]. 


\subsection{Towards an Integrated Technology}

Fujikawa et al. have grown a GaN layer on an SOI substrate consisting of a silicon $<100>$ substrate, a buried oxide, and a thin silicon $<111>$ top layer [40]. This process enables the recovery of a smooth silicon surface, necessary for potential MOS integration. The oxide under the top-level silicon layer acts to protect the underlying surface. An advantage of this technique is the ability to grow on a silicon $<111>$-oriented surface, which is bonded to a $<100>$-oriented substrate: the preferred orientation for MOS fabrication. HEMT layers have yet to be grown in this manner. This technique may be limited by the availability of custom SOI wafers, as it requires a highly specialized order due to the different wafer orientations and small diameter. However, this could still be more cost effective than the use of $\mathrm{SiC}$ substrates.

Shichijo et al. have integrated MOS with GaAs MESFET devices [41]. This is the first example of integrating MOS devices with III-V technology. An advantage of integrating GaAs with MOS as opposed to GaN is the ability to grow the GaAs selectively in a silicon trench at reasonably low temperatures. GaAs can be grown in the $450^{\circ} \mathrm{C}$ range, whereas $\mathrm{GaN}$ epitaxy reaches a temperature range from at least $800^{\circ} \mathrm{C}$ to more than $1000^{\circ} \mathrm{C}$ for several hours. This difference in growth temperature allows for a much more relaxed thermal budget when designing the MOS process, as the effect of the GaAs growth on the doping profile was negligible for the technology that was used at the time. Although a MOS-first approach would be better, current sub-micron technology would not be able to withstand the GaN HEMT growth step, and hence an approach discussed in Section 3.2 has been taken for this work.

An example of a combined GaN/CMOS hybrid chip has also been implemented by Matsunaga et al. [42]. Similar to the circuit to be implemented in this work, a dynamic gate supply voltage circuit in CMOS is used to improve the linearity and efficiency of a class-B GaN power amplifier. It is evident that the ability to integrate 
both CMOS and GaN transistors on the same chip would be highly beneficial to this technology.

Chung et al. have recently accomplished a similar integration to that of this work by using a very different technique [43]. This is based on a wafer-bonding scheme that allows for the use of $\langle 100\rangle$ oriented silicon for the MOS devices, which is currently the preferred orientation for this technology. This involves attaching two wafers together using an adhesion layer and the application of high pressure. The disadvantage to using a wafer bonding technique, however, is two-fold. Firstly, the maximum substrate size that can be bonded is very limited, currently set at one square inch. If this technology is to be economically feasible for mass production, the substrate size must be increased substantially. Secondly, the long-term reliability of such a technique has not been well-studied. This is especially important for GaNbased technology; one of its primary strengths is the ability to operate in harsh environments.

The present work, which is based on an epitaxial process that monolithically integrates both MOS and GaN technology, is better suited for commercialization, as there are no obstacles to limit scaling to much larger substrates. In addition, there is less concern about long-term reliability due to the nature of the process. The MOS devices are not based on a $<100>$ oriented substrate, but it appears that $<110>$ oriented silicon could house devices with comparable performance as research continues in this field. 


\section{Chapter 3}

\section{Integration Strategy and Considerations}

In this chapter the primary challenges involved in the monolithic integration of GaN HEMT and MOS technologies are discussed at a strategic level without delving into a high level of detail about the process. There are multiple ways to approach the integration problem, but only a few that are able to overcome the processing obstacles are presented. It is imperative that a process be designed in which the performance of both GaN and MOS devices is not compromised. Growth, fabrication, and thermal issues are all taken into account. The strategy used to approach the integration problem in this work is then given, which is based on the qualitative observations presented here.

\subsection{Growth Considerations}

The first major challenge that must be considered is finding a method by which to obtain regions of atomically smooth silicon alongside active GaN layers on the same substrate. It is important that the silicon surface be of the utmost quality for a good $\mathrm{Si}-\mathrm{SiO}_{2}$ interface for the MOS devices. The simplest way of attempting this is to simply etch away regions of GaN on a full planar growth to expose the silicon surface. However, this does not result in an atomically smooth surface as shown in Section 4.1. 
This technique could be feasible by growing on a silicon-on-insulator (SOI) wafer, and then wet etching away the silicon film and buried oxide after removing the $\mathrm{GaN}$, but the availability of $\langle 111\rangle$ or $\langle 110\rangle$ oriented SOI is low. However, the SOI idea is attractive if the $<100>$ orientation is specifically needed for the MOS devices, as a $<111>$ on $<100>$ wafer can be prepared.

An alternative approach is to attempt selective growth, in which growth occurs only in specific areas of the silicon wafer, to preserve regions for MOS integration. This technique requires preparation of the silicon sample before growth, which may compromise the smooth silicon surface or introduce unwanted materials into the growth chamber. Although the orientation of the silicon may not be ideal for state-of-the-art CMOS, this approach may give an advantage with respect to preserving an atomically smooth surface for growth.

\subsection{Processing Background}

Figure 3.1 shows the main steps for both a CMOS and GaN process flow that can be performed with current technology available at Carleton University and NRC, respectively.

There are a few observations from these flows that have important consequences for integrating them. Firstly, all MOS process steps except for the contact formation and metallization require temperatures of $900^{\circ} \mathrm{C}$ and above. Typically, the gate and field oxidations require over $1000^{\circ} \mathrm{C}$.

The GaN process is far simpler than the MOS, as it requires fewer steps and no long high-temperature exposure. The only high-temperature steps that are required are to anneal the ohmic contacts, which is typically done at $850-900^{\circ} \mathrm{C}$. The only step of the GaN process that is able to withstand any of the MOS processing would be the mesa etch. After metal is deposited for the ohmic contacts, no more long-term 




Figure 3.1: Primary steps for both CMOS and GaN processes

high temperature steps can be performed on the sample. The only steps of the MOS process that cannot withstand high temperatures are the back end metallization steps. This suggests that the order in which the steps are performed is not interchangeable.

A similar module in these two flows is the final back-end process, which includes the final metallization steps. Both need contact or via etches through a dielectric followed by a metal fill. Aluminum is currently used for the MOS process, and gold is used for the GaN process as it has higher resistance to electromigration. Unfortunately, gold is not compatible with the CMOS process. Some changes will need to 
be made to accommodate this (see Section 5.3). Connecting both types of transistors is an important part of the back-end process. A common metal scheme must be found that satisfies the performance requirements of the devices. Additionally, MetalInsulator-Metal (MiM) caps and spiral conductors can be included in this stage of the process.

Another factor that must be accounted for is the step height expected between the silicon regions and the GaN stack, which is typically around $2 \mu \mathrm{m}$. This will depend on the approach used to obtain a wafer with areas for MOS and GaN devices.

Combining the processing steps also means that some thought must go into protecting the MOS or GaN regions of the chip while processing is performed on the other regions. Ideally these steps can be built into the process without much added work. A preferred solution is to use existing steps in these flows, such as thick dielectric depositions, to double as protective layers. Extra photolithography steps that protect MOS or GaN regions of the wafer while processing is performed on the other region are also needed.

\subsection{Thermal Budget Considerations}

From the above discussion it is clear that a major concern in integrating MOS with GaN devices is the ability of the GaN HEMT layers to withstand the thermal budget of the MOS processing. Preliminary experiments run with a standard HEMT growth on an $\mathrm{Si}<111>$ substrate revealed how difficult this issue would be. Pieces of the same wafer were annealed at different temperatures for 2 hours in a nitrogen environment in order to mimic the thermal stress of the MOS process. Before annealing, the samples were passivated with a thick PECVD oxide to cap the GaN and mitigate GaN decomposition. Figure 3.2 shows the resulting SEM and optical microscope images when a sample was annealed at $1000^{\circ} \mathrm{C}$ for 2 hours. Decomposition occurs 
along line defects in the $\mathrm{GaN}$ as shown by the optical microscope image in Figure 3.2a. This image shows cracking along the defects as well as sites in which lift-off of the layers has occurred. Dissociation of $\mathrm{GaN}$ is the most likely reason and causes lifting off of different layers. This has been shown to occur at elevated temperatures [44-46]. Ga droplet formation is also shown to occur as per Figure 3.2b, which is a sign of dissociation. $3.2 \mathrm{c}$ and $3.2 \mathrm{~d}$ show the lifting of layers due to $\mathrm{GaN}$ decomposition after the oxide has been removed.

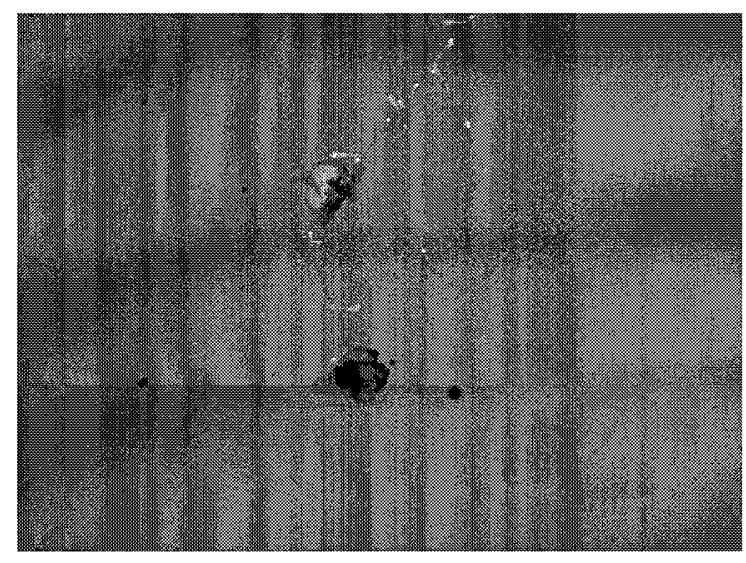

(a) Optical microscope image

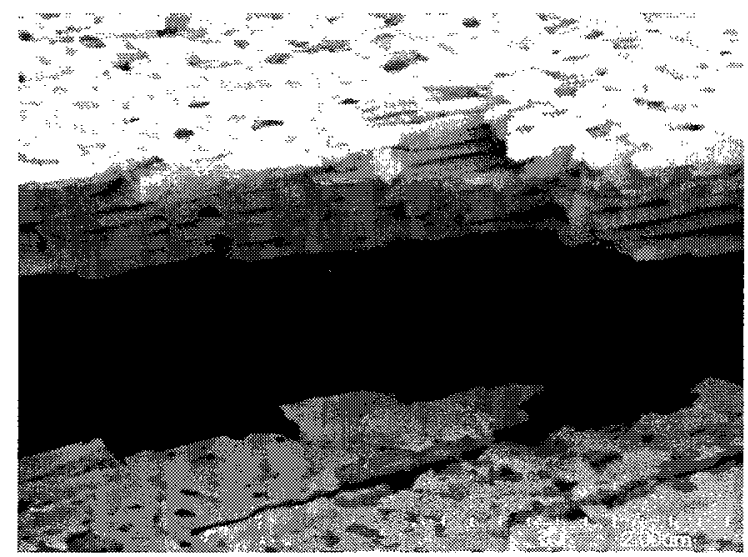

(c) SEM Image - oxide stripped



(b) SEM Image

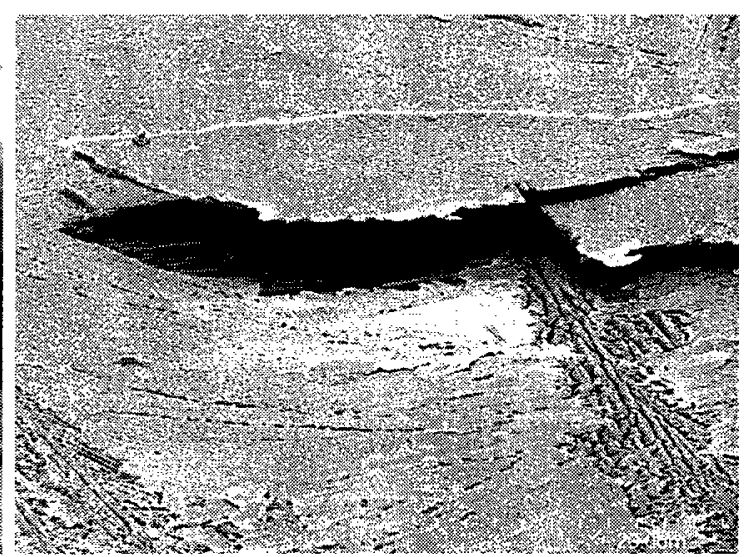

(d) SEM Image - oxide stripped

Figure 3.2: Optical and SEM pictures of sample annealed at $1000^{\circ} \mathrm{C}$ 
extent. Moreover, the extent of decomposition varied between different growth runs. This is consistent with the work of Song et al. [47] as well as Stonertet al. [48], which shows $\mathrm{GaN}$ is mostly stable at $900^{\circ} \mathrm{C}$, after which decomposition occurs. However, a capping layer such as $\mathrm{SiO}_{2}$ should mitigate decomposition as shown in [49] when used to suppress $\mathrm{N}$ dissociation from AlGaN layers during Si implant activation annealing. An AlN capping layer could be considered for this work, but would be very difficult to remove after MOS processing [50]. In addition, reducing the oxide thickness and using a higher quality deposition technique may be needed. The issue of GaN stability must be resolved before MOS fabrication is to take place. It is imperative that a process flow with a limited thermal budget be designed in this case, as this appears to be the major obstacle in successfully accomplishing this integration.

An alternative approach could be to perform the MOS fabrication prior to GaN growth altogether. This would circumvent the issue of GaN decomposition as all the high temperature processing steps could be performed prior to growth. Additionally, the gate oxidation temperature would not be limited, ensuring a high quality layer. This method however, would not be compatible with current state-of-the-art CMOS processing as the thermal budget required for GaN growth would cause unwanted diffusion of shallow junctions, limiting the device geometry and overall performance. There is also the added risk of contamination to the atomically smooth silicon surface required for GaN growth due to the increased number of processing steps performed prior to epitaxy.

\subsection{Thermal Performance Considerations}

In order to integrate these two technologies, thermal effects must be taken into consideration as elevated temperatures hamper device performance. Thermal simulations will be able to predict the behaviour of this chip, allowing for the intelligent layout 
of different components. Previous work in [51] has shown that the thermal gradient resulting from heat generated by the GaN power device has little effect on the chip for separations greater than $20 \mu \mathrm{m}$ from the source, due to the poor thermal conductivity of the silicon substrate. The primary issue discovered by this work is that localized heating of the GaN should be more of an issue, and hence techniques are needed to properly cool the power devices.

\subsection{Final Approach}

Figure 3.3 shows a cross-section of the planned co-integration process. A windowed growth technique has been developed that isolates the silicon surface and the GaN layers on the chip such that both are available for fabrication. This technique is more elegant and cost effective than using a growth on SOI or etching approach, but does have the disadvantage of using $<111>$ or $<110>$ oriented silicon. Chapter 4 gives a more in-depth discussion of this process.

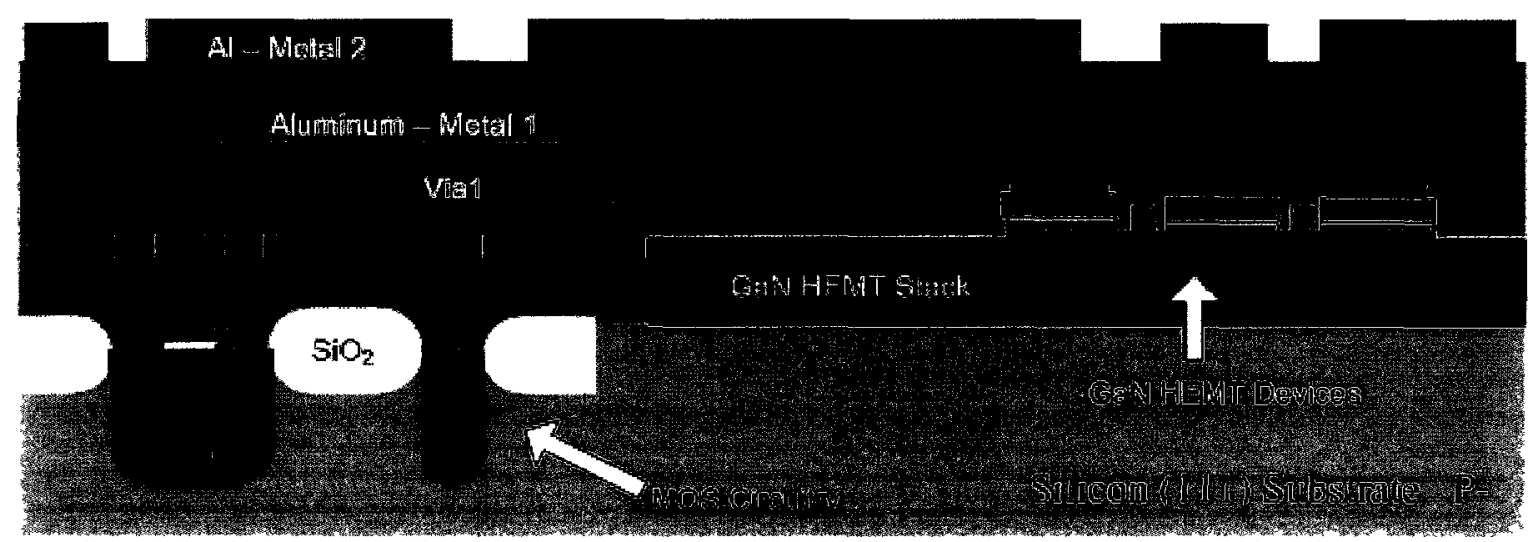

Figure 3.3: Cross section of envisioned co-integration process

The MOS devices are fabricated prior to the GaN devices as the high temperature required for the MOS processing, especially the thermal oxidations, will impact the GaN metallization. It should also be noted that due to the high temperature required for GaN growth, and the high quality of surface required, MBE growth must be 
performed at the beginning of the fabrication process. It is also important to minimize possible contaminants to the silicon surface before growth in order to obtain quality GaN layers.

A joint metallization will be performed in this process in order to take advantage of the similar back-end steps. Unfortunately, gold cannot be used as it forms recombination/generation centers in silicon, resulting in leaky junctions. However, aluminium, which is typically used for MOS processing can also be used for GaN. There is a small performance penalty to pay, as gold has slightly lower resistivity and better electromigration resistance. Overcoming the topological difference on the chip between the GaN and NMOS transistors may pose challenges. The GaN HEMT structure, which is typically around $2 \mu \mathrm{m}$ thick, has a very thin AlGaN cap layer that does not allow for the height of the GaN stack to be modified. Because of this, the topology will not be uniform and a technique such as a dielectric fill or reflow must be used to facilitate uniformity for metallization. Additionally, the difference in height of the MOS and GaN surfaces may pose future challenges for lithography.

A MOS process that does not exceed $950^{\circ} \mathrm{C}$ has been designed. This will mean that growing a good quality gate oxide for the MOS devices could be a challenge. An alternative approach would be to use a deposited gate dielectric as is used in modern CMOS technology as discussed in Section 2.4. In order to be certain about the thermal budget required, annealing tests as described in Section 3.3 were performed on new growths to determine the maximum temperature they can withstand, as this was found to vary between growths.

The GaN processing steps only require a small change to the metallization scheme to avoid gold. A uniform coating to protect this region will be used during the MOS fabrication.

The detailed process design is presented in Chapter 5. 


\section{Chapter 4}

\section{Windowed Epitaxial GaN Growth on}

\section{Silicon}

A primary challenge in integrating MOS devices into a typical substrate used for GaN epitaxy is to recover an atomically smooth silicon substrate for MOS fabrication. This chapter will first summarize the primary obstacles in obtaining such a surface, and present a new method developed in this work that allows for selected areas of the silicon substrate to be preserved for MOS fabrication. The electrical and mechanical properties of the resulting wafers are then discussed.

\subsection{Primary Challenges}

In the case of full wafer planar growth as described in Section 2.2.1, recovering a smooth silicon surface by etching the $\mathrm{AlGaN} / \mathrm{GaN}$ heterostructure layer is complicated. Buried cracks in the buffer layer stack usually used to compensate the strain [52] can be translated into the silicon surface. An additional concern is the possibility of a change in silicon morphology and purity due to the Si/AIN interface used at the early stage of the growth. Figure 4.1a illustrates the pattern of buried cracks transferred into the silicon surface during Inductively Coupled Plasma (ICP) 
etching to remove the AlGaN/GaN stack. Focussed Ion Beam (FIB) imagıng shows that these cracks originate between the AlN and GaN stress-relief layers (Figure 4.1b) The poor silicon morphology would interfere with MOS fabrication.

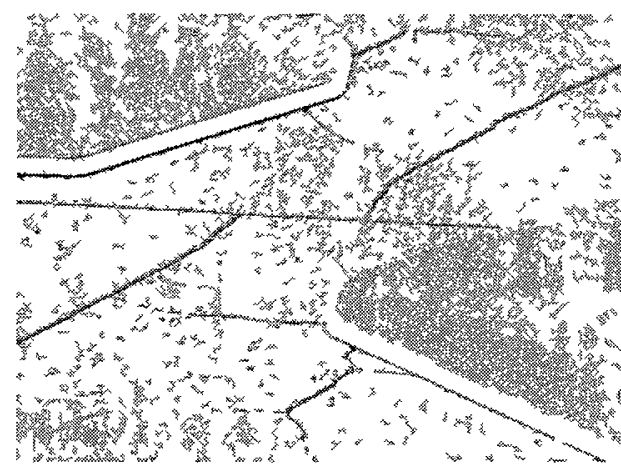

(a) Etched GaN

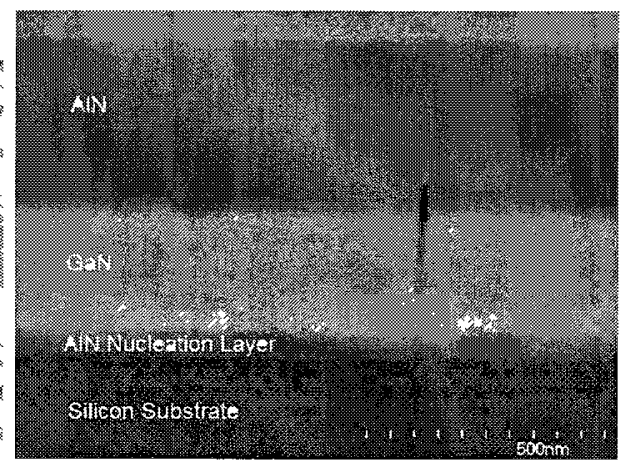

(b) FIB Section

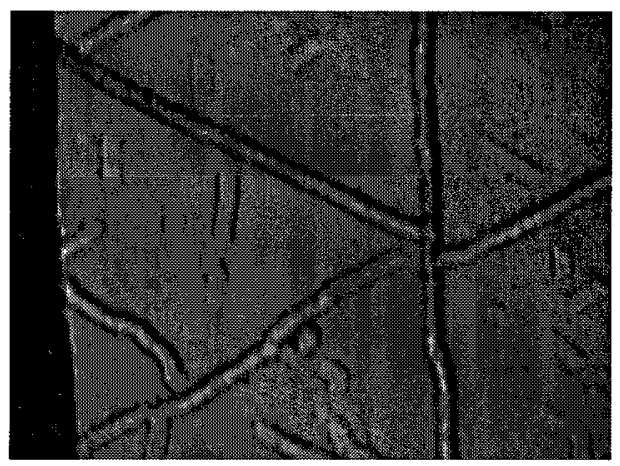

(c) Smoothing

Figure 4.1: a SEM image of AlGaN/GaN heterostructure ICP etched down to the silicon surface. Note the poor quality of the silicon surface which includes both cracks and surface pits. b. FIB Section of wafer showing cracks originating between the stress relief layers. c. Optical microscope image of an attempted smoothing of microcracks using TMAH.

Attempting to smooth the silicon surface by wet etchung using TMAH $[53,54]$ and $\mathrm{NH}_{4} \mathrm{~F}[55-57]$ did not improve the silicon morphology. The microcracks etched into the silicon surface expose new etch planes to the crystallographıc etch, which causes wider channels to form in the surface. This is demonstrated in Figure 4.1c.

Growth using maskless mesas on sllicon $<111>$ substrates using patterned AlN 
nucleation layers is another option in pursuing a co-integrated technology [58]. However, there is no way to protect the exposed silicon growth from contamination during growth, making it challenging to incorporate MOS devices.

\subsection{Windowed Growth}

A new windowed growth technique has been developed which preserves the smooth surface of the silicon substrate during MBE growth of the GaN heterostructure [59]. Rather than attempting to recover the silicon surface after growth, this technique, known as differential epitaxy, uses a dielectric passivation to protect the surface before growth by ammonia MBE [60]. A thick dielectric coating of silicon dioxide followed by a thin amorphous silicon layer is deposited uniformly over the entire substrate. A combination of dry and wet etching is used to open up windows in the dielectric, exposing the silicon surface for epitaxy. The role of the amorphous silicon is to allow for nucleation on the surface, that in turn can be lifted off by simply wet-etching the $\mathrm{SiO}_{2}$. In addition, the amorphous silicon prevents exposure of the $\mathrm{SiO}_{2}$ layer during growth, as oxygen contamination in the GaN buffer layer may be an issue.

Figure 4.2 summarizes the two techniques used to isolate a smooth silicon surface. As discussed, etching and smoothing is not a viable option due to the microcracking in the GaN stress-relief layers.

Figure 4.3 shows the wafer before MBE growth. The silicon surface for epitaxy appears very smooth, which should ensure a good quality GaN HEMT. The thick silicon dioxide is used for lift-off.

Figure 4.4 shows the silicon surface after windowed growth. Note the smooth appearance in stark contrast of that to Figure 4.1, in which dry etching was used after growth of the AlGaN/GaN heterostructure. The development of this technique has been the major enabling step that allows for MOS integration with $\mathrm{AlGaN} / \mathrm{GaN}$ 


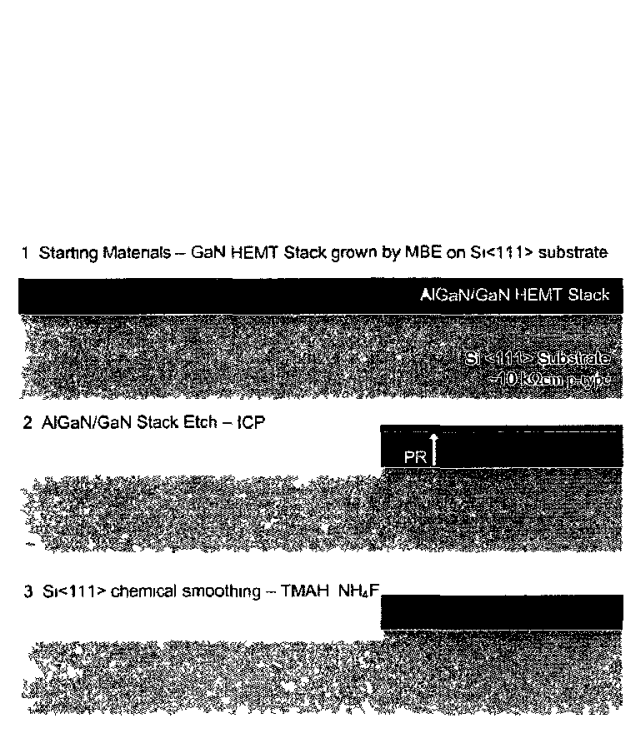

(a) Etch and Smooth

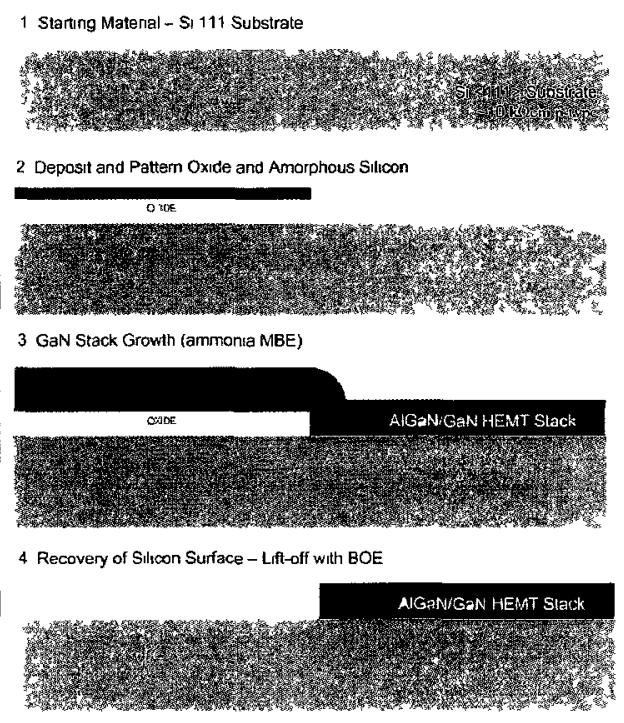

(b) Windowed Growth

Figure 4.2: Comparison of growth techniques

HEMTs.

\subsubsection{Detailed Growth Process}

\section{Dielectric Coating}

The original windowed growth technique employed a $1.8 \mu \mathrm{m}$ thick dielectric coating of silicon dioxide, followed by a $0.1 \mu \mathrm{m}$ thick amorphous silicon layer deposited uniformly over the entire substrate. The oxide layer was deposited using a model 700 Plasmatherm PECVD system, with an RF power of $40 \mathrm{~W}$ and temperature of $350{ }^{\circ} \mathrm{C}$. The a-Si layer was deposited using a custom magnetron sputtering system with a boron-doped silicon target, using a power of $100 \mathrm{~W}$ at room temperature. The custom system consists of guns from US Inc. (US Gun II model), an Advanced Energy MDX-1K power supply, and a CTI model CT8 cryo pump.

Due to poor adhesion of a-Si to the oxide layer in some samples, the a-Si step 


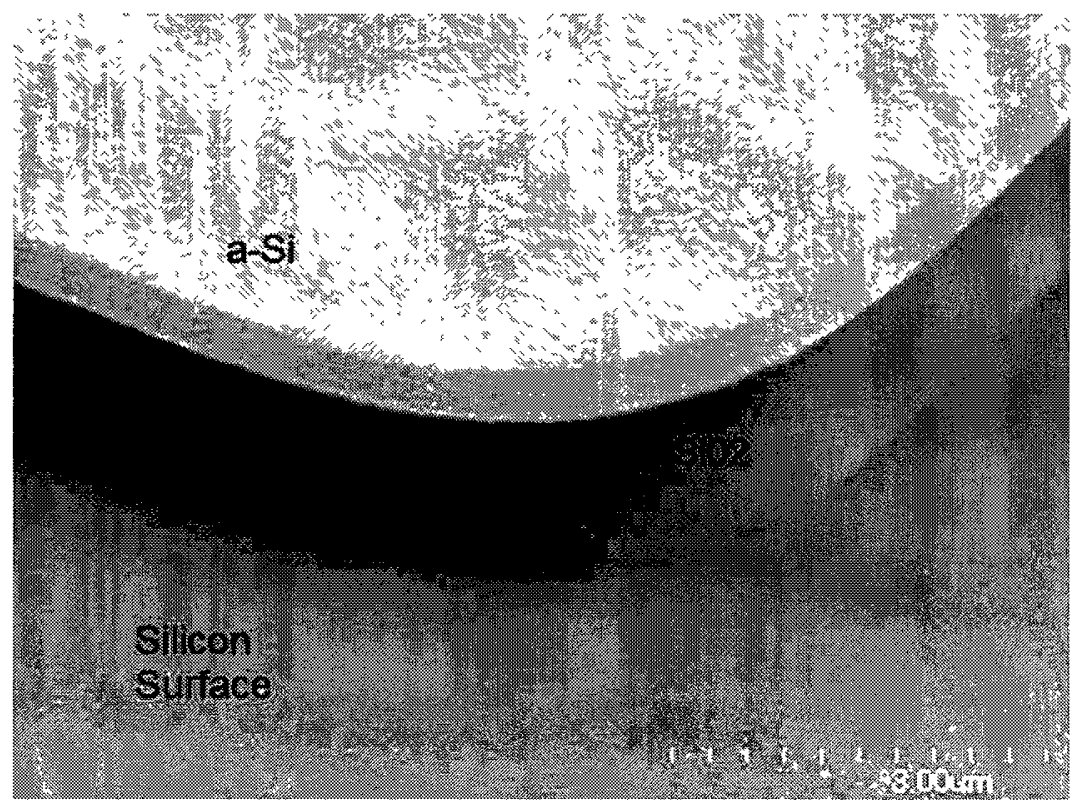

Figure 4.3: Wafer before MBE growth step. Note smooth silicon surface available for epitaxy.

was changed from room temperature sputtering to a $560^{\circ} \mathrm{C}$ LPCVD a-Si deposition, followed by a 1 hour anneal to crystallize the layer at $560^{\circ} \mathrm{C}$. Additionally it was determined that a $1 \mu \mathrm{m}$ oxide layer is adequate for lift-off rather than $1.8 \mu \mathrm{m}$, reducing the probability of oxygen outgassing.

In both cases a combination of dry and wet etching are used to open windows in the dielectric, exposing the silicon surface for nitride epitaxy such that there is no etch damage. First, an ICP etch using a $C_{4} F_{8}$ and $O_{2}$ chemistry is used to etch until approximately $100 \mathrm{~nm}$ of $\mathrm{SiO}_{2}$ is left on the silicon areas. Next, a buffered oxide etch (BOE) with surfactant is used to etch the remaining $\mathrm{SiO}_{2}$ down to the silicon surface. This method results in a slight undercut of the protective dielectric, but avoids plasma etch damage to the silicon surface, which may result in a poor growth quality. The slight undercut is beneficial in facilitating GaN liftoff. 


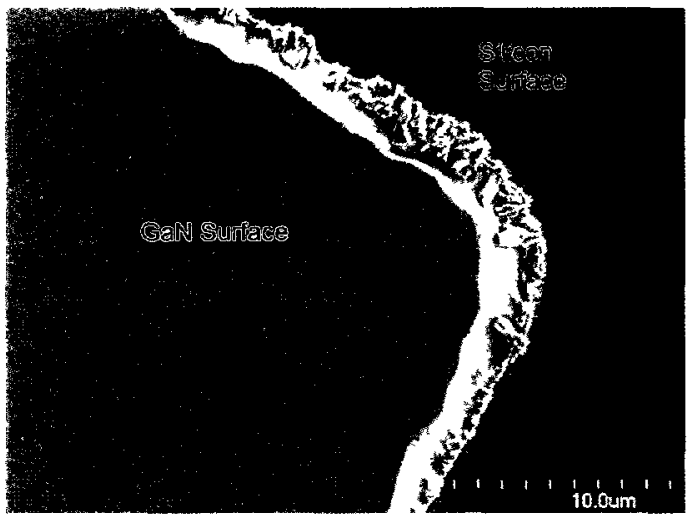

(a) SEM image of GaN and Silicon on same substrate.

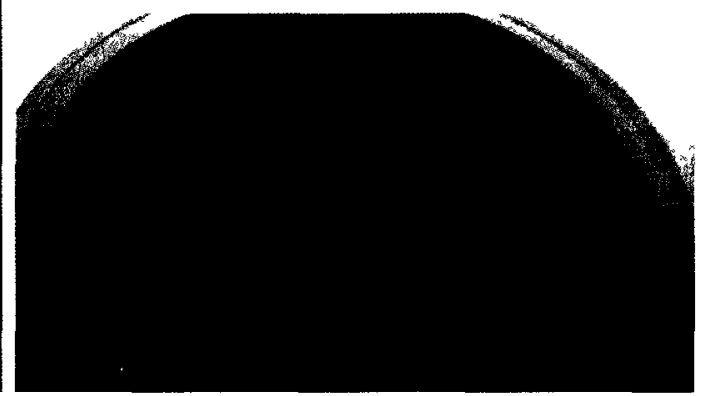

(b) Full wafer showing Silicon Windows.

Figure 4.4: Windowed growth images

\section{Epitaxial Growth}

Samples are grown by MBE using ammonia as the nitrogen precursor. Initially, carbon-doped material was used in order to increase the resistivity of the buffer layer, however in order to simplify the growth, unintentionally-doped GaN layers were used instead. A description of both techniques is given.

After window fabrication, but immediately prior to the growth, 2" high resistivity silicon $<111>$ substrates are chemically cleaned and then the native oxide is removed using an $\mathrm{HF} / \mathrm{H}_{2} \mathrm{O}$ solution. This etch time is short so little of the $\mathrm{SiO}_{2}$ mask is removed. Once loaded in the growth reactor, substrates are heated to about $700^{\circ} \mathrm{C}$ to desorb the hydrogen present at the surface, and a $7 \times 7$ surface reconstruction is observed using RHEED (Reflection High-Energy Electron Diffraction). A growth process identical to the one used in reference [61] is applied. It consists of first growing a $40 \mathrm{~nm}$ AlN layer at a typical growth rate of $0.1 \mathrm{~mm} / \mathrm{h}$ while the growth temperature is increased from $650^{\circ} \mathrm{C}$ to $920^{\circ} \mathrm{C}$. Then GaN and AlN stress relief layers, both about $250 \mathrm{~nm}$ thick, are grown at $800^{\circ} \mathrm{C}$ and $920^{\circ} \mathrm{C}$, respectively. This technique has been shown to absorb the tensile stress due to the high lattice and thermal expansion coefficient mismatch of the silicon substrate relative to the GaN layers [61]. Next, a 
thick $1.4 \mu \mathrm{m}$ C-doped GaN buffer layer is used to improve electrical insulation [26]. The structure is completed by growing the active layer, which consists of a $200 \mathrm{~nm}$ undoped GaN channel, a thin AlN spacer, and an AlGaN barrier (20 nm at 30\%) covered by a $2 \mathrm{~nm}$ GaN cap layer. The ammonia flow rate is maintained constant over the whole growth process at $200 \mathrm{sccm}$.

Unintentionally doped material (i.e. without carbon doping) follows a similar process described above except that the methane gas used to dope the GaN buffer layer with carbon is not used. The $0.2 \mu \mathrm{m}$ undoped GaN layer is therefore not included since the entire buffer layer is unintentionally doped. Although no extra dopant is added, defects in the growth and background contamination make the buffer layer semi-insulating. In addition, no GaN cap layer is used. Figure 4.5 shows cross sections of both structures.

\begin{tabular}{|c|}
\hline $\begin{array}{l}\text { AIGaN Barrer }(30 \% \text { Al) }-20 \mathrm{~nm} \\
\text { AIN spacer }-15 \mathrm{~nm} \\
\text { GaN Channel }-200 \mathrm{~nm}\end{array}$ \\
\hline GaN Buffer (C-doped) 14 um \\
\hline AIN Stress Relief Layer $\sim 220 \mathrm{~nm}$ \\
\hline $\begin{array}{l}\text { GaN Stress Relief Layer } \sim 220 \mathrm{~nm} \\
\text { AIN nucleation layer } \sim 40 \mathrm{~nm}\end{array}$ \\
\hline Silicon $\langle 111\rangle$ Substrate \\
\hline
\end{tabular}

(a) Carbon-doped growth layer structure.

\begin{tabular}{|c|}
\hline AlGaN Bamer $(30 \%$ An) $-20 \mathrm{~nm}$ \\
\hline AlN spacar $-75 \mathrm{nmm}$ \\
GaN Buffer/Channel (undoped) $\sim 16 \mathrm{um}$ \\
\hline AIN Stress Relief Layer $\sim 220 \mathrm{~nm}$ \\
\hline GaN Stress Relief Layer $\sim 220 \mathrm{~nm}$ \\
\hline AlN nucleation layer $\sim 40 \mathrm{~nm}$ \\
\hline Silicon $<111>$ Substrate \\
\hline
\end{tabular}

(b) Unintentionally-doped growth layer structure.

Figure 4.5: GaN layer structure for growth on silicon $<111>$ substrates 


\begin{tabular}{|c|c|c|}
\hline Property & C-Doped Growth & Unintentionally-doped Growth \\
\hline Sheet Resistance & $442 \mathrm{Ohms} / \mathrm{sq}$ (hall bars) & 445 Ohms/sq (corbino) \\
Mobility & $1.37 \times 10^{3} \mathrm{~cm}^{2} / \mathrm{Vs}$ & $917 \mathrm{~cm}^{2} / \mathrm{Vs}$ \\
Sheet Carrier Concentration & $1.03 \times 10^{13} \mathrm{~cm}^{-2}$ & $1.07 \times 10^{13} \mathrm{~cm}^{-2}$ \\
T-max before decomposition & $<950^{\circ} \mathrm{C}$ & $>950^{\circ} \mathrm{C}$ \\
Forward Current Achieved & $0.60 \mathrm{~A} / \mathrm{mm}$ & $0.81 \mathrm{~A} / \mathrm{mm}$ \\
Pinch-off Voltage & $-5.6 \mathrm{~V}$ & $-3.3 \mathrm{~V}$ \\
Leakage Current (Vgs=Vds=-10 V) & $1 \times 10^{-2} \mathrm{~A} / \mathrm{mm}$ & $1 \times 10^{-2} \mathrm{~A} / \mathrm{mm}$ \\
\hline
\end{tabular}

Table 4.1: Comparison of the properties of the best C-doped and non C-doped growth wafers

\subsubsection{Windowed Growth Wafer Properties}

The properties of the gallium nitride layers produced by this technique must be comparable to those for full planar growths in order for this work to be of value. The electrical properties of the windowed growth layers have been characterized using hall measurment and capacitance-voltage (CV) analysis. SIMS has also been used to examine the oxygen concentration in the buffer layers.

\section{Hall Measurements}

For the initial C-doped samples Hall bar structures were formed by first dry etching the GaN using a chemically assisted ion-beam etch (CAIBE) to form isolated mesas. This was followed by a $\mathrm{Ti} / \mathrm{Al} / \mathrm{Ti} / \mathrm{Au}$ ohmic metallization. For the non C-doped samples, cylindrical symmetry TLM structures (Corbinos) were used to determine sheet resistance, as well as the ohmic contact resistance [62]. A Hall probe measurement on the full wafer was utilized to determine the mobility and carrier density. These properties are summarized in Table 4.1.

The sheet resistance and carrier density for the unintentionally-doped samples were very close to that of the C-doped samples, but the mobility was slightly lower. 
The results for both types of growth are comparable to that of a full planar growth on silicon, suggesting that this technique does not degrade the quality of the GaN layers. These values do vary for each growth due to the variability of growth parameters that are used during the epitaxial process.

\section{CV measurement}

The free carrier density deduced from capacitance-voltage (CV) measurements is plotted in Figure 4.6 for the C-doped samples. It should be noted that these measurements were performed on a different growth wafer than that of the Hall results. The residual carrier concentration in the buffer layer is around $5 \times 10^{14} \mathrm{~cm}^{-3}$. This value spikes in the stress-relief layer, most likely due to impurities as a result of the sacrificial dielectric layer. A pinch-off voltage of around $-4.7 \mathrm{~V}$ is also measured.

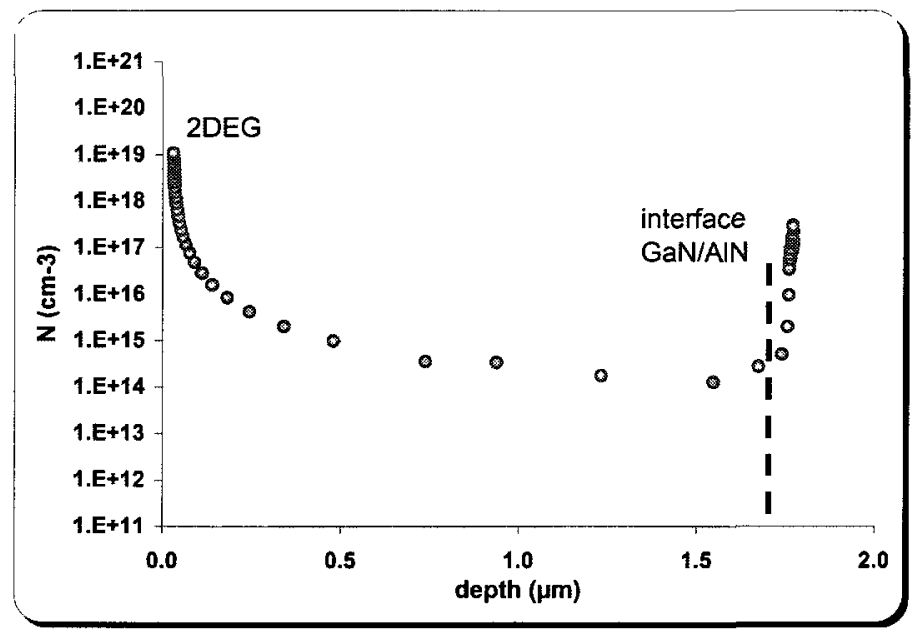

Figure 4.6: CV carrier concentration profile in the 2DEG and GaN buffer

The measurements were performed by CNRS-CHREA in France using a MDC probe station in conjunction with an Agilent 4284 LCR meter. The measurement was performed at a frequency of $10 \mathrm{KHz}$. 


\section{Other Electrical and Physical Properties}

The primary electrical properties determined after fabrication of test devices are summarized in Table 4.1. These will be further investigated in Chapter 7 .

An important parameter to note for this table is that the non-carbon doped material does have similar leakage current to the $\mathrm{C}$-doped material at a reasonable negative bias of $-10 \mathrm{~V}$ on the gate. However, as the gate voltage becomes more negative, the leakage current in the $\mathrm{C}$-doped material remains relatively constant, while the non C-doped material becomes more leaky. This result is presented in Section 7.2.

The thermal stability of the material is also of interest. The C-doped material was found to decompose at a temperature of $900^{\circ} \mathrm{C}$, whereas the unintentionallydoped growth is stable at this temperature, which will allow for a higher temperature thermal oxide growth. This is essential for making high quality MOS devices based on a thermal oxidation process. It should be noted that C-doping may not be a direct cause of thermal instability, but just a result of the C-doped growth not being optimized in this work.

\section{SIMS Analysis of C-doped samples}

Secondary ion mass spectroscopy (SIMS) is used to determine the effects of the exposed oxide on the HEMT stack, as oxygen contamination may be an issue. Outgassing of the oxygen in the $\mathrm{SiO}_{2}$ sacrificial layer poses a concern during growth. Oxygen impurities are not desired in the GaN buffer layer, as they cause unintentional n-type doping, which reduces the insulating properties of the layer [63-66].

SIMS is used to analyze the oxygen incorporation in the GaN HEMT layers for both a full wafer epitaxial growth and the windowed growth. Figure 4.7 shows the SIMS oxygen and carbon concentration profiles for both the standard and windowed growth samples. 


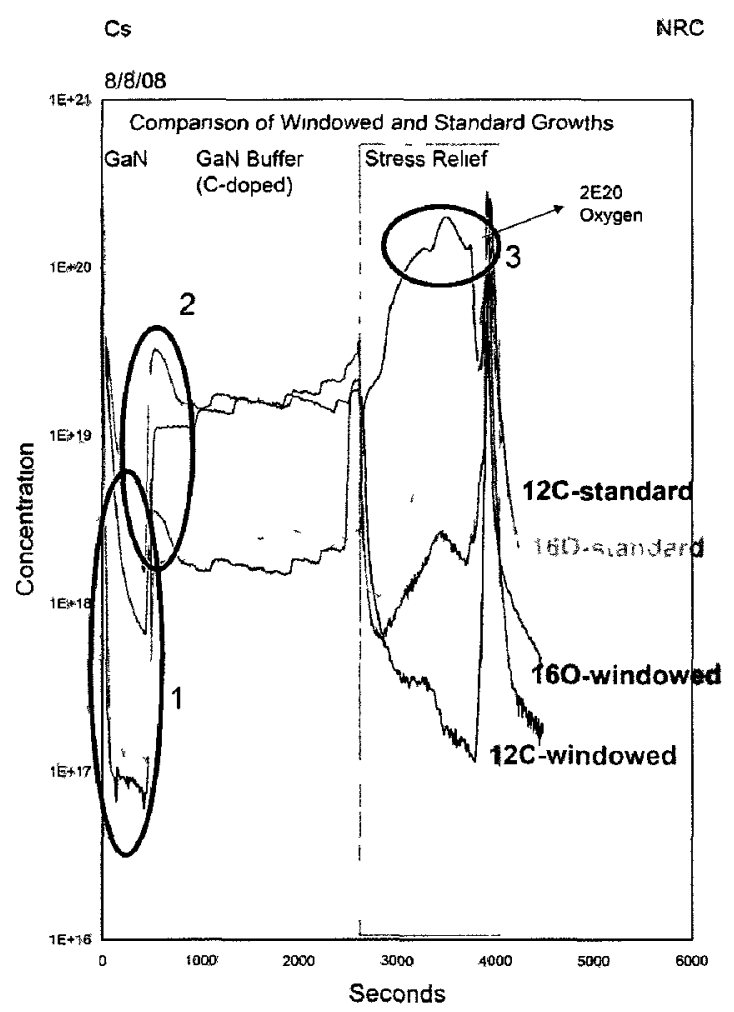

Figure 4.7: SIMS profile comparing oxygen and carbon impurity concentrations for standard and windowed growths

There are three primary areas of interest in this plot. First, it is noted that there is a very high oxygen concentration in the stress-relief layers of the windowed growth wafers, with values exceeding $2 \times 10^{20} \mathrm{~atm} / \mathrm{cm}^{3}$, compared to $8 \times 10^{18} \mathrm{~atm} / \mathrm{cm}^{3}$ in the standard growth (region 3). During the early stages of growth, the side of the $\mathrm{SiO}_{2}$ sacrificial layer is exposed, resulting in $\mathrm{O}$ contamination. As the growth continues, the stress relief layer eventually covers the sacrificial layer, stopping the exposure of the oxygen to the HEMT layers.

Second, the buffer layer, which is the primary region of interest for oxygen incorporation, has very similar levels of carbon and oxygen compared to the standard growth. Elevated levels of carbon are found in regions 1 and 2, both close to the undoped GaN, as shown in Figure 4.7. These increased levels should not affect the 
performance of the devices, as the buffer layer is the region of more critical importance. The primary source of oxygen contamination is thought to be the methane source (99.99\% pure) used for carbon doping. This is shown in the SIMS profile as the carbon and oxygen concentrations mirror one another. Other sources of oxygen impurities include residual oxygen from the MBE chamber and the ammonia source.

\subsection{Silicon Surface Properties}

Auger spectroscopy is used at the silicon surface to verify that the sacrificial layer is fully lifted off, and that the growth process has not left surface contamination. Atomic force microscopy (AFM) results show an RMS roughness of $0.12 \mathrm{~nm}$ on a $5 \times 5 \mu \mathrm{m}^{2}$ area of the silicon surface, and $0.17 \mathrm{~nm}$ on a $25 \times 25 \mu \mathrm{m}^{2}$ region. This demonstrates the effectiveness of this technique in preserving a smooth silicon surface during the GaN epitaxy, as the roughness stays fairly consistent as the scan area is increased. The surface roughness as quoted by the manufacturer for new wafers is below $1 \mathrm{~nm}[67]$. Etching the GaN layers and then smoothing the silicon surface would not allow for this level of smoothness.

\subsection{Silicon $<110>$}

As discussed in Section 2.1.1, <110> oriented substrates are of increasing interest for state-of-the-art CMOS fabrication. Windowed growth on these substrates has also been achieved by making some slight alterations to the growth procedure described in 4.2 .1 .

The primary difference in growth technique is to initiate the growth of the first AlN buffer layer at a higher temperature $\left(820^{\circ} \mathrm{C}\right)$ and at a very low ammonia flow $(1 \mathrm{sccm})$. Once one monolayer is formed, the growth is briefly interrupted and the temperature 


\begin{tabular}{|c||c|c|c|c|c|c|c|}
\hline Property & $479 \mathrm{LA}$ & $479 \mathrm{LB}$ & $455 \mathrm{LA}$ & $455 \mathrm{LB}$ & $455 \mathrm{LC}$ & $455 \mathrm{LD}$ & $455 \mathrm{LE}$ \\
\hline Sheet Resistance $[\Omega / s q]$ & 538 & 516 & 693 & 556 & 657 & 718 & 1041 \\
Mobility $\left[\mathrm{cm}^{2} / \mathrm{Vs}\right]$ & 812 & 961 & 469 & 693 & 606 & 487 & 396 \\
$\begin{array}{c}\text { Sheet Carrier } \\
\text { Concentration }\left[\mathrm{cm}^{-2}\right]\end{array}$ & $1.30 \times 10^{13}$ & $1.27 \times 10^{13}$ & $1.93 \times 10^{13}$ & $1.62 \times 10^{13}$ & $1.57 \times 10^{13}$ & $1.78 \times 10^{13}$ & $1.51 \times 10^{13}$ \\
\hline
\end{tabular}

Table 4.2: Comparison of the properties of 110 windowed growths. 479L batch grown at NRC, 455L batch grown at CRHEA. Note that due to the windowed growth geometry, numbers are not exact, but can be used to compare between substrates

and ammonia flow are both ramped up to $930^{\circ} \mathrm{C}$ and $200 \mathrm{sccm}$ respectively. For the case of $<111>$ substrates, the initial temperature is much lower $\left(600^{\circ} \mathrm{C}\right)$ and both the flow and temperature are ramped up gradually. This difference in growth is needed, as the lattice mismatch is much larger with silicon $<110>$ substrates, so care must be taken to form a high-quality 2-D AlN monolayer before growth can proceed [68]. Table 4.2 summarizes the properties of the two samples that were grown using this technique. It should be noted that due to the geometry of the windowed growth the values cannot be accurately measured and hence, should only be used as an indication of the quality compared to the other samples.

\subsection{Conclusion}

A windowed growth technique which exposes a smooth silicon surface after GaN HEMT layer growth has been developed and characterized. A 2-DEG with comparable electrical properties to full-wafer growths has been achieved for both C-doped and non C-doped samples. Auger spectroscopy and atomic force microscopy analysis have shown that the exposed silicon windows are impurity-free and very smooth, which are essential requirements for MOS integration. By contrast, etching of GaN results in defects at the silicon surface. Growth on both $<111>$ and $<110>$ oriented 
substrates has been achieved as well. The $<110\rangle$ orientation is of particular interest as it may allow for future state-of-the art MOS devices to be integrated, making this technology more industrially relevant. 


\section{Chapter 5}

\section{Process Design}

This chapter presents the detailed design and fabrication of the monolithic GaN/CMOS chip. The general strategy used to integrate the required process steps has been presented in Chapter 3. The design of the combined process will be discussed, starting with the development of the MOS process and followed by a description of the GaN process. Next, the back-end processing and metallization will be summarized. This Chapter concludes with a discussion of improvements needed for a second iteration of the process design, resulting from the first set of fabricated devices.

The process used to generate the final result shown in Figure 3.3 can be divided into four major steps: silicon surface preparation, MOS processing, GaN processing, and metallization. The silicon surface preparation technique was already presented in Chapter 4. Two different processes were developed for the NMOS devices in this work. Originally, a higher temperature isolated design was used, but due to issues with thermal decomposition of GaN, a more conservative enclosed device geometry without full isolation was used in order to obtain preliminary results. Next, a second iteration of the process was performed in order to achieve proper device isolation without a high thermal budget, as well as mitigate performance issues that were seen

in the first successful run. The GaN process design is rather straightforward, as the device characteristics are primarily defined by the growth scheme described in the 
previous chapter. The primary alteration to the device formation scheme developed at NRC was the development of a gold-free ohmic metallization, which is presented here.

\subsection{NMOS Design}

Although it would have been beneficial to have both PMOS and NMOS devices integrated in this chip, using strictly NMOS for this project greatly reduced the complexity, cost, and fabrication time. Altering the process flow to include PMOS devices is fairly straightforward and could be performed in future runs. An NMOS process flow which accommodates the GaN structures needed to be designed. Due to the thermal expansion mismatch between the silicon substrate and the GaN layers, as well as the decomposition of the GaN material at elevated temperatures, an NMOS process which minimizes the thermal budget was desired.

The NMOS process architecture deviates slightly from standard techniques due to the thermal limitations of the GaN material. In order to reduce the stress on the wafers during the process, the high temperature steps were kept to a minimum; this involved using a retrograde p-well, using a thin field oxide to reduce long furnace cycles, and minimizing the rapid thermal anneals by using a joint metallization. A possible local oxidation of silicon (LOCOS) NMOS process was drawn up based on previous work performed in the Carleton Microfabrication facility, as well as Tsuprem4 [69] simulations to design the extra steps needed. Hand calculations to determine the proper threshold and doping levels were performed in the beginning, but quickly became inconsistent with TSuprem4 simulation results. Medici simulations were also performed to determine the transistor characteristics, and subsequently to develop a circuit model. An example Tsuprem4 input file for this process is presented in appendix A. 
The outline of the proposed NMOS process flow can be found in Figure 5.1. The MOS processing steps began with a wafer that had the GaN HEMT layers in place, as well as a smooth silicon surface. It should be noted that metallization took place at the end of the process, so the MOS circuitry is formed and then insulated with borophosphosilicate glass (BPSG) before the GaN devices are formed.
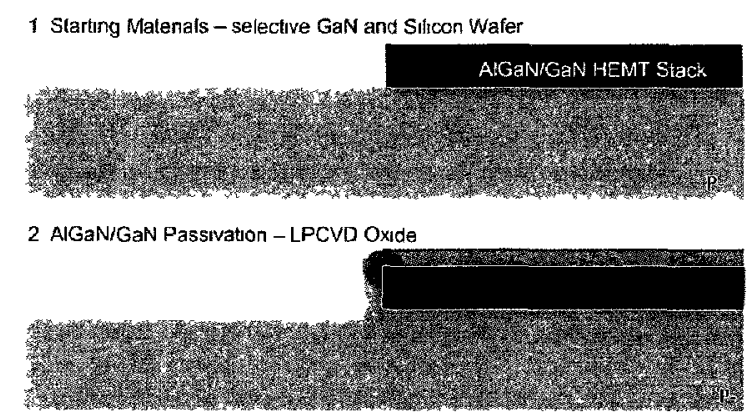

6 Gate Oxidation $-25 \mathrm{~nm}$ Dry 02
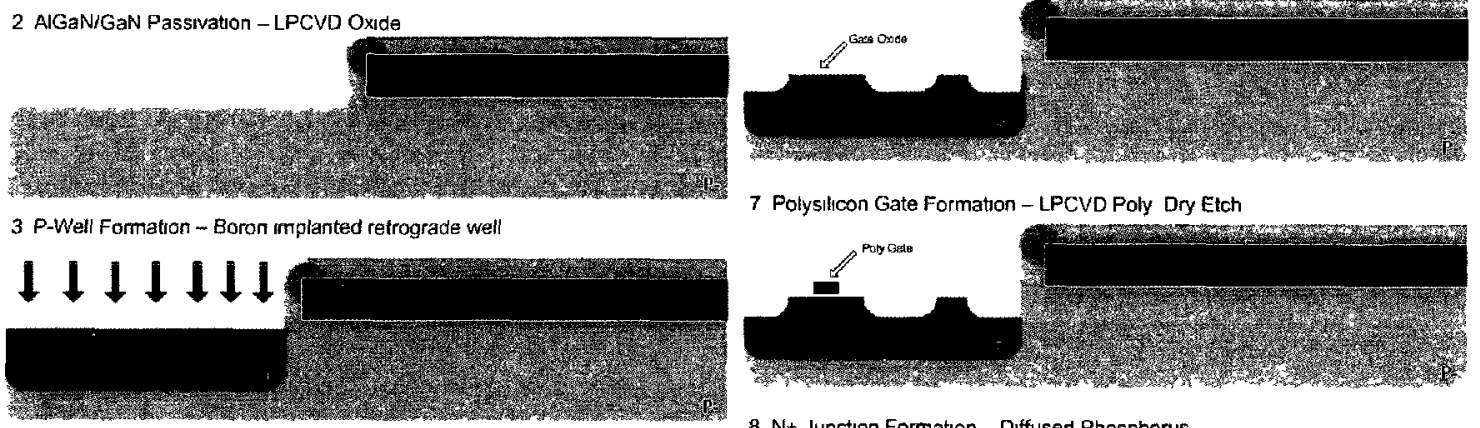

7 Folysilicon Gate Formation - LPCVD Poly Dry Etch

4 Active Region Formation - Pad Oxide and Nitnde
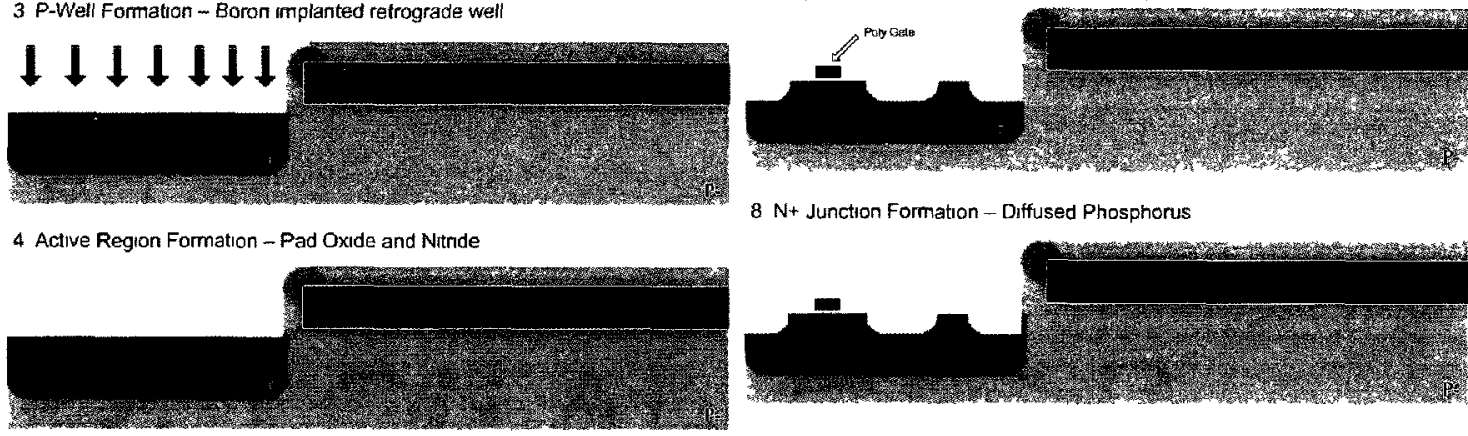

$8 \mathrm{~N}+$ Junction Formation - Diffused Phosphorus
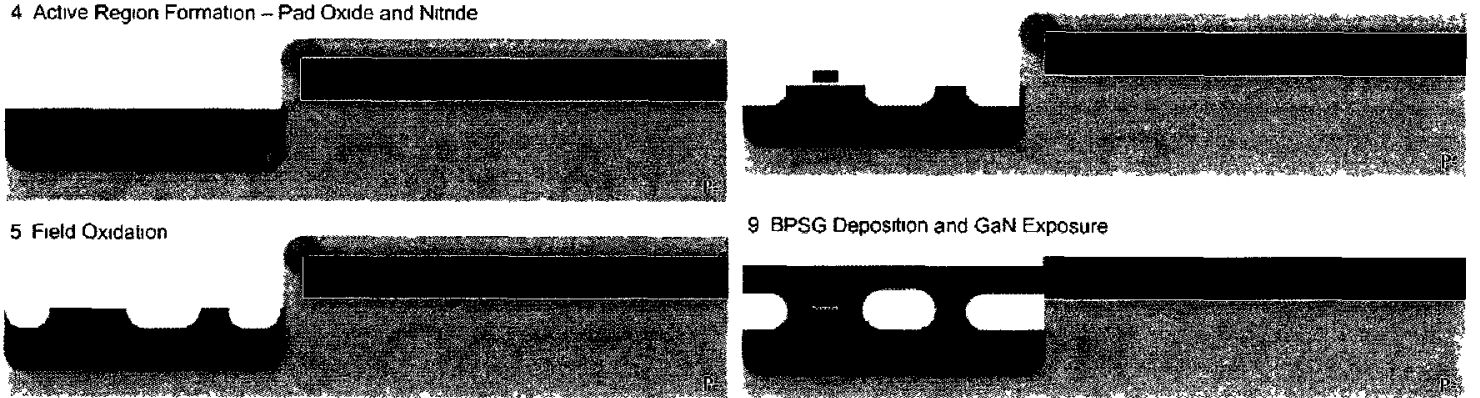

9 BPSG Deposition and GaN Exposure

Figure 5.1: MOS process steps

The designed NMOS process began with the formation of $\mathrm{p}$-wells, which were needed due to the high-resistivity substrates. In order to reduce thermal cycles, a 3 -implant retrograde well was formed. This allowed for a relatively uniform doping of $1 \times 10^{16} \mathrm{~cm}^{-3}$ and a depth of $1.3 \mu \mathrm{m}$ without the use of a drive-in diffusion. The subsequent oxidation steps were used to activate and drive in the dopant such that no 
additional thermal steps needed to be used. Figure 5.2a shows the simulated boron concentration profile for this implantation technique.

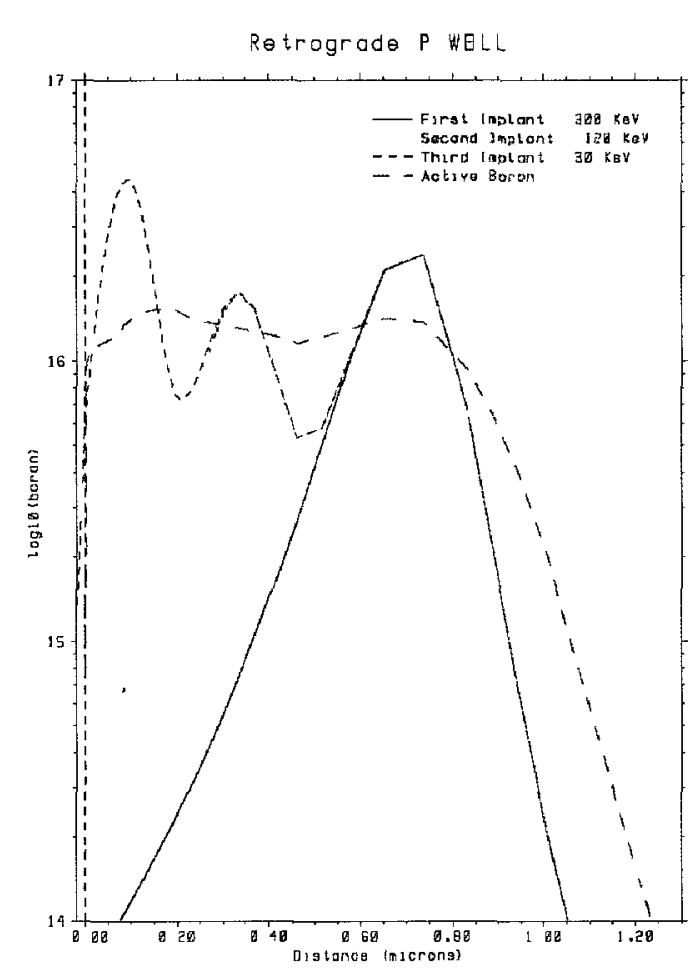

(a) Retrograde P-well Profile

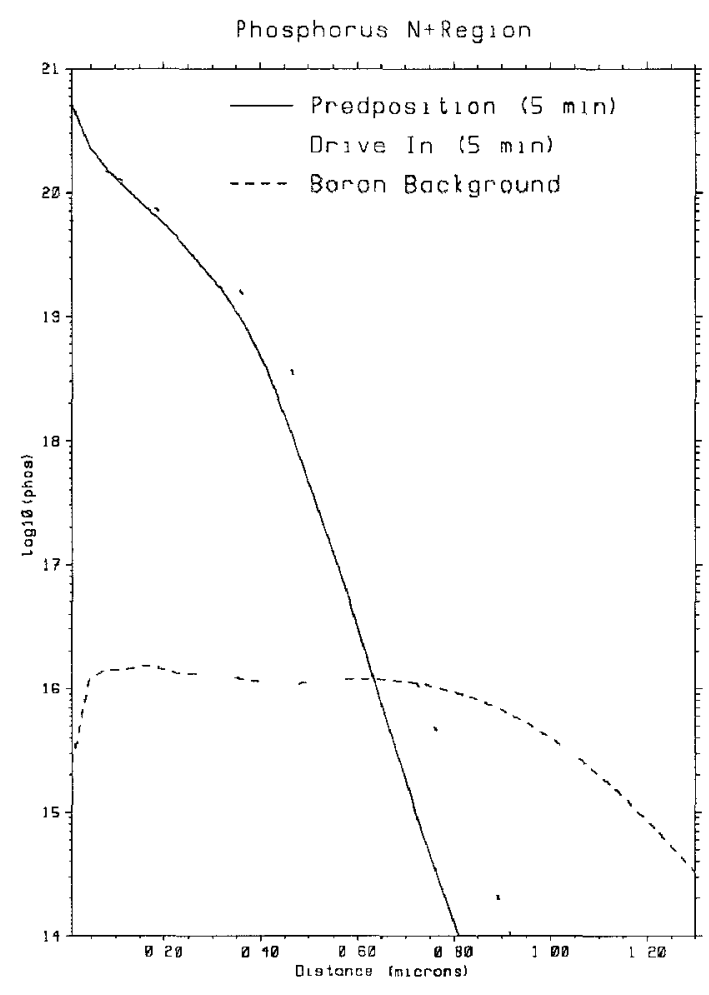

(b) N+ Junction Profile

Figure 5.2: Doping profiles from Tsuprem4 simulation

The thermal cycles from the field oxidation and gate oxidation were enough to activate the boron, eliminating the need for an implant anneal. A $0.5 \mu \mathrm{m}$ thick field oxide was chosen in order to keep the thermal budget as low as possible while still providing sufficient device isolation. An optional p-guard implant may be needed to raise the threshold voltage of the parasitic field devices in the NMOS process. A low energy boron implant of $4 \times 10^{13} \mathrm{~cm}^{-2}$ was demonstrated in simulation to raise the parasitic threshold voltage to approximately $17.5 \mathrm{~V}$ to ensure device isolation.

A gate oxide thickness of $25 \mathrm{~nm}$ was chosen in order to give good saturation 
drain current flow, set an appropriate threshold voltage $\left(V_{t}\right)$, as well as minimize thermal stress by minimizing the growth time. Simulations showed that a 13 minute oxidation using a dry $\mathrm{O}_{2}$ ambient at $1000^{\circ} \mathrm{C}$ would accomplish this. However, due to material decomposition, lower temperature oxidations of $950^{\circ} \mathrm{C}$ and $900^{\circ} \mathrm{C}$ were used. This will be discussed further in Section 6.3. According to Tsuprem4, the resulting threshold voltage would be $0.375 \mathrm{~V}$, which is appropriate for a supply of 3-5 V. If needed, a threshold adjust implant could be easily implemented in this process as part of the retrograde well formation.

$\mathrm{N}+$ junctions were formed with diffused phosphorus using a $\mathrm{POCl}_{3}$ process that has been developed at Carleton University. The diffusion is typically performed for 30 minutes at a temperature of $950^{\circ} \mathrm{C}$. According to simulation, this process results in a junction depth of $0.8 \mu \mathrm{m}$ and a sheet resistance of $32 \Omega / s q$. However, since the tube $\mathrm{POCl}_{3}$ concentration cannot be accurately specified, the Tsuprem4 simulation is, as a result, not completely accurate. The manner in which the pre-deposition was simulated was to assume a tube concentration such that the silicon is doped to solid solubility. Sheet resistances have typically been in the $10 \Omega / s q$ range in past runs performed at slightly higher temperatures. The plot in Figure 5.2b shows the 1-D junction profile as predicted by Tsuprem4 simulation.

\subsubsection{Enclosed MOS Devices}

Because initial trial runs of the LOCOS process caused GaN decomposition, a simpler process was developed to yield NMOS and GaN devices on the same chip without the added thermal budget needed to isolate MOS devices.

MOS devices were fabricated and tested by completely enclosing a drain region with a gate, which is entirely enclosed by a source region. This is shown in crosssection in Figure 5.3 and in plan view in Figure 5.4. Note that the current flow goes from the source to the enclosed drain such that it does not interfere with other 


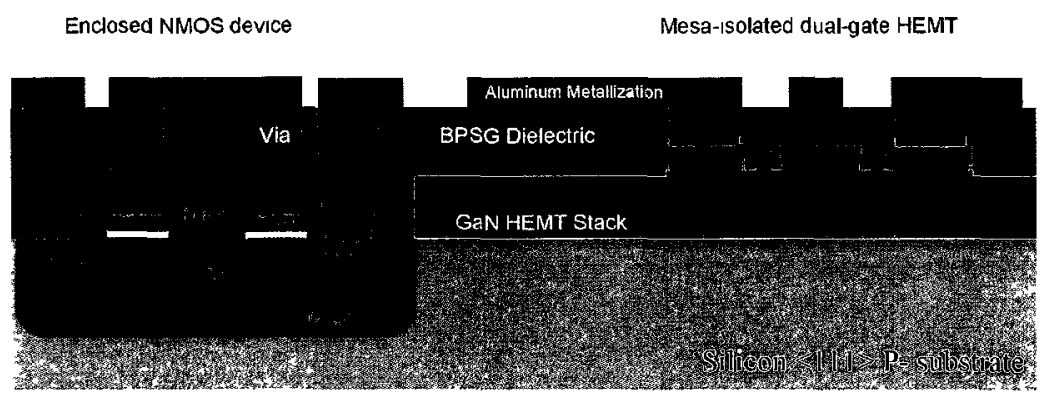

Figure 5.3: Cross-Section of enclosed nMOS device

devices.

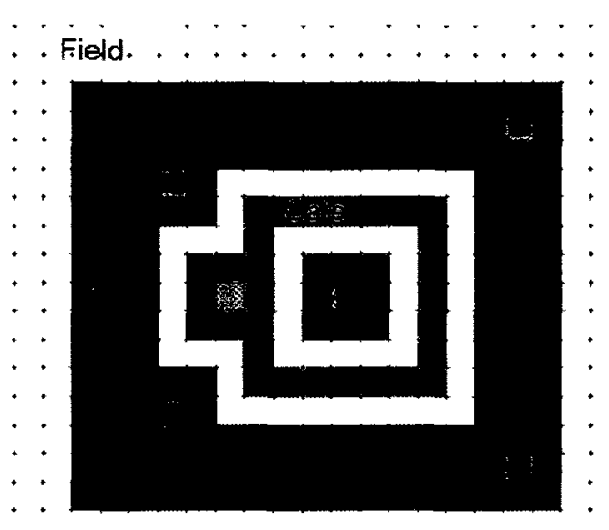

Figure 5.4: Layout of an enclosed NMOS device

Only relatively wide MOSFETs can be fabricated with this approach. This precludes the fabrication of complex circuits, but does suffice to demonstrate the cointegration concept without the added complexity and thermal stress required for the LOCOS process. The process shown in Figure 5.1 was altered such that gate oxidation simply followed the well implant. The pad oxidation, p-guard (if needed), and field oxidation steps were all eliminated. The device results are presented in Section 6.1 . 


\subsubsection{Electrical Characteristics}

2-D Medici simulations were used to predict the NMOS characteristics. The input code for the Medici simulations can be found in Appendix B. Profiles for each portion of the device were imported into Medici from Tsuprem4 in order to give an exact representation of the device structure. The IV curves for a $1 \mu \mathrm{m}$ wide and $2.5 \mu \mathrm{m}$ long device are shown in Figure 5.5. It should be noted that the $2.5 \mu \mathrm{m}$ gate length is the lithographic limitation for this process. To scale down the process further, implanted source/drain regions will be needed as short-channel effects caused by the relatively deep diffusions will interfere with device performance. Device dimensions on the order of $1 \mu \mathrm{m}$ should be achievable with a shallow implanted arsenic junction.

Parameter extraction revealed the device had a $0.375 \mathrm{~V}$ threshold voltage and a sub-threshold swing of $79.3 \mathrm{mV} /$ decade. This value of sub-threshold swing is quite reasonable, and suggests that channel leakage at $\mathrm{V}_{G}=0 \mathrm{~V}$ will not be a problem. Since the sub-threshold curves for different $\mathrm{V}_{D S}$ almost super-imposed (Figure 5.5b), it can be concluded that drain-induced barrier lowering is not an issue. The good sub-threshold characteristics can be attributed to the long channel and thin gate oxide.

Punch-through is a short-channel effect which occurs when the gate is unable to shut off the drain current. A criterion for punch-through of $10 \mathrm{nA} / \mu \mathrm{m}$ drain current at $\mathrm{V}_{G S}=0$ was chosen here. A simulation which varies $\mathrm{V}_{D S}$ as the gate voltage is kept at zero suggested that the punch-through voltage for this design is in excess of $40 \mathrm{~V}$. Figure 5.5c, which shows a plot of off-state current vs. drain voltage, illustrates this result.

Hot carrier effects were examined by comparing the drain and substrate current, and the maximum drain voltage at the gate voltage which gives the highest avalanche generation. With the drain voltage set, the largest avalanche generation occurs at 


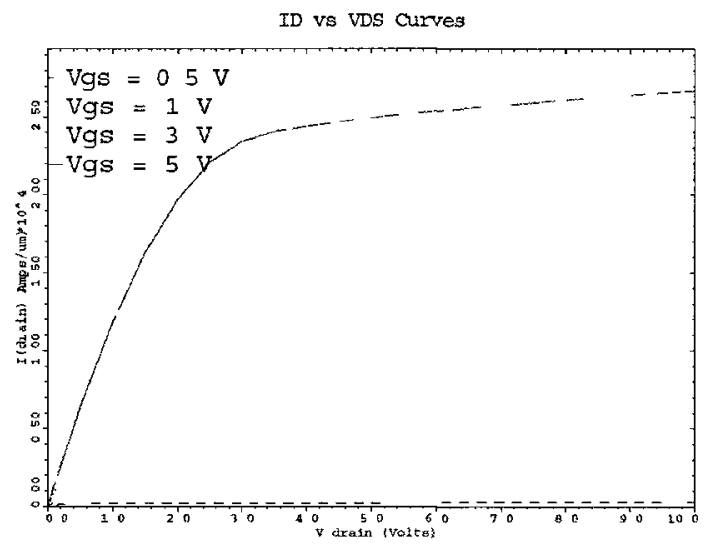

(a) Forward Drain Current

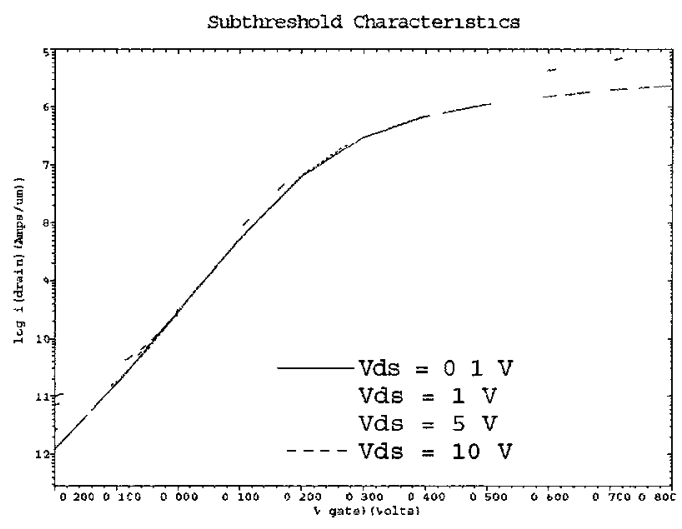

(b) Subthreshold Characterıstıc

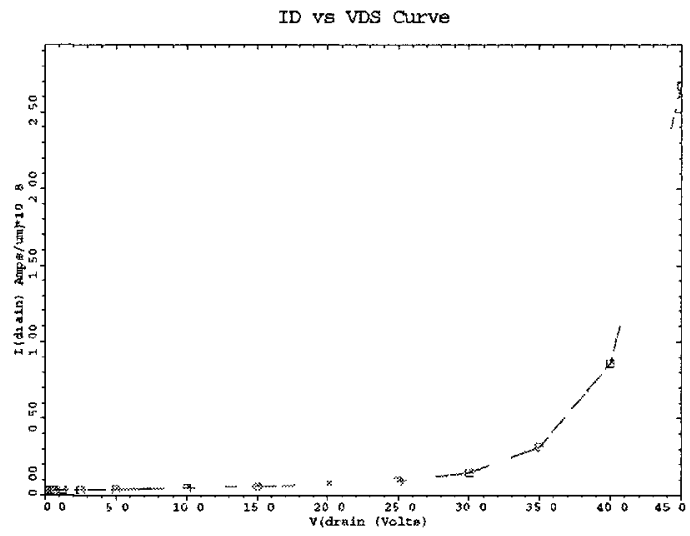

(c) Offstate Leakage $\left(\mathrm{V}_{G S}=0\right)$

Figure 5.5: Medici simulations of MOS electrical characteristics for a $2.5 \mu \mathrm{m}$ long device

a gate voltage of approximately $3 \mathrm{~V}$. Simulations show that the substrate current accounts for less than $1 \%$ of the drain current, suggesting that hot carrier effects should not have been seen in these devices.

\subsection{GaN Devices}

The GaN HEMT process used was based on one already developed at NRC. The properties of the device are primarily dictated by the quality of the epitaxial growth 
and hence, the primary engineering contribution with respect to the GaN devices was the development of the windowed growth technique presented in Chapter 4 . Some alterations to the existing process were required in order to make it compatible with MOS fabrication. The primary change that was made is the metallization scheme. Gold is traditionally used for GaN device fabrication, but it can interfere with MOS circuitry due to the introduction of a mid-level trap, which can cause leaky junctions [70]. An alternative ohmic contact scheme based on aluminum was developed. Figure 5.6 outlines the GaN processing steps.



Figure 5.6: GaN processing steps

Starting with the passivated MOS circuitry shown in Figure 5.1, the first step was to etch the mesas for device isolation. A chemically-assisted ion beam etch (CAIBE) with a $\mathrm{Cl}_{2} / \mathrm{O}_{2} / \mathrm{Ar}$ chemistry is used to form a slanted MESA edge, and to etch down to below the 2-DEG. Next, ohmic contacts were formed with an evaporated $\mathrm{Ti} / \mathrm{Al} / \mathrm{Ti} / \mathrm{Al}$ stack rather than a $\mathrm{Ti} / \mathrm{Al} / \mathrm{Ti} / \mathrm{Au}$ stack, which is traditionally used. Following the ohmic metal, a Pt-based gate metallization step takes place. The device was then 
passivated with a thin PECVD nitride layer in order to mitigate surface traps which cause current collapse [9]. At this point, the wafer was ready for back-end processing and metallization.

\subsection{Metallization and Passives}

\subsubsection{Metallization}

In order to save on steps and mask costs, the metallization for both MOS and GaN devices was done concurrently. A lift-off technique based on the GaN process was used for this step as it provides a superior edge profile and eliminated the need for a metal etch step.

Figure 5.7 shows the metallization strategy used to connect the chip. By using a combined metal for both types of devices, not only is the mask cost lowered, the thermal budget is also reduced by eliminating extra RTA steps.

Again, aluminum was used in place of gold in order to make the process compatible with MOS technology. As long-term operation was not a primary objective at this point, a simple metallization scheme was desired. The metal1 and metal2 layers were simply a thin titanium layer followed by thick aluminum. The role of the titanium layer was to aid adhesion to the silicon.

\subsubsection{Passive Components}

Although the main goal of this work was to integrate active devices of both types, some passive components were also included for testing as they would be needed for future circuit applications. Integrated polysilicon test resistors were included for simplicity. There is also a possibility to include NiCr resistors as these were used for the GaN process. Spiral inductors were also included and designed using 


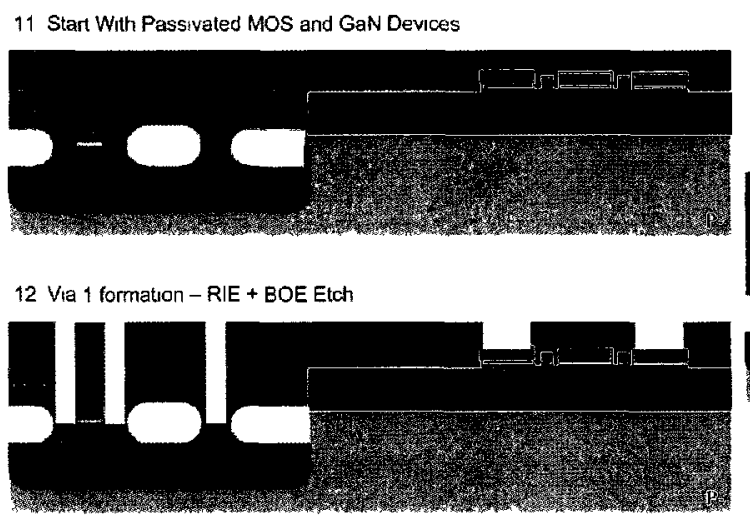

14 Metal 2 Passivation - Floweo BPSG
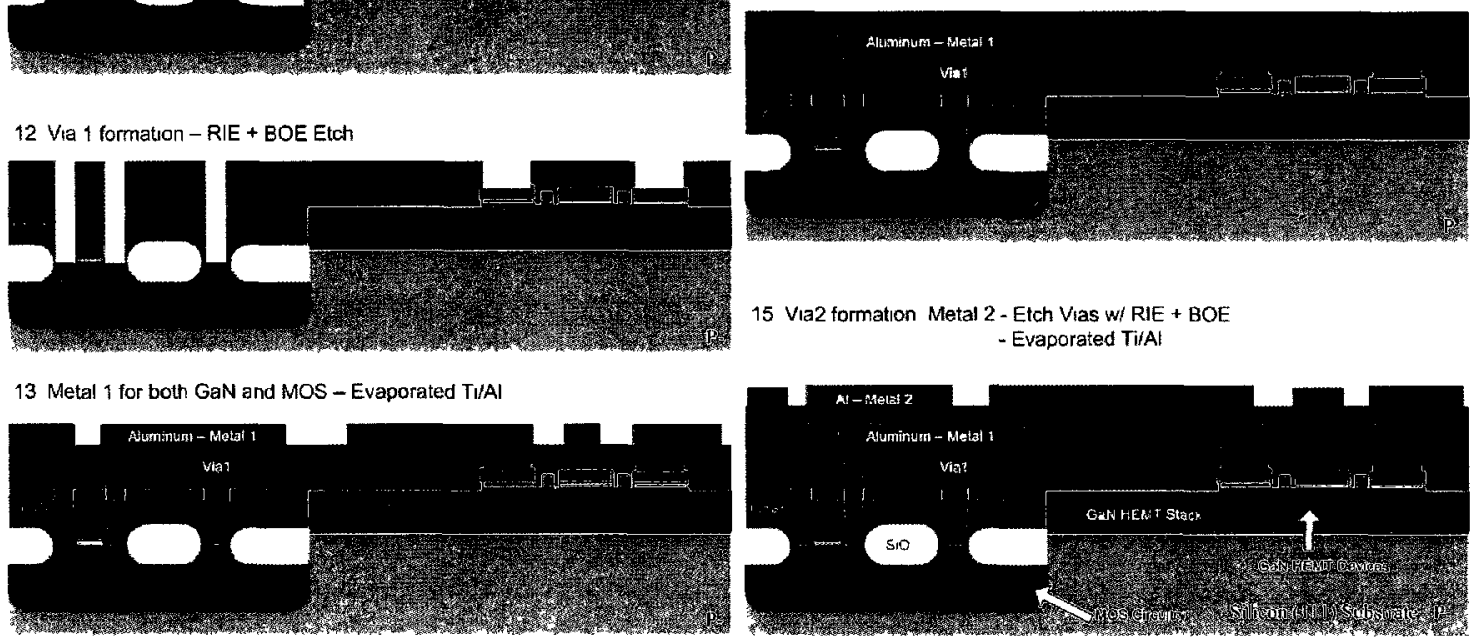

Figure 5.7: Metalization process

Sonnet [71] for operation in the $\mathrm{GHz}$ range, but were not tested as the samples never reached this step in the process. These were simply formed using the top metal layer of the process. Test MOScaps were also included.

\subsection{Development of a Gold-Free Ohmic Contact}

The formation of ohmic contacts to $\mathrm{AlGaN} / \mathrm{GaN}$ heterostructures has always been a challenge due to the large band-gap of this material system. Traditional strategies to overcome the resulting large Schottky barrier height have been to use multi-layer metal stacks annealed at high temperatures, based on a low work-function metal such as titanium [72]. This is because the inter-metallic alloys formed during hightemperature anneals lower the work function of the metal, and hence reduce the potential barrier. Tunnelling through this lowered potential barrier is the primary mechanism through which current passes through the ohmic contact. It is generally 
believed that nitrogen vacancies are created through the interaction of GaN with the contact metal, resulting in n-type doping. This is the physical mechanism by which low resistance ohmic contacts are formed in GaN.

Early work in ohmic contacts revolved around the use of $\mathrm{Ti}$, as it has a low work function. However, the unreliability of $\mathrm{Ti}$ or $\mathrm{Ti} / \mathrm{Al}$ bilayers due to oxidation and the low melting point of $\mathrm{Al}$ have necessitated more complicated multilayer metal systems, most commonly $\mathrm{Ti} / \mathrm{Al} / \mathrm{Ti} / \mathrm{Au}$ and $\mathrm{Ti} / \mathrm{Al} / \mathrm{Ni} / \mathrm{Au}$ [73]. The $\mathrm{Au}$ is employed as the top layer metal due to its low electrical resistance, its resistance to electromigration, and to improve the oxidation resistance of the $\mathrm{Ti} / \mathrm{Al}$ bilayer. The barrier metal ( $\mathrm{Ti}$ or $\mathrm{Ni}$ ) was proposed to block diffusion of the $\mathrm{Au}$ into the $\mathrm{Ti} / \mathrm{Al}$ layers, as well as to provide additional $\mathrm{Ti}$ for the formation of intermetallics such as $\mathrm{Ti}_{3} \mathrm{Al}$ and $\mathrm{Al}_{3} \mathrm{Ti}[73]$. Nevertheless, Auger profiling of alloyed contacts shows that these layers are often not effective as diffusion barriers.

In order to mitigate the effects of Au contamination in MOS devices, an Aufree ohmic contact was developed for this work. Two alternatives to the schemes described above are $\mathrm{Ti} / \mathrm{Al} / \mathrm{Ti} / \mathrm{Al}$ and $\mathrm{Ti} / \mathrm{Al} / \mathrm{Ti} / \mathrm{Ag}$. The use of an aluminum top layer posed concerns over oxidation, but was a good short-term solution while more reliable contacts were being developed. Silver is currently of interest for use in ohmic contacts as it has a lower work function than gold, as will silver-based inter-metallic phases, facilitating a low-resistance contact [74]. Additionally, silver has the lowest resistivity of all metals $\left(1.6 \times 10^{-8} \Omega \cdot \mathrm{m}\right.$ vs $2.2 \times 10^{-8} \Omega \cdot \mathrm{m}$ for gold $)$, making it a material of interest for improved interconnects and metallization.

Based on the work of Miller et al. [74], a $\mathrm{Ti} / \mathrm{Al} / \mathrm{Ti} / \mathrm{Ag}$ ohmic contact was attempted on windowed growth material, resulting in comparable characteristics to $\mathrm{Ti} / \mathrm{Al} / \mathrm{Ti} / \mathrm{Au}$ contacts and $\mathrm{Ti} / \mathrm{Al} / \mathrm{Ti} / \mathrm{Al}$ contacts. These contact schemes were tested on samples of the first windowed growth wafer. Unfortunately, the results in this looked promising, but were limited in quantity due to two primary factors. Firstly, 
the amount of material was limited as only one wafer was available for characterization of windowed growth material, as others were left for full processing. Secondly, cracking during the ohmic contact formation resulted in the loss of some samples, most likely due to stress. This is discussed in 5.4.2. Thus, Ti/Al/Ti/Al contacts were used in subsequent runs as not enough material was available to optimize the ohmic contact.

\subsubsection{Fabrication and Characterization of Ohmic Contacts}

Ohmic metal is formed using e-beam evaporation and a lift-off process developed at NRC. This is followed by a rapid thermal anneal, typically between $700^{\circ} \mathrm{C}$ and $950^{\circ} \mathrm{C}$. These high temperatures are needed to overcome the wide bandgap, and hence large work function of the $\mathrm{AlGaN} / \mathrm{GaN}$ material. The $\mathrm{Ti} / \mathrm{Al} / \mathrm{Ti} / \mathrm{Al}$ ohmic contacts in this work were annealed between $845^{\circ} \mathrm{C}$ and $875^{\circ} \mathrm{C}$.

Characterization of the ohmic contacts was performed using circular Corbino patterns during processing, as well as Transmission Line Method (TLM) patterns, which were measured after the first metallization step. Table 5.1 summarizes the ohmic contact properties for all samples fabricated and tested.

\begin{tabular}{|c||c|c|}
\hline Sample & Contact Resistance & Sheet Resistance \\
\hline 464LA & $2.03 \Omega-\mathrm{mm}$ & $399 \Omega / s q$ \\
$484 \mathrm{LC}$ & $8.28 \Omega-\mathrm{mm}$ & $415 \Omega / s q$ \\
$487 \mathrm{LA}$ & $4.18 \Omega-\mathrm{mm}$ & $393 \Omega / s q$ \\
$487 \mathrm{LB}$ & $6.53 \Omega-\mathrm{mm}$ & $1836 \Omega / s q$ \\
$488 \mathrm{LA}$ & $3.59 \Omega-\mathrm{mm}$ & $425 \Omega / s q$ \\
$488 \mathrm{LB}$ & $5.35 \Omega-\mathrm{mm}$ & $348 \Omega / s q$ \\
\hline
\end{tabular}

Table 5.1: Ohmic contact results as measured by TLM patterns following fabrication 
It can be seen from this that the first run of the Al-based ohmic in sample 464LA gave the best results. The second iteration of processing devices resulted in inferior contacts, most likely due to three factors: the Al layer was found to be more oxidized (as observed through microscope inspection) in this case compared to the 464L batch;

a thin oxide layer was left on the wafers before metal deposition on some samples; and the material quality of some of the later samples was not as good, resulting in a much worse contact as well as sheet resistance. Because of the limited availability of starting material, a full optimization of this ohmic contact scheme could not be performed.

\subsubsection{Stress in Ohmic Contacts}

The high temperature anneal as well as thick metal stack required to form high quality ohmic contacts to $\mathrm{AlGaN} / \mathrm{GaN}$ are a major source of stress on the heterostructure during fabrication. Cracking of the GaN layers during this anneal step during ohmic contact formation is quite common, especially in growths on silicon substrates, as overcoming the thermal expansion mismatch remains a challenge. It is difficult to predict whether or not a wafer would crack as it is largely related to the quality of the growth and stress relief layers.

Figure 5.8 shows a crack in the gate periphery of a device due to the stress caused by the ohmic contact anneal.

\subsection{Mask Layout}

The integration strategy used in this work for topographical layout was to make use of an existing GaN mask set in conjunction with new masks that include MOS circuitry. As the NRC mask set consisted of an array of test structures, it was simple to block out one of every four units and replace it with an area for MOS fabrication. 


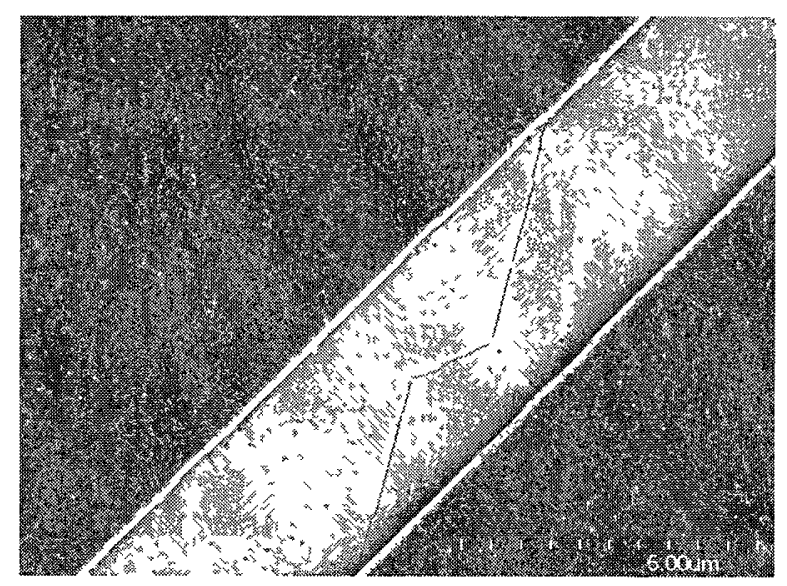

Figure 5.8: SEM micrograph of crack formation in the gate periphery of a device due to ohmic contact anneal (sample 456LB)

Two sets of NMOS masks were used for this work. The first set was based on a more ambitious MOS technology, and included test devices, several different circuits as well as passives such as poly resistors, spiral inductors, and MOScaps. The minimum geometry of the MOS devices in this case was $2.5 \mu \mathrm{m}$. When diffuculties were encountered in GaN HEMTs surviving the thermal budget required for full MOS processing, an enclosed MOS mask set was also produced, which only included enclosed MOS transistors and had a minimum geometry of $12.5 \mu \mathrm{m}$.

The advanced design had 13 mask levels, which are listed below.

\section{GaN Isolation Covers GaN regions}

Silicon Isolation Covers silicon regions

Device Well Device Well for MOS devices

Poly Polysilicon for MOS devices

Mesa (GaN) GaN Mesa

Ohmic (GaN) GaN Ohmics 
Gate (GaN) GaN Gates

Contact MOS contacts to poly and device well

Via (GaN) GaN Vias to ohmic and gate metal

Metal 1 (GaN) First metal pattern for GaN

Metal 1 First metal for MOS

Via 2 Via between Metal 1 and Metal 2 for MOS and GaN

Metal 2 (GaN) Second metal for GaN and MOS, used to connect devices together

Note that the Via 1 and Metal 1 layers were all dual dark field exposures, and hence performed in the same lithography step. The Via 2 and Metal 2 masks were made to cover the entire chip and were used to connect MOS and GaN devices for circuits. Because of process issues, the project was never taken to this level. One of the mask blocks showing the MOS area and GaN devices is shown in Figure 5.9.

On the left side, the MOS devices are connected to the GaN devices using Metal 2 to form circuits. The MOS devices, circuits, test structures, and passives are in the bottom left block.

The enclosed process was used to simplify fabrication, focussing only on obtaining working devices of both types on the same chip. The mask number was reduced to 10 , as listed below.

GaN Isolation Covers GaN regions

Silicon Isolation Covers Si regions

Poly Polysilicon for MOS devices

Mesa (GaN) GaN Mesa 


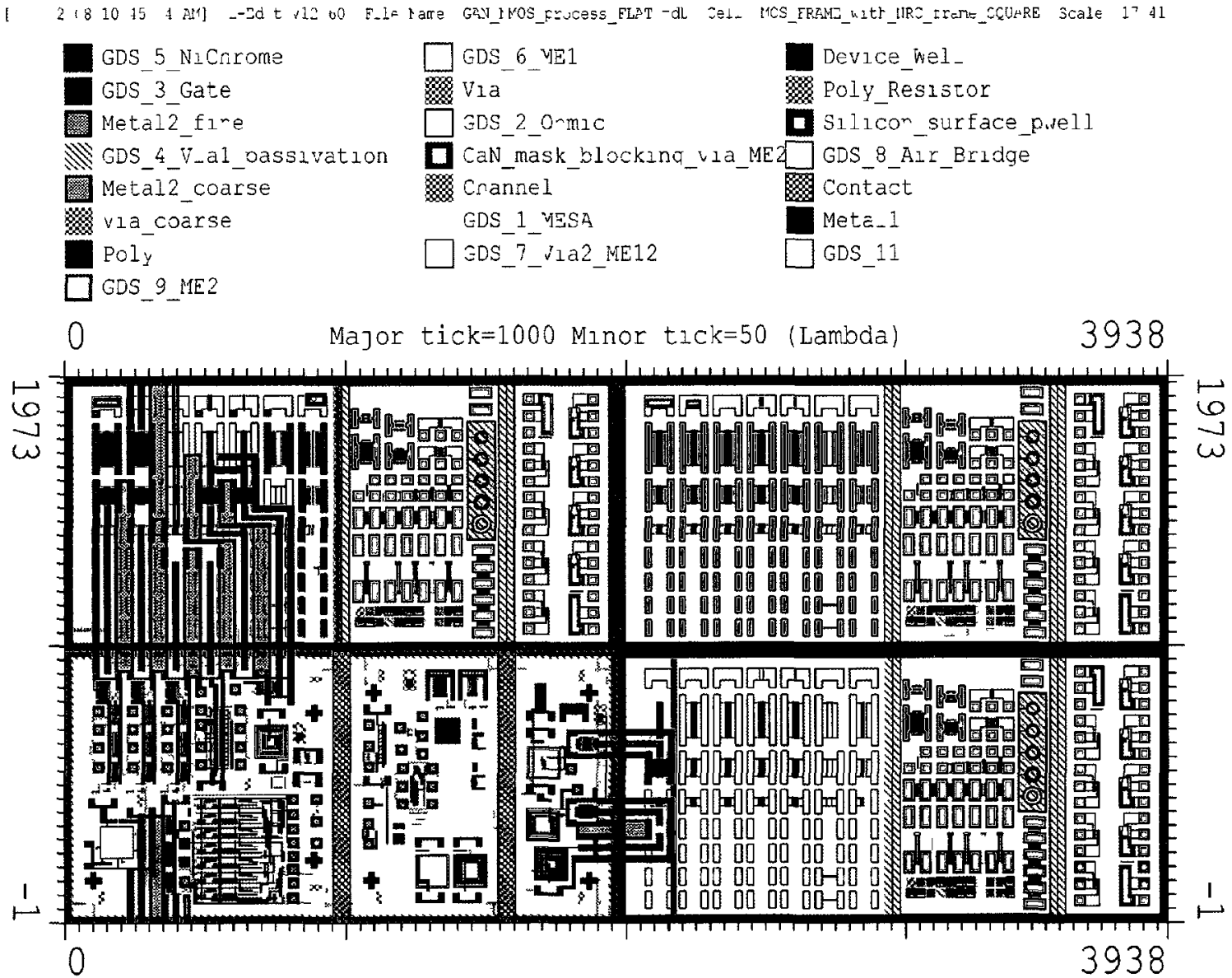

Figure 5.9: First mask layout showing MOS region (bottom left) as well as other GaN patterns 


\section{Ohmic (GaN) GaN Ohmics}

\section{Gate (GaN) GaN Gates}

Contact MOS contacts to poly and device well

Via (GaN) GaN Vias to ohmic and gate metal

Metal $1(\mathrm{GaN})$ First metal pattern for $\mathrm{GaN}$

Metal 1 First metal for MOS

The layout of the enclosed MOS device frame is shown in Figure 5.10. In this case, there is no connective metal between the GaN and MOS devices. The larger scale of the devices is also shown here as the masks for the enclosed devices were run directly off of the pattern generator and not stepped down to smaller dimensions in order to reduce complexity.

The GaN mask included arrays of dual-gate test transistors with a fixed gate length of $0.8 \mu \mathrm{m}$. These ranged in width from 10 to $320 \mu \mathrm{m}$. Additionally, there were Corbino and TLM patterns on the mask for measuring sheet resistance and ohmic contact resistance. The mask also included sets of nichrome resistors, and air-bridged devices. These, however, were not used for this project.

By using a printed mylar transparency sandwiched with the glass mask, in addition to patterned passivating oxide layers, selective areas of the wafers could be processed while other areas were left protected. The BPSG layer deposited after the MOS device well and polysilicon deposition was also used as a passivation layer during MOS fabrication. Conversely, a thick $1 \mu \mathrm{m}$ PECVD $\mathrm{SiO}_{2}$ layer is used to cover the GaN regions during MOS fabrication. This layer is removed before GaN fabrication using partial dry etching followed by wet etching to minimize damage to the GaN surface. After MOS and GaN device fabrication, RIE is used to etch through the 


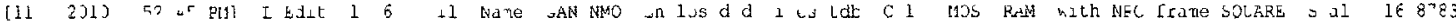

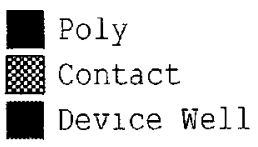

$\begin{array}{ll}\square \text { GDS_2_OhmıC } & \text { Metall } \\ \text { GDS_1_MESA } & \text { GDS_3_Gate } \\ \square \text { Silicon_surface pwell } \square \text { GDS_6 ME1 }\end{array}$

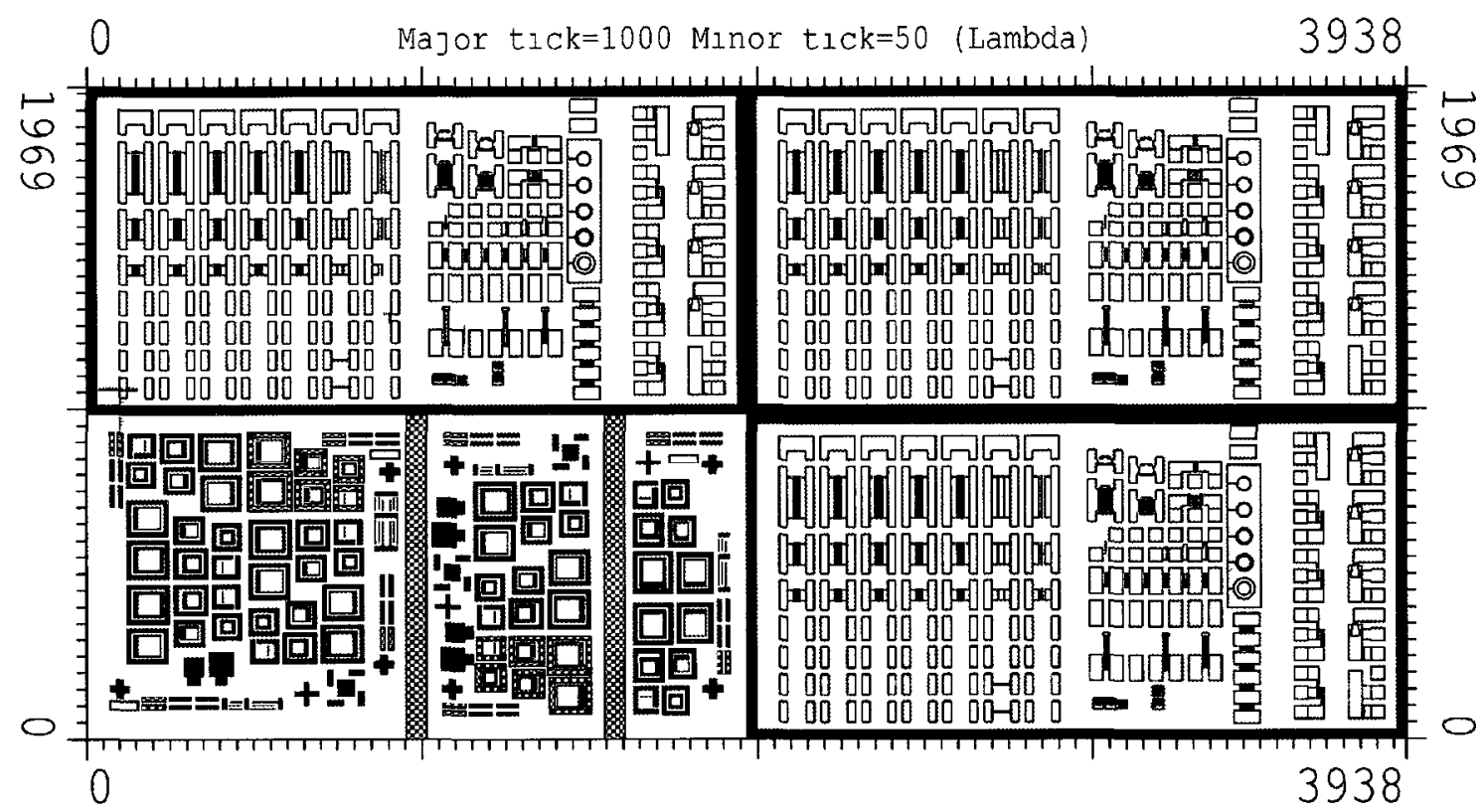

Figure 5.10: Enclosed mask layout showing MOS region (bottom left) as well as other GaN patterns 
GaN passivation as well as the BPSG layer to reach either poly, silicon, or GaN metal to make contact to the metal 1 layer.

\subsection{Second Iteration of MOS Design}

A second iteration of the process was designed and implemented in order to mitigate some of the MOS performance issues resulting from the initial run. Although the MOS devices were functional, they had two primary issues that hampered performance: gate leakage and drain leakage. Additionally, the enclosed geometry process did not allow for isolated devices. A new isolation scheme based on a thick deposited oxide layer was proposed to resolve these problems.

Drain leakage was caused by the deeper than expected source and drain junctions punching through the implanted P-well; this is shown in Section 6.1. The source and drain junctions were diffused longer than originally simulated in order to reduce sheet resistance; however, this also resulted in a deeper junction. A TSuprem4 simulation which matches the experimental result of $17.9 \Omega / s q$ is shown in Figure 5.11b. In this case, the depth of the $\mathrm{n}+$ junction is $1.2 \mu \mathrm{m}$, which is close to the depth of the P-well $(1.3 \mu \mathrm{m})$ shown in Section 5.1. The implant dose and energy of the P-well was

subsequently increased in order to form a deeper well as shown in Figure 5.11a. The new well should be approximately $2 \mu \mathrm{m}$ deep.

Table 5.2 summarizes the original and modified P-well implant scheme, which resulted in the same surface concentration as the original scheme in order to maintain the correct threshold voltage.

In order to reduce the thermal budget of the process flow, a new isolation technique based on a thick PECVD oxide was attempted, rather than using LOCOS. This scheme involved depositing a thick oxide layer, which is then subsequently wet etched to form the device well region. This isolation scheme was used in very early CMOS 


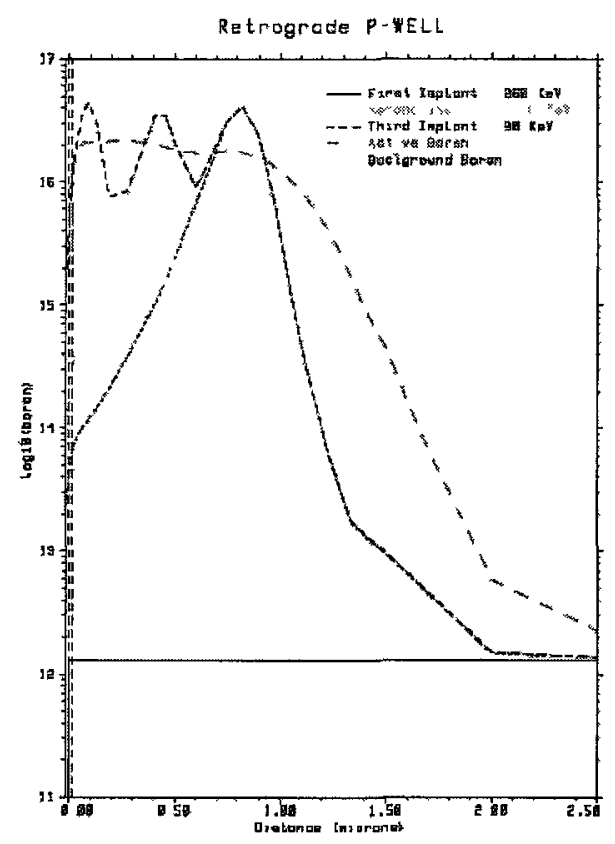

(a) Deeper Retrograde P-well Profile

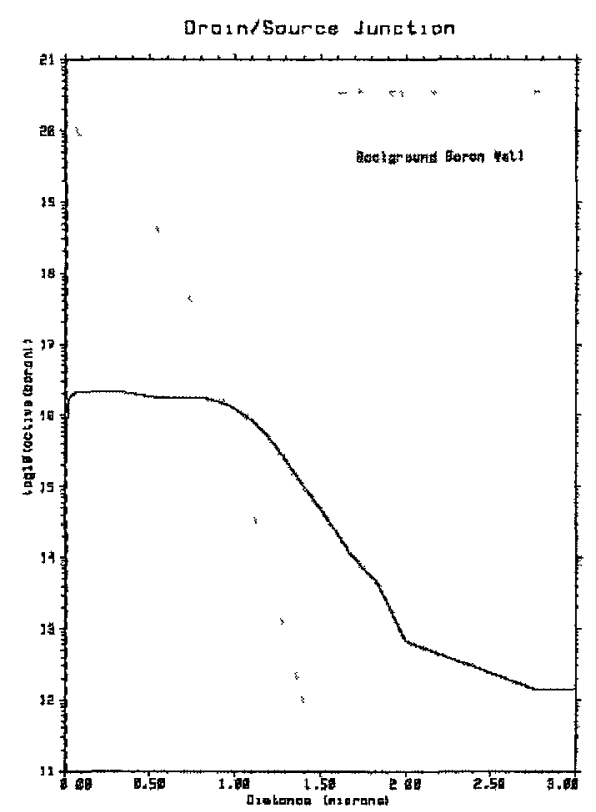

(b) Deeper N+ Junction Profile

Figure 5.11: Revised doping profiles from Tsuprem4 simulation

technologies. GaN decomposition was not a threat in this case as the PECVD layer was deposited at $500^{\circ} \mathrm{C}$. This process alteration allowed the $900^{\circ} \mathrm{C}$ based process to include isolated devices, and hence circuits, as opposed to the enclosed process. The first iteration of the MOS mask was used in this case. Figure 5.12 shows the crosssections of both the parasitic and normal MOS devices using both the LOCOS and thick oxide isolation schemes.

Attempts to grow the thermal oxide at $950^{\circ} \mathrm{C}$ were also made in the new iteration in order to eliminate the gate leakage. The enclosed mask was used in this case to eliminate the thermal budget needed for LOCOS isolation. Eventually, these techniques can be combined in order to fabricate a chip with isolated devices that also have a good quality gate oxide. An in-depth discussion of the oxidations can be found in Section 6.3. 


\begin{tabular}{|c||c|c|}
\hline Implant Property & Original Implant & Revised Implant \\
\hline Implant 1 & $6 \times 10^{11} 1 / \mathrm{cm}^{2} 300 \mathrm{kEv}$ & $1 \times 10^{12} 1 / \mathrm{cm}^{2} 360 \mathrm{kEv}$ \\
Implant 2 & $3 \times 10^{11} 1 / \mathrm{cm}^{2} 120 \mathrm{kEv}$ & $7 \times 10^{11} 1 / \mathrm{cm}^{2} 160 \mathrm{kEv}$ \\
Implant 3 & $5 \times 10^{11} 1 / \mathrm{cm}^{2} 30 \mathrm{kEv}$ & $5 \times 10^{11} 1 / \mathrm{cm}^{2} 30 \mathrm{kEv}$ \\
Surface Concentration & $1 \times 10^{16} 1 / \mathrm{cm}^{-3}$ & $1 \times 10^{16} 1 / \mathrm{cm}^{-3}$ \\
Depth & $1.3 \mu \mathrm{m}$ & $2 \mu \mathrm{m}$ \\
\hline
\end{tabular}

Table 5.2: Summary of P-well retrograde well implant parameters

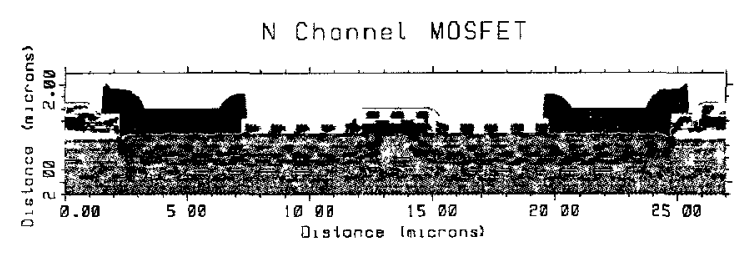

(a) NMOS LOCOS Isolation

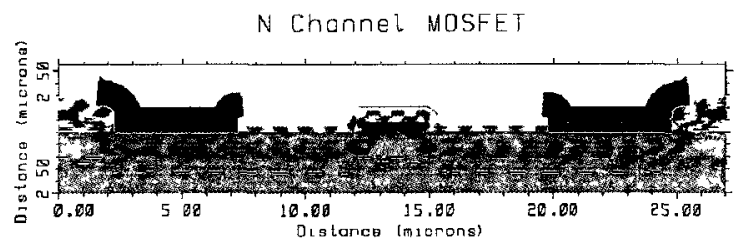

(c) NMOS Oxide isolated

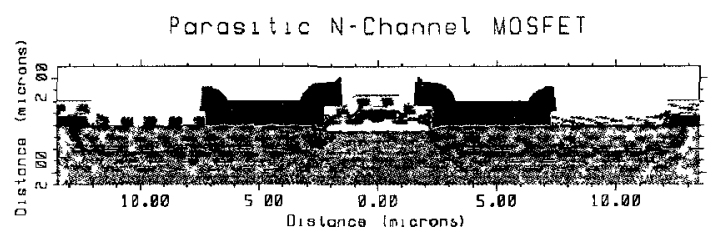

(b) NMOS Parasitic LOCOS isolation

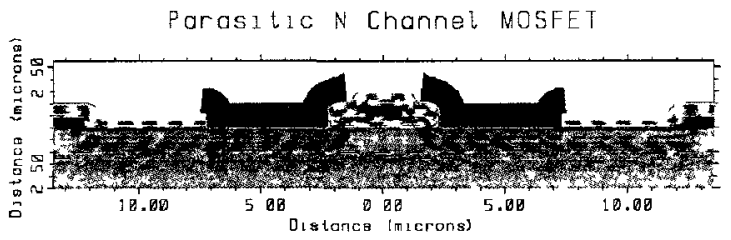

(d) NMOS Parasitic Oxide Isolation

Figure 5.12: MOS cross Sections showing both isolation techniques

Appendix $\mathrm{C}$ shows a full process specification for the integration process using the enclosed mask. This includes the minor changes made during the second iteration of processing. Figure 5.13 shows a SEM micrograph of the GaN devices integrated with MOS structures prior to final metallization for the enclosed mask and after completion of device isolation. 


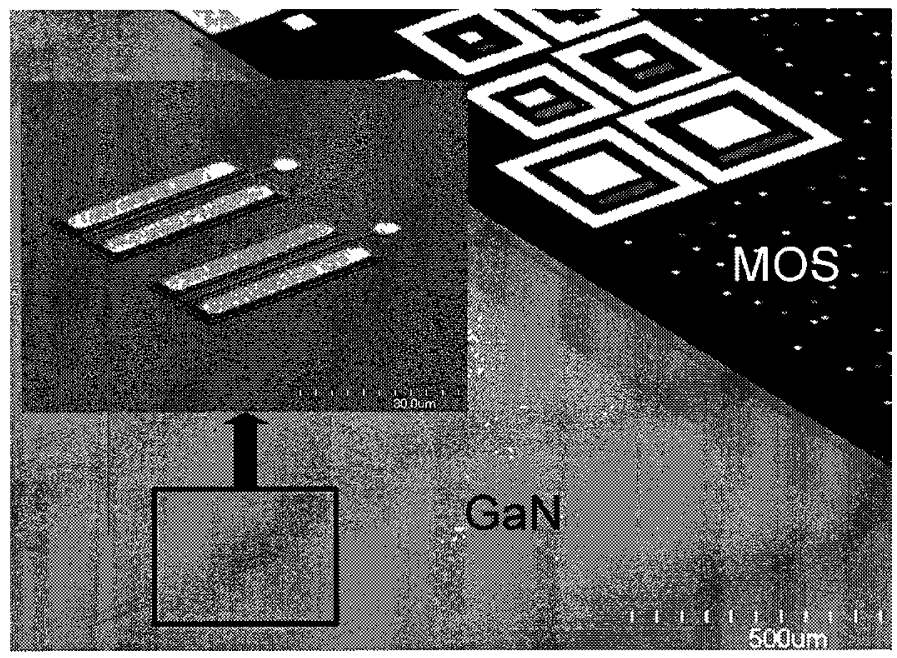

(a) Enclosed chip prior to mettalization

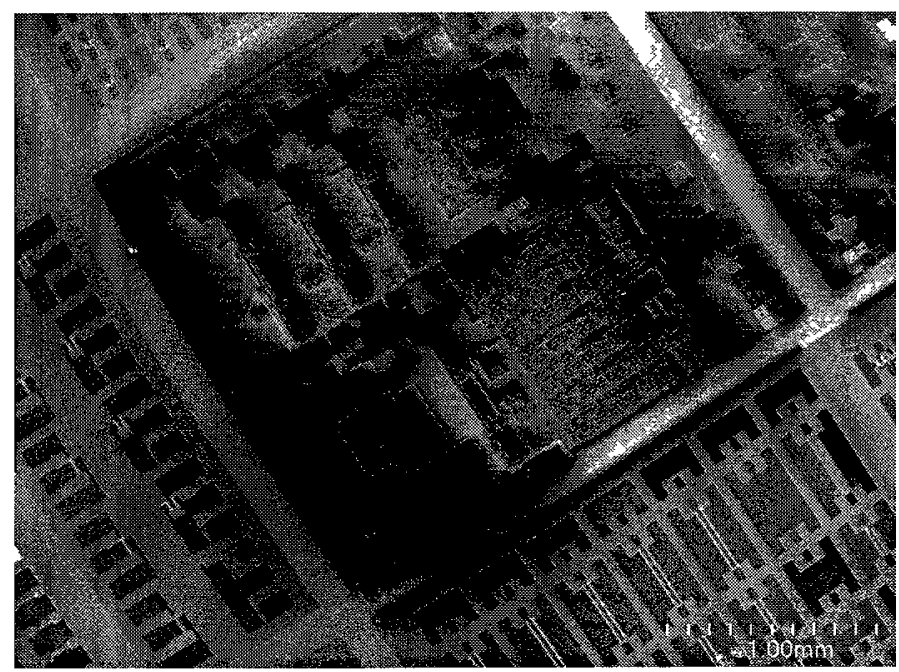

(b) Full chip after final fabrication

Figure 5.13: SEM images of integrated GaN HFET and MOS devices 


\subsection{Summary}

The first iteration of a successful process was able to generate good quality GaN transistors in conjunction with enclosed MOS devices. The MOS devices were shown to have gate and drain leakage due to a poor quality oxide and deep diffused junctions. A second fabrication round was conducted, which attempted to solve the gate leakage and drain leakage issues with the MOS process, as well as produce isolated devices. The performance of both the MOS and GaN devices is presented in the following chapters. 


\section{Chapter 6}

\section{MOS Device Performance}

The performance of the MOS devices fabricated in the joint GaN/CMOS process is presented in this chapter. Measurements from the first set of enclosed devices were used to design a second iteration of the process, which was presented in Section 5.6. DC-IV curves as well as CV analysis were used to examine the gate oxide quality of various wafers. The results from the second iteration of the joint run are shown. Possible improvements to the process based on these results are presented in the summary.

\subsection{First Run of Enclosed MOS Devices}

The performance of the first run of enclosed MOS devices (batch 464L) is reported here. These devices were successfully fabricated, but had two performance issues that needed to be resolved. The DC characteristics for an enclosed MOS device are shown in Figure 6.1.

A subthreshold swing of $70.4 \mathrm{mV} /$ decade was measured, demonstrating strong gate control of channel charge. The drain leakage, however, was higher than expected and the gate leakage was very high. The drain leakage can be explained by the shallow P-Well implant and deeper diffused junctions. We suspected the gate leakage to be 
the result of the low temperature of the oxidation in combination with orientation of the silicon. However, breakdown voltage and gate leakage measurements presented in Table 6.3 show that our low temperature oxidation can yield high quality oxides. Additionally, a fabrication error was made during the pre-oxidation clean which resulted in some minor surface etching of the silicon; this could also have been a contributing factor to the high leakage current in the gate oxide.

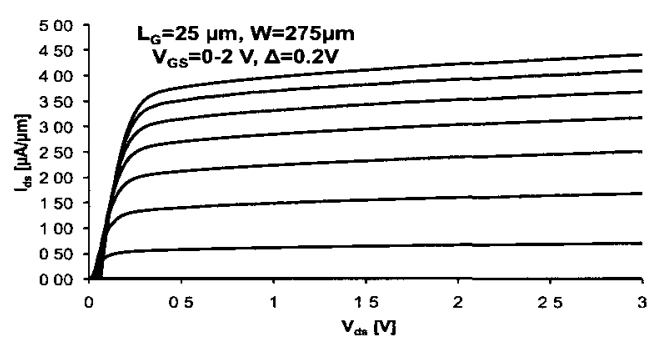

(a) Forward current: $I_{D S}$ vs. $V_{D S}$

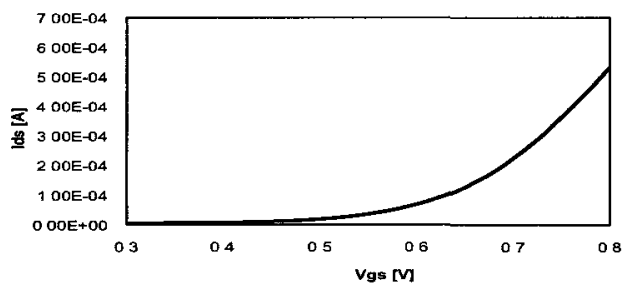

(c) Threshold voltage: $I_{D S}$ vs. $V_{G S}$

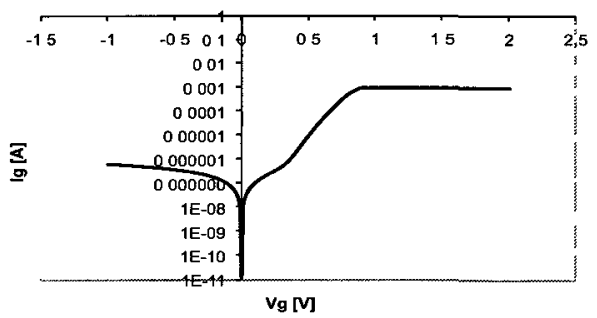

(e) Gate leakage: $\mathrm{I}_{G S}$ vs. $\mathrm{V}_{G S}$

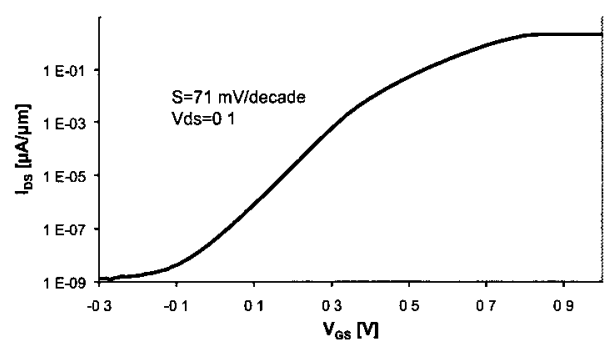

(b) Subthreshold: $I_{D S}$ vs. $V_{G S}$

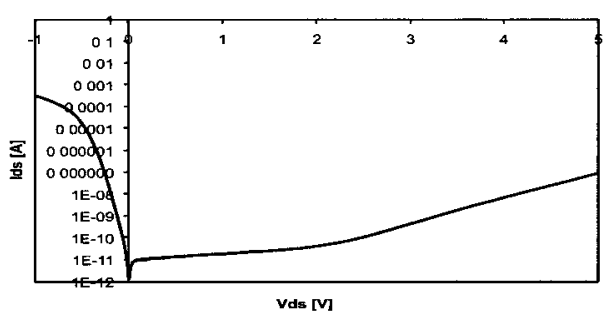

(d) Drain leakage: $I_{D S}$ vs. $V_{D S}$

Figure 6.1: $D C$ characteristics of enclosed NMOS device ( $\mathrm{W}=275 \mu \mathrm{m}, \mathrm{L}=25 \mu \mathrm{m})$ on sample 464LA 


\begin{tabular}{|c||c|c|c|}
\hline Device & Vt Calculated & Vt TSuprem4 & Vt Actual \\
\hline 464LC & 0.31 & 0.35 & 0.36 \\
464LA & 0.45 & 0.55 & 0.64 \\
\hline
\end{tabular}

Table 6.1: Threshold voltage comparison for batch 464L

The threshold voltages of the samples probed during the initial run are summarized in Table 6.1. In the case of the 464LC run, the threshold was quite close to that predicted by Tsuprem4 simulations as well as hand calculations using standard formulas for threshold voltage [75]. Sample 464LA however, had a higher threshold voltage than $464 \mathrm{LC}$, which is most likely explained by the surface concentration of the P-Well implant. Additionally, MOS capacitors were measured on test samples produced in earlier runs using CV analysis, as described in Section 6.3. The threshold voltage, which was extracted using the procedure described in Appendix D, was also found to vary from the predicted value. As the thermal budget of the process was altered to reduce the risk of GaN decomposition, the diffusion of the P-Well was also reduced, resulting in a higher concentration at the surface and hence a higher threshold voltage. This also had an impact on the drain leakage as the combination of a deep junction and a shallow $\mathrm{P}$-well can lead to increased leakage. Finally, the background doping of the substrate was changed. Initially, the process was designed for high-resistivity P-type silicon substrates, with a doping of around $10^{13} \mathrm{~atm} / \mathrm{cm}^{3}$. However, the growth wafers were switched to intrinsic silicon after the process was designed. This variation in background doping also had an impact on the drain leakage as the $\mathrm{n}+$ junctions are not guaranteed to be fully surrounded by P-type silicon due to the shallow nature of the well implant. 


\subsection{Second Process Iteration; Higher Tempera- ture Oxidations, Isolated Devices}

As described in Section 5.6, a second set of devices was fabricated in which higher temperature oxidations were used. Additionally, a new device isolation scheme was attempted. Fully functioning integrated samples were achieved in batches 484L, 487L and 488L. 484L and 487L were enclosed devices, in which the gate oxidation was performed at $900^{\circ} \mathrm{C}$ and $950^{\circ} \mathrm{C}$, respectively. Batch $488 \mathrm{~L}$ used the full MOS layout using a thick deposited oxide for field isolation. The gate oxidation was performed at $900^{\circ} \mathrm{C}$. Figure 6.2 shows the DC characteristics for a $2.5 \mu \mathrm{m}$ long test device for sample 488LA.

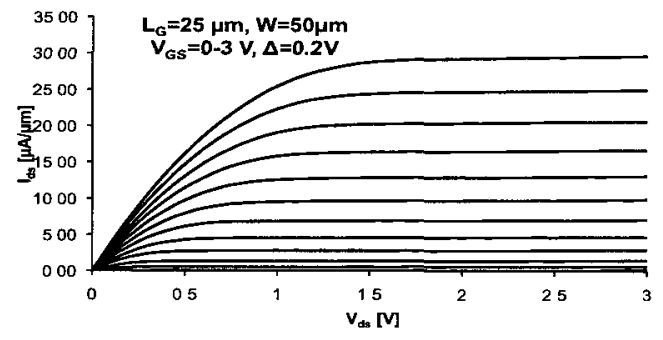

(a) Forward current: $I_{D S}$ vs. $V_{D S}$

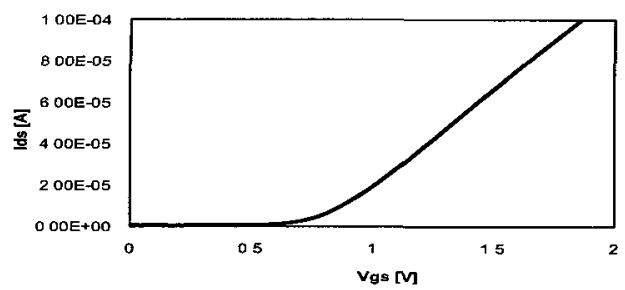

(c) Threshold Voltage: $I_{D S}$ vs. $V_{G S}$

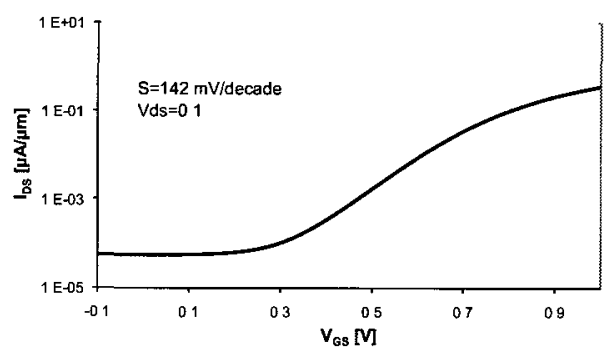

(b) Subthreshold: $\mathrm{I}_{D S}$ vs. $\mathrm{V}_{G S}$

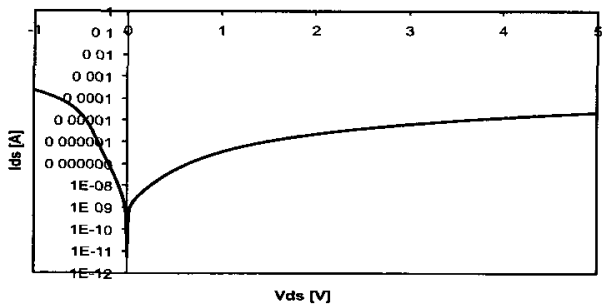

(d) Drain Leakage: $I_{D S}$ vs. $V_{D S}$

Figure 6.2: DC characteristics of Isolated NMOS device ( $\mathrm{W}=50 \mu \mathrm{m}, \mathrm{L}=2.5 \mu \mathrm{m})$ for sample 488LA

For sample 488LA, the gate leakage is not shown as it was lower than $0.1 \mathrm{pA}$ for 


\begin{tabular}{|c||c|c|c|c|c|c|}
\hline Sample & Orientation & Isolation & $\mathrm{V}_{T}$ & Gate Leakage & Drain Leakage & Subthreshold Swing \\
\hline 464LCB & 111 & Enclosed & $0.35 \mathrm{~V}$ & Yes & Yes & $79 \mathrm{mV} / \mathrm{dec}$ \\
464LA & 111 & Enclosed & $0.64 \mathrm{~V}$ & Yes & Yes & $78 \mathrm{mV} / \mathrm{dec}$ \\
$484 \mathrm{LC}$ & 110 & Enclosed & $0.29 \mathrm{~V}$ & No & No & $96 \mathrm{mV} / \mathrm{dec}$ \\
$487 \mathrm{LA}$ & 111 & Enclosed & $-0.06 \mathrm{~V}$ & Yes & Yes & NA \\
$487 \mathrm{LB}$ & 110 & Enclosed & $0.39 \mathrm{~V}$ & No & No & $90 \mathrm{mV} / \mathrm{dec}$ \\
$488 \mathrm{LA}$ & 111 & Yes & $0.83 \mathrm{~V}$ & No & Yes & $142 \mathrm{mV} / \mathrm{dec}$ \\
$488 \mathrm{LD}$ & 110 & Yes & $0.74 \mathrm{~V}$ & No & Yes & $148 \mathrm{mV} / \mathrm{dec}$ \\
\hline
\end{tabular}

Table 6.2: CMOS device summary. $12.5 \mu \mathrm{m}$ and $2.5 \mu \mathrm{m}$ gate length data given

an area of $750 \mu \mathrm{m}^{2}$, which is below the $4155 \mathrm{c}$ noise floor. The gate voltage was swept to a bias of $10 \mathrm{~V}$ in this case. This suggests that gate leakage had been eliminated for this batch. There was still drain leakage in these devices, as well as a very poor subthreshold swing.

Table 6.2 summarizes the DC characteristics of all the successful integrated GaN/MOS samples. Only the samples with the shortest gate length are reported here. The subthreshold swing of 487LA could not be measured due to high gate leakage currents.

There is a large variation in $V_{t}$ between batches, which is related to different oxide thicknesses as well as the difference in diffusion of the P-well implant, resulting in higher surface concentrations. The drain leakage issues were still not resolved for the second iteration of samples due to two factors: first, the well implant was performed through a thicker protective oxide than was desired (200 nm rather than $20 \mathrm{~nm}$ ) and second, the source/drain junction diffusion time was increased to 30 minutes in order to lower the source/drain sheet resistance. Because of this increased time however, the junction depth also increases. A solution to this problem would be to use implanted arsenic junctions, which would result in a much shallower profile. Figure 6.3 shows a TSuprem4 simulation of the P-Well implant with a $200 \mathrm{~nm}$ screen oxide used rather 
than a $20 \mathrm{~nm}$ screen oxide as desired. The lowest energy implant, which sets the threshold voltage is completely absorbed by the screen oxide. This explains the shallower P-well as well as the threshold voltage variability in the samples.

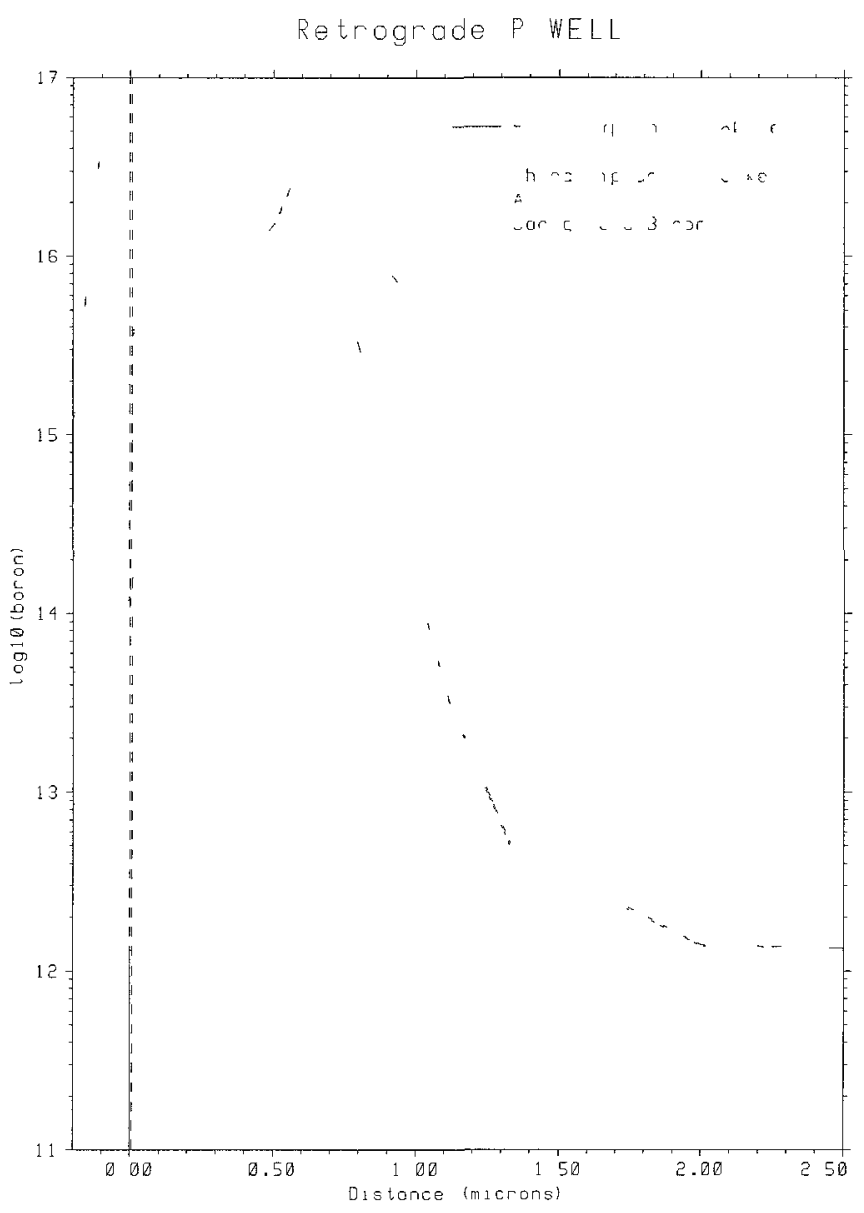

Figure 6.3: TSuprem4 simulation of P-Well implantation with $200 \mathrm{~nm}$ thick screen oxide used rather than $20 \mathrm{~nm}$

The primary goal of the MOS process redesign was to address the leakage in the gate oxide and attempt device isolation. The gate leakage was rectified for all batches except for $487 \mathrm{LA}$. The $484 \mathrm{~L}$ and $488 \mathrm{~L}$ batches were not expected to have a good quality oxide due to the lower oxidation temperature, however, both of these runs 
were successful. Additionally, batch 487 , in which the gate oxidation was performed at $950^{\circ} \mathrm{C}$, showed excellent oxide properties. The gate oxidation results are summarized in Section 6.3.

\subsubsection{Device Isolation in Batch $488 \mathrm{~L}$}

The method of using a thick deposited oxide for field isolation as described in Section 5.6 proved successful. Because test devices used to measure the parasitic field threshold voltage were left off the mask, this value could not be measured. Instead, the isolation between two adjacent wells was recorded. For a $2.5 \mu \mathrm{m}$ separation and $5 \mu \mathrm{m}$ separation between wells, the resistance was measured to be $291 k \Omega$ and 454 $k \Omega$ for biases up to $5 \mathrm{~V}$. This resulted in several $\mu \mathrm{A}$ of current, which must be reduced for circuits to work properly. Once again, this was related to the deep drain and source diffusion, which could be easily rectified using implanted junctions. TSuprem4 simulations predict a parasitic field threshold of $9.4 \mathrm{~V}$

\subsection{Analysis of MOS Gate Dielectics}

Capacitance-Voltage analysis is used to determine the quality of gate oxide growth performed at $900^{\circ} \mathrm{C}$ and $950^{\circ} \mathrm{C}$ in a dry $\mathrm{O}_{2}$ environment. As initial enclosed devices showed higher than average gate leakage, this tool was important to determine the minimum temperature needed for a good quality oxide, while not causing deterioration of the GaN layers via thermal decomposition.

The test setup included an HP4280 C-V meter controlled by HP VEE software through a GPIB interface. A standard probe station was used to contact the devices.

Figure 6.4 shows the CV data for three oxide test wafers. These wafers are patterned with aluminum dots on top and coated with aluminum on the backside for contacts during measurement. 


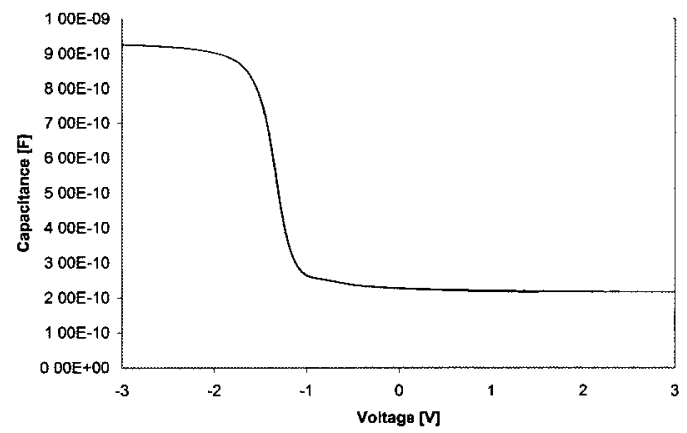

(a) $950^{\circ} \mathrm{C}$ test $(458 \mathrm{~L})$

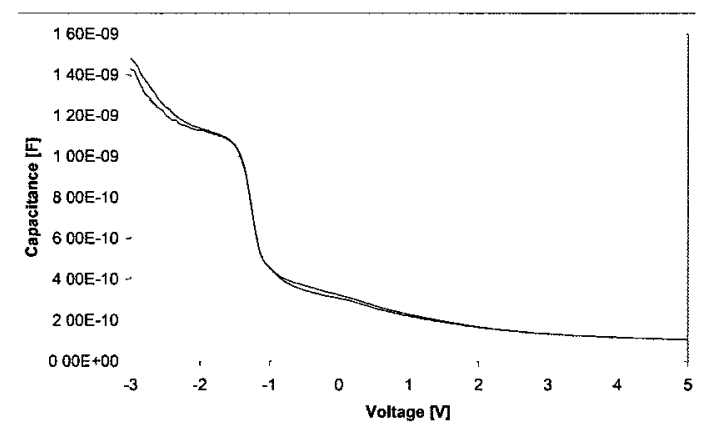

(b) $900^{\circ} \mathrm{C}$ test (etched)(464L)

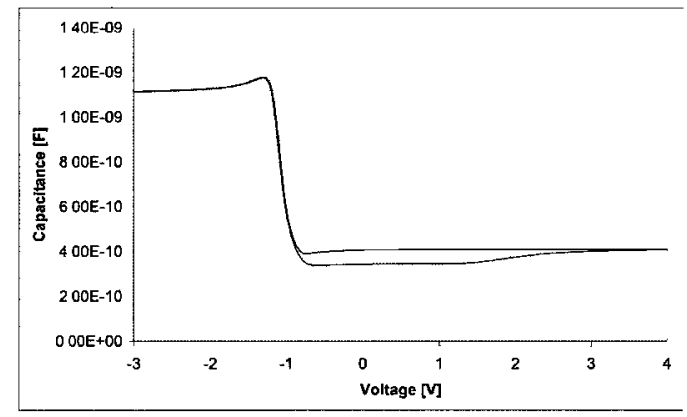

(c) $900^{\circ} \mathrm{C}$ test $(484 \mathrm{~L})$

Figure 6.4: CV curves for gate oxides on test wafers using CV dots

The first devices processed that included a $950^{\circ} \mathrm{C}$ gate oxidation were unsuccessful due to GaN decomposition at the elevated temperatures needed for the field oxidation. However, the MOS devices displayed zero gate leakage, which is an indication of a good quality oxide layer. The CV curve of the gate oxidation used for these devices is shown in Figure 6.4a. The flat curve in the accumulation region of the plot along with the sharp transition are indicative of a good oxide. The curve also shows no hysteresis, demonstrating a lack of mobile ion charge in the oxide.

Figure 6.4b shows the CV curve for the first working integrated MOS chip. In this case, a $900^{\circ} \mathrm{C}$ oxidation was used for the enclosed NMOS devices that demonstrated extremely high gate leakage. Unlike Figure 6.4a, this curve shows a sloped characteristic in accumulation, which is indicative of a leakage current in the oxide. During the processing of this sample, the silicon surface was accidentally etched directly prior to 


\begin{tabular}{|c|c|c|c|c|c|c|}
\hline Batch & Orientation & Oxidation Temp & Thickness & Oxidation Time & Leakage & Breakdown \\
\hline $464 \mathrm{~L}$ & 111 & $900^{\circ} \mathrm{C}$ & $315 \AA$ & $33 \mathrm{~min}$ & Yes & $3.9 \mathrm{MV} / \mathrm{cm}$ \\
$484 \mathrm{~L}$ & 111 & $900^{\circ} \mathrm{C}$ & $177 \AA$ & $23 \mathrm{~min}$ & Yes & $8.4 \mathrm{MV} / \mathrm{cm}$ \\
$484 \mathrm{~L}$ & 100 & $900^{\circ} \mathrm{C}$ & $110 \AA$ & $23 \mathrm{~min}$ & Yes & $9.6 \mathrm{MV} / \mathrm{cm}$ \\
$485 \mathrm{~L}$ & 111 & $950^{\circ} \mathrm{C}$ & $226 \AA$ & $14 \mathrm{~min}$ & No & $9.1 \mathrm{MV} / \mathrm{cm}$ \\
$485 \mathrm{~L}$ & 110 & $950^{\circ} \mathrm{C}$ & $223 \AA$ & $14 \mathrm{~min}$ & No & $12.4 \mathrm{MV} / \mathrm{cm}$ \\
$487 \mathrm{~L}$ & 111 & $950^{\circ} \mathrm{C}$ & $285 \AA$ & $19 \mathrm{~min}$ & No & $7.9 \mathrm{MV} / \mathrm{cm}$ \\
$487 \mathrm{~L}$ & 100 & $950^{\circ} \mathrm{C}$ & $187 \AA$ & $19 \mathrm{~min}$ & No & $8.7 \mathrm{MV} / \mathrm{cm}$ \\
$488 \mathrm{~L}$ & 111 & $900^{\circ} \mathrm{C}$ & $175 \AA$ & $25 \mathrm{~min}$ & No & $12.6 \mathrm{MV} / \mathrm{cm}$ \\
$488 \mathrm{~L}$ & 110 & $900^{\circ} \mathrm{C}$ & $164 \AA$ & $25 \mathrm{~min}$ & No & $10.7 \mathrm{MV} / \mathrm{cm}$ \\
\hline
\end{tabular}

Table 6.3: Oxidation thickness summary using test wafers for each batch.

gate oxidation, which resulted in some surface roughness. This is a possible explanation for the poor oxide quality. Additionally, the lower oxidation temperature and crystal orientation can also be used to explain the low quality film [76] [77].

Finally, Figure 6.4c shows the CV curve for a secondary $900^{\circ} \mathrm{C}$ oxidation test in which the silicon was properly processed prior to oxidation. This curve shows a high quality oxide compared to that of Figure 6.4b, suggesting that the rough silicon surface used for the MOS processing may have been a major cause of the gate leakage.

It should be noted that batch 485L did not yield any successful GaN/MOS integrated devices. However, the oxides, which were grown at $950^{\circ} \mathrm{C}$, were still analyzed.

\subsection{Oxide Breakdown Measurements}

Oxide breakdown was determined by measuring the current through the oxide using two successive voltage sweeps. A good quality oxide will reproduce the same curve as the bias is increased, whereas a poor oxide will show hysteresis. The hysteresis is caused by the presence of defects in the oxide layer that result in charge trapping, which shifts in the gate current voltage characteristic. The second sweep is continued 
to a higher voltage until destructive breakdown is reached. The breakdown field is subsequently calculated by dividing this voltage by the oxide thickness. These values are summarized in Table 6.3. The second batch of samples all show excellent oxide quality, with the breakdown field reaching up to $12.6 \mathrm{MV} / \mathrm{cm}$, which is indicative of a good quality oxide. Figure 6.5 shows the oxide test curves for two CV test structures on batches $485 \mathrm{~L}$ and $488 \mathrm{~L}$.

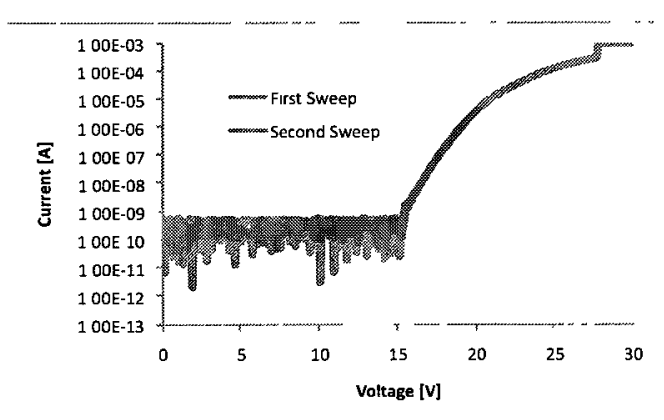

(a) $485 \mathrm{~L} 950^{\circ} \mathrm{C}$ test oxide

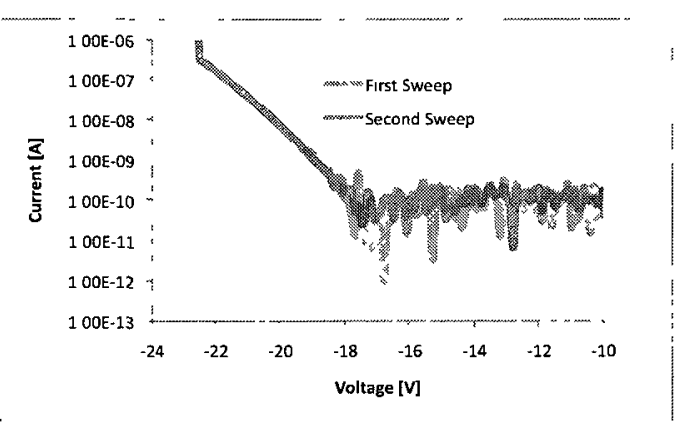

(b) $488 \mathrm{~L} 900^{\circ} \mathrm{C}$ test oxide

Figure 6.5: Oxide breakdown curves for test oxides for batch 485L and 488L $(<111>$ oriented)

The oxides in the first run of samples (batch $464 \mathrm{~L}$ ) broke down at $3.9 \mathrm{MV} / \mathrm{cm}$. This low breakdown was expected, as the quality of the oxide was known to be poor. Additionally, the pad oxide of only $10 \mathrm{~nm}$ used for the LOCOS technique employed in batch $485 \mathrm{~L}$ had a dielectric breakdown of $9.8 \mathrm{MV} / \mathrm{cm}$. This demonstrates that the oxide quality is still very good at lower thicknesses.

\subsection{Summary}

Two iterations of integrated GaN/MOS samples were successfully completed and devices tested. The first run, which only included enclosed MOS devices, demonstrated good forward current and subthreshold performance, but was plagued with high gate 
leakage as well as drain leakage. The second iteration of MOS runs attempted oxidations at slightly higher temperatures, as well as a new isolation scheme based on a thick deposited oxide. An attempt to use LOCOS isolation failed once again due to GaN decomposition. The gate oxide quality of the second run of samples was excellent, even for the oxidations performed at $900^{\circ} \mathrm{C}$. This suggests that the gate leakage demonstrated by the first batch of MOS samples was due to process error rather than low oxidation temperature.

The second iteration of devices still showed junction leakage issues, which were caused by a shallow P-well implantation and over-diffusion of the junctions. This can be easily rectified by using implanted junctions for future processes. The isolation scheme using a thick CVD oxide worked successfully, allowing for $2.5 \mu \mathrm{m}$ gate length devices to be successfully fabricated. Although small leakage current was measured between adjacent wells, this technique showed promise in allowing for circuits to be implemented in the process without compromising the thermal budget.

The MOS device performance did not show variation with the orientation of the substrate. The oxide thickness for both orientations was approximately the same, as was the oxide quality. Ideally, the $<110>$ orientation is preferred due to the increased hole mobility and relevance to state-of-the-art technology.

Three improvements to the existing process must be made. Firstly, the junction depth must be reduced in order to mitigate short-channel effects and junction leakage. Secondly, the source of the threshold voltage variation must be determined and rectified. This is most likely caused by the diffusion of the P-well being different for each run as the thermal budget is different. Finally, the device isolation scheme must be improved to eliminate all leakage currents. This is related to the deep junctions and variation in substrate doping, both factors that can be adjusted. 


\section{Chapter 7}

\section{GaN Device Performance}

The performance of the GaN HEMT devices is reported in this chapter. Both fieldplated and non-field-plated dual-gate HEMTs were fabricated, with gate widths ranging from $10 \mu \mathrm{m}$ to $320 \mu \mathrm{m}$ and a fixed gate length of $0.8 \mu \mathrm{m}$. A summary of the DC characteristics from all wafers that successfully completed fabrication is presented in this chapter. The breakdown voltage of the devices, gate leakage, and current collapse are also investigated. Suggestions for improvement are given in the summary.

\section{$7.1 \quad$ Measurement Summary}

Seven samples in total from quarters of two-inch wafers on which GaN was grown were successfully fabricated with both MOS and GaN devices; two samples (464L batch) were part of the first batch of experiments, while the remaining five were fabricated in the second iteration of the design as described in Chapter 5 . The performance of HEMTs from all the successful wafers is summarized in Table 7.1.

All DC measurements were made using a standard low-frequency probe station setup that included four micro-manipulators with tungsten probes connected to an Agilent 5155c semiconductor parameter analyzer. TLM structures were used to measure sheet resistance and the specific contact resistances of the $\mathrm{Ti} / \mathrm{Al} / \mathrm{Ti} / \mathrm{Al}$ ohmic 


\begin{tabular}{|c||c|c|c|c|c|c|c|}
\hline Wafer & Si Type & C-doped & Max $\mathrm{I}_{D S}$ & $\mathrm{~V}_{\text {Panchoff }}$ & Gate Leakage & Peak $\mathrm{g}_{m}$ & $\mathrm{R}_{c}$ \\
\hline 464LCB & 111 & Yes & $0.45 \mathrm{~A} / \mathrm{mm}$ & $-4.7 \mathrm{~V}$ & $1.2 \times 10^{-2} \mathrm{~A} / \mathrm{mm}$ & $\mathrm{x}$ & $\mathrm{x}$ \\
464LA & 111 & No & $0.81 \mathrm{~A} / \mathrm{mm}$ & $-3.3 \mathrm{~V}$ & $7.2 \times 10^{-3} \mathrm{~A} / \mathrm{mm}$ & $181 \mathrm{mS} / \mathrm{mm}$ & $1.03 \times 10^{-4} \Omega \mathrm{cm}^{2}$ \\
$484 \mathrm{LC}$ & 110 & No & $0.82 \mathrm{~A} / \mathrm{mm}$ & $-3.4 \mathrm{~V}$ & $4.4 \times 10^{-3} \mathrm{~A} / \mathrm{mm}$ & $191 \mathrm{mS} / \mathrm{mm}$ & $1.65 \times 10^{-3} \Omega \mathrm{cm}^{2}$ \\
$484 \mathrm{LA}$ & 111 & No & $0.63 \mathrm{~A} / \mathrm{mm}$ & $-2.3 \mathrm{~V}$ & $3.2 \times 10^{-2} \mathrm{~A} / \mathrm{mm}$ & $146 \mathrm{mS} / \mathrm{mm}$ & $4.45 \times 10^{-4} \Omega \mathrm{cm}^{2}$ \\
487LB & 110 & No & $0.31 \mathrm{~A} / \mathrm{mm}$ & $-3.6 \mathrm{~V}$ & $3.2 \times 10^{-2} \mathrm{~A} / \mathrm{mm}$ & $47 \mathrm{mS} / \mathrm{mm}$ & $2.32 \times 10^{-4} \Omega \mathrm{cm}^{2}$ \\
$488 \mathrm{LA}$ & 111 & No & $0.72 \mathrm{~A} / \mathrm{mm}$ & $-2.7 \mathrm{~V}$ & $8.7 \times 10^{-3} \mathrm{~A} / \mathrm{mm}$ & $165 \mathrm{mS} / \mathrm{mm}$ & $3.03 \times 10^{-4} \Omega \mathrm{cm}^{2}$ \\
$488 \mathrm{LD}$ & 110 & No & $0.67 \mathrm{~A} / \mathrm{mm}$ & $-3.6 \mathrm{~V}$ & $1.58 \times 10^{-2} \mathrm{~A} / \mathrm{mm}$ & $126 \mathrm{mS} / \mathrm{mm}$ & $8.22 \times 10^{-3} \Omega \mathrm{cm}^{2}$ \\
\hline
\end{tabular}

Table 7.1: Summary of GaN device performance for successful runs. X denotes a value that could not be measured.

contacts. Figure 7.1 summarizes the DC performance of a typical dual-gate GaN HEMT device with a gate length of $0.8 \mu \mathrm{m}$ and total width of $80 \mu \mathrm{m}$.

The device performance varied from sample to sample, with the best samples conducting over $0.8 \mathrm{~A} / \mathrm{mm}$ of forward current for maximum usable gate bias of $3 \mathrm{~V}$. This compares quite well to state-of-the-art devices on silicon substrates which report $1.04 \mathrm{~A} / \mathrm{mm}$ forward current for $0.7 \mu \mathrm{m}$ long devices [7], and $0.6 \mu \mathrm{m}$ long devices on silicon-carbide that report $1.2 \mathrm{~A} / \mathrm{mm}$ [21]. The currents also scaled well with device width. Figure 7.2 shows the forward current vs. device width for sample 488LD. A constant current of $0.7 \mathrm{~A} / \mathrm{mm}$ is maintained. The field-plated had the same DC characteristics as the non field-plated devices.

Wafers that did not have as much forward current typically had worse underlying electrical properties, such as sheet resistance and mobility. It was disappointing, however, that the ohmic contact scheme, which worked well on sample 464LA, did not work as well for the next set of samples, limiting the forward current. This is especially the case for sample 484LC, which still had the best forward current even though it was plagued with high contact resistance. A performance difference between the devices fabricated on $\langle 111\rangle$ oriented and $<110\rangle$ oriented substrates 


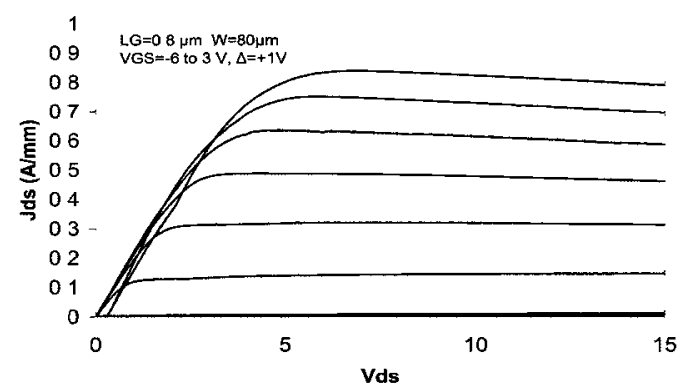

(a) Forward current: $I_{D S}$ vs. $V_{D S}$

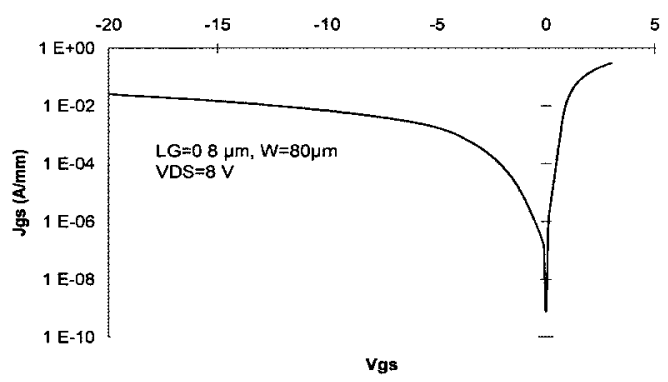

(c) Gate Leakage: $\mathrm{J}_{G S}$ vs $\mathrm{V}_{G S}$

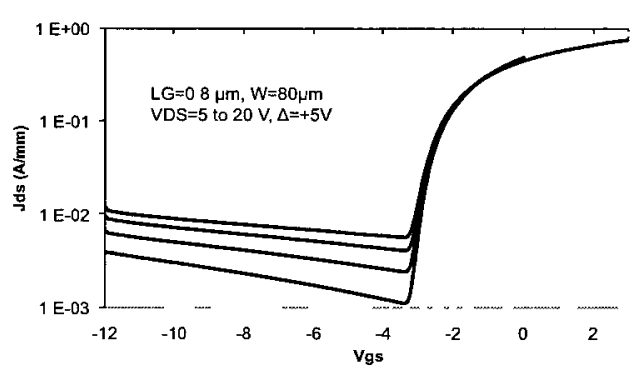

(b) Pinch Off: $\mathrm{J}_{G S}$ vs $\mathrm{V}_{G S}$

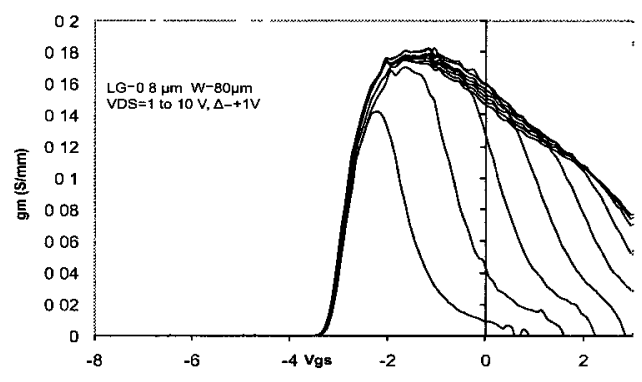

(d) Transconductance: $\mathrm{g}_{m}$ vs $\mathrm{V}_{G S}$

Figure 7.1: DC measurements for dual-gate AlGaN/GaN HEMT on sample 464LA was not observed.

\subsection{Gate Leakage}

The amount of gate leakage in all devices fabricated is summarized in Table 7.1. The values ranged from $4.4 \times 10^{-3} \mathrm{~A} / \mathrm{mm}$ for the best wafer to $3.2 \times 10^{-2} \mathrm{~A} / \mathrm{mm}$. These values were taken at a bias of $-8 \mathrm{~V}$ on the gate and $-10 \mathrm{~V}$ on the drain. It should be noted that the pinchoff and gate leakage characteristics are similar for all samples except for the C-doped wafer, 464LC. All non C-doped samples showed the same trend in gate leakage: a rising leakage current as the gate becomes highly reverse biased. However, the C-doped sample had a flat leakage curve as shown by the dashed line in Figure 7.3, which compares the gate leakage for a C-doped (464LC) vs. non Cdoped (464LA) sample. This can be explained by the fact that the contribution of 


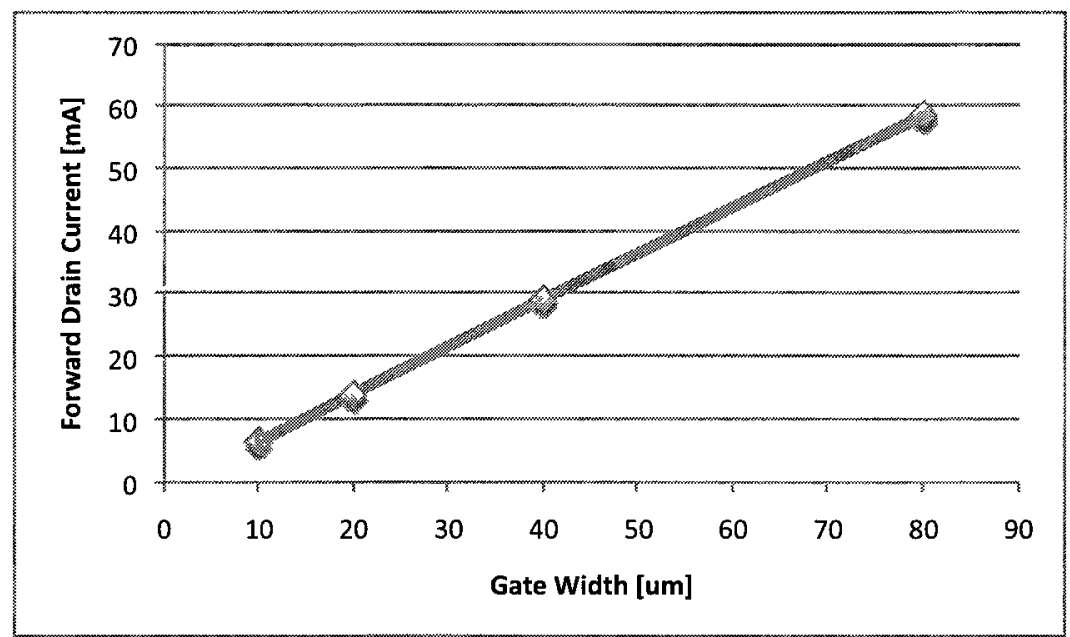

Figure 7.2: Maximum forward current vs. device width for sample 488LA

leakage current through the buffer layer is eliminated only in the case of the C-doped sample. In non C-doped samples substrate leakage increases as the gate-drain voltage is increased.

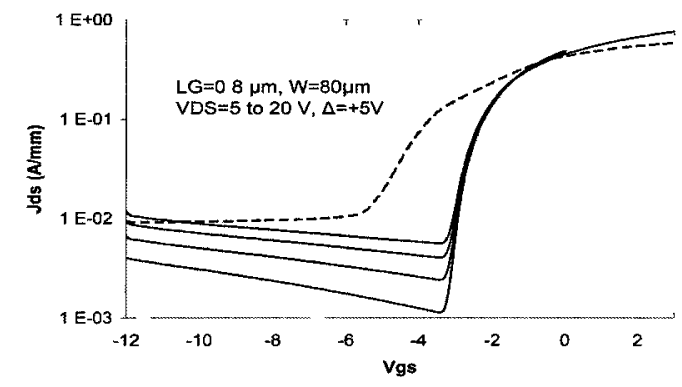

(a) Forward current: $I_{D S}$ vs. $V_{D S}$

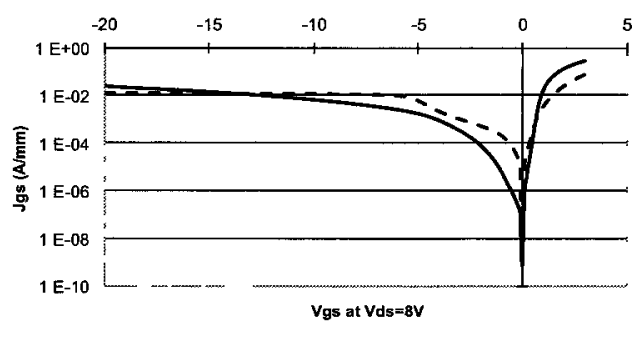

(b) Pinch-off: $\mathrm{J}_{G S}$ vs $\mathrm{V}_{G S}$

Figure 7.3: Gate leakage comparison for C-doped 464LC sample (dashed) vs non C-doped 464LA sample

The magnitude of gate leakage for all the devices remained high. This was primarily due to the fact that the passivation layer, which was needed to eliminate current collapse, had not been optimized to mitigate gate leakage nor for growth on silicon substrates. Increasing gate leakage after passivation is a common issue for all GaN 
HEMT structures, irrespective of substrate. Techniques such as aluminum oxide passivations and nitrogen plasma treatments have been used to reduce the effects of gate leakage [78].

\subsection{Current Collapse}

Pulsed measurements have shown that the nitride passivation layer had mitigated current collapse for all wafers. Figure 7.4 shows both the swept and pulsed DC drain characteristics. The pulsed characteristic was obtained by maintaining the gate terminal at a baseline voltage of $-8 \mathrm{~V}$ to fill surface traps. The gate was then pulsed with a $0.5 \mathrm{~ms}$ pulse with a period of $10 \mathrm{~ms}$ to the desired bias point for the measurement. The current drive remained the same for both conditions, showing that current collapse was not an issue for this device. This was the case for all the successfully fabricated wafers.

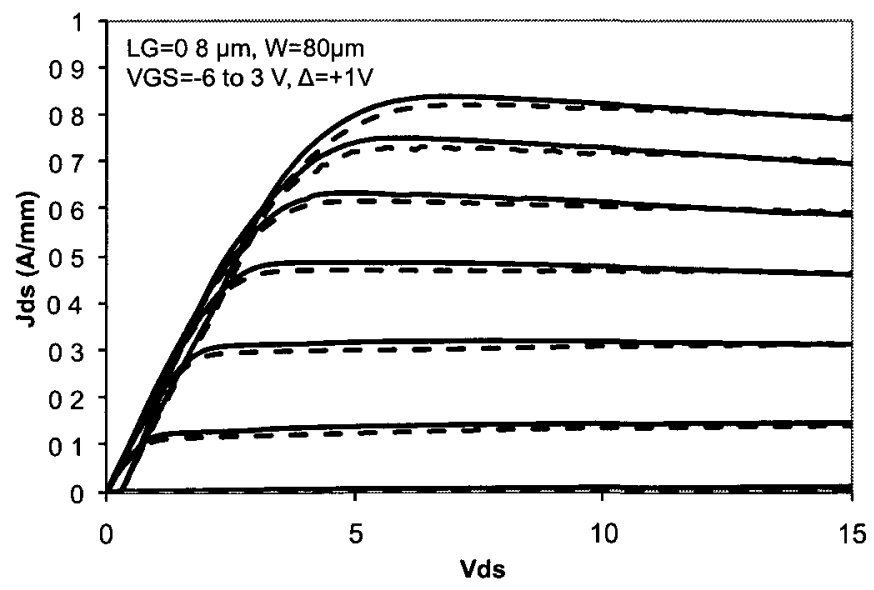

Figure 7.4: Pulsed (dashed) and non-pulsed (solid) $\mathrm{J}_{D S}$ vs $\mathrm{V}_{D S}$ curves for wafer 464LA 
Table 7.2: Breakdown Voltage vs Gate-Drain Spacing

\begin{tabular}{|c|c|}
\hline Gate-Drain Spacing & Breakdown Voltage \\
\hline $2.2 \mu \mathrm{m}$ & $54 \mathrm{~V}$ \\
$3.2 \mu \mathrm{m}$ & $93 \mathrm{~V}$ \\
$4.2 \mu \mathrm{m}$ & $140 \mathrm{~V}$ \\
$5.2 \mu \mathrm{m}$ & $>200 \mathrm{~V}$ \\
\hline
\end{tabular}

\subsection{Breakdown Characteristics}

Breakdown measurements were performed on the best sample, 464LA. The breakdown voltage of the devices ranged from $53 \mathrm{~V}$ for a $2.2 \mu \mathrm{m}$ gate-drain spacing to greater than $200 \mathrm{~V}$ for a $5.2 \mu \mathrm{m}$ gate-drain spacing. It should be noted that $200 \mathrm{~V}$ was the measurement limit of the equipment in this case. To make this measurement, devices were pinched off with a gate voltage of $-8 \mathrm{~V}$ while the drain voltage was increased. Breakdown was observed by monitoring the gate current, as this increased sharply at the onset of breakdown. Table 7.2 demonstrates this trend correlating the gate-drain spacing to the breakdown voltage as shown in [79]. These high breakdown voltages on non-optimized devices demonstrate the potential of HEMT integration to greatly increase the voltage-handling capability of MOS.

\subsection{Summary}

GaN HEMT devices ranging from $2 \times 5 \mu \mathrm{m}$ to $2 \times 160 \mu \mathrm{m}$ wide with a gate length of $0.8 \mu \mathrm{m}$ were successfully fabricated and tested on $<111>$ and $<110>$ oriented silicon substrates. The GaN devices fabricated in this work showed comparable performance to state-of-the-art technology in terms of forward current, and show no signs of current collapse. However, gate leakage and a non-reproducible ohmic contact scheme are two issues that must be addressed. The breakdown characteristic also looks promising for 
high-power and/or high-voltage applications. There were no major differences in the performance between the two types of substrate used.

Future work to improve the performance of the GaN devices should include field plates, as these have become standard practice to improve the breakdown characteristic and hence, allow operation at higher voltage and power levels. Additionally, the ohmic contact scheme must be revisited. Although the $\mathrm{Ti} / \mathrm{Al} / \mathrm{Ti} / \mathrm{Al}$ scheme did provide some promising results, it was shown to be less reproducible and is not comparable performance-wise to gold-based ohmic schemes that are currently in use. Experiments should be performed to determine whether gold in the ohmic contact scheme can be kept isolated from the MOS devices. Additionally, further investigation into silver and vanadium-based contacts could be beneficial. Finally, the gate leakage of these devices must be reduced. Optimized field plates can both increase breakdown voltage and reduce gate leakage. 


\section{Chapter 8}

\section{Conclusions and Future Work}

The first monolithic integration of silicon MOSFETs and GaN HEMT devices has been presented in this work. A co-integration technique that minimizes thermal budget in order to prevent GaN decomposition has been developed. This is based on a novel windowed epitaxy technique that was presented in Chapter 4 , that allows for high-quality GaN layers to be grown on both $<111>$ and $<110>$ oriented silicon while preserving an atomically smooth silicon surface for MOS fabrication. The process flows presented in Chapter 5 yielded isolated MOS devices with good quality gate oxides alongside GaN HEMTs which show high breakdown characteristics, comparable forward current to state-of-the-art devices and no current collapse. The MOSFET and GaN HEMT device performance was summarized in Chapters 6 and 7.

\subsection{Future Directions}

The primary focus for the continuation of the work is to bring the performance of the MOS and GaN devices up to state-of-the art levels. For the MOS devices, the process needs to be optimized to eliminate all short-channel effects and drain leakage issues, which were mostly related to the depth of the source/drain diffusions, but not limited to these factors. The isolation scheme based on a thick deposited oxide shows promise 
to be sufficient for $2.5 \mu \mathrm{m}$ long devices, but not for current sub-micron sized technology. Good quality oxides were grown at both $900^{\circ} \mathrm{C}$ and $950^{\circ} \mathrm{C}$ which is not only important to reduce GaN decomposition, but also to minimize unwanted diffusion of source/drain junctions as the technology is scaled. Demonstrating PMOS devices on $<110>$ oriented substrates is also a must for future research, as the increased hole mobility is the primary reason for pursuing this orientation.

The GaN devices in this work exceeded forward currents of $0.8 \mathrm{~A} / \mathrm{mm}$, which is comparable to current technology. The next steps to take in the GaN process development would be to reduce the gate leakage, which limits their application as power devices. The gate leakage can be reduced by optimizing the dielectric layer currently used to mitigate the effects of current collapse. Additionally, field plates, which have become widely used, need to be implemented and optimized to raise the breakdown voltage as well as reduce gate leakage current. Finally, the goldfree ohmic contact scheme developed for this work, which initially showed promising results, proved not to be reproducible, and hence, must be redeveloped.

With these process improvements the technology will be ready for circuit applications. Promising areas include power amplifier circuits with adaptive MOS-based circuitry, as well as GaN-based sensors that use MOSFETs for processing and readout [80]. GaN power-switching devices are also of great interest for routing and automotive applications. Having MOS readout and control circuitry alongside highbreakdown voltage GaN switches may be beneficial in this field.

\subsection{Summary of Contributions}

The first demonstration of the monolithic integration of silicon MOSFETs with GaN HEMT devices has been presented in this work. A new windowed-epitaxy technique was developed allowing for the growth of GaN HEMT layers alongside regions of 
atomically smooth silicon. A low thermal budget and gold-free fabrication process that forms isolated MOS devices ready to be used in circuit applications alongside high performing GaN HEMTs has been developed. This was accomplished on both $<111>$ and $<110>$ oriented silicon substrates. 


\section{List of References}

[1] S. Nuttick and E. Gebara, "Development of GaN Wide Bandgap Technology for Microwave Power Applications," IEEE Microwave Magazıne, pp. 80-86, March 2002.

[2] Y. Cordier and F. Semond, "MBE growth of AlGaN/GaN HEMTS on resistive $\mathrm{Si}(111)$ substrates with RF small signal and power performances," Journal of Crystal Growth, vol. 251, pp. 811-815, 2003.

[3] F. Schulze and A. Dadgar, "GaN heteroepitaxy on Si(001)," Appl. Phys. Lett., vol. 84, no. 4747, 2004.

[4] S. Joblot and F. Semond, "High-electron-mobility AlGaN/GaN heterostructures grown on $\mathrm{Si}(001)$ by molecular-beam epitaxy," Appl. Phys. Lett., vol. 87, no. $133505,2005$.

[5] Y. Cordier, J.-C. Moreno, and F. Semond, "Demonstration of AlGaN/GaN highelectron-mobility transistors grown by molecular beam epitaxy on $\operatorname{Si}(110)$," IEEE Electron Device letters, vol. 29, pp. 1187-1189, 2008.

[6] D. Dumka and C. Lee, "AlGaN/GaN HEMTs on Si substrate with $7 \mathrm{~W} / \mathrm{mm}$ output power density at $10 \mathrm{GHz}$," IEEE Electron Device Letters, vol. 25, no. 7, 2004.

[7] J. Johnson and E. Piner, "12 W/mm AlGaN-GaN HFETs on Silicon Substrates," IEEE Electron Device Letters, vol. 25, July 2004.

[8] "Process and device simulation tools." http://www. synopsys. com/Tools/TCAD/ Pages/default.aspx.

[9] U. Mishra and L. Shen, "GaN-Based RF Power Devices and Amplifiers," Proceedings of the IEEE, vol. 96, February 2008. 
[10] R. Borges, "Gallium Nitride electronic devices for high-power wireless applications," RF Semiconductors, pp. 72-82, September 2001.

[11] P. Bove and H. Lahreche, "Building Engineered Substrates for GaN HEMT applications," Solzd State Technology, pp. 43-48, January 2006.

[12] L. Liu and J. Edgar, "Substrates for Gallium Nitride Epitaxy," Materıals Scıence and Engineering R, vol. 37, pp. 61-127, April 2002.

[13] E. Chumbes and A. Schremer, "Microwave Performance of AlGaN/GaN High Electron Mobility Transistors on $\mathrm{Si}(111)$ Substrates," IEEE Electron Devices Meetıng. IEDM Technical Digest Internatıonal, pp. 397-400, December 1999.

[14] K. Marent, "Imec and aixtron set important step towards low-cost gan power devices," June 2008. IMEC Press Release.

[15] V. Hoel and N. Vellas, "High-power AlGaN/GaN HEMT on resistive silicon substrate," Electrons Letters, vol. 38, July 2002.

[16] R. Behtash and H. Tobler, "AlGaN/GAN HEMTs on $\mathrm{Si}(111)$ with $6.6 \mathrm{~W} / \mathrm{mm}$ output power density," Electronıcs Letters, vol. 39, April 2003.

[17] R. Therrien and S. Singhal, "A 36mm GaN-on-Si HFET Producing 368W at 60V with 70 percent Drain Efficiency," IEEE Electron Devices Meetıng. IEDM Technıcal Digest Internatıonal, pp. 568-571, December 2005.

[18] D. Ducatteau and E. Minko, "Output Power Density of $5.1 \mathrm{~W} / \mathrm{mm}$ at $18 \mathrm{GHz}$ with an AlGaN/GaN HEMT on Si substrate," IEEE Electron Device Letters, vol. 27, January 2006.

[19] D. Dumka and P. Saunier, "Gan on si hemt with $65 \%$ power added efficiency at 10 ghz," Electronics Letters, vol. 46, pp. 946-947, 242010.

[20] P. Srivastava, P. Das, and G. Borghs, "Silicon Substrate Removal of GaN DHFETs for Enhanced (¿1100 V) Breakdown Voltage," IEEE Electron Device Letters, vol. 31, no. 8, 2010.

[21] Y.-F. Wu, A. Saxler, and P. Parikh, "30 W/mm GaN HEMTs by Field-Plate Optimization," IEEE Electron Device Letters, vol. 25, no. 3, 2004.

[22] M. Yang, E. Gusev, M. Leong, and A. Chou, "Performance Dependence of CMOS on Silicon Substrate Orienatation for Ultrathin Oxynitride and $\mathrm{HfO}_{2}$ Gate Dielectrics," IEEE Electron Device Letters, vol. 24, May 2003. 
[23] H. Momose, T. Ohguro, K. Kojima, S. Nakamura, and Y. Toyshima, "1.5 nm Gate Oxide CMOS on (110) Surface-Oriented Si Substrate," IEEE Trans. on Electron Devices, vol. 50, April 2006.

[24] U. Mishra, P. Parikh, and Y.-F. Wu, "AlGaN-GaN HEMTs - An Overview of Device Operation and Applications ," Proceedıngs of the IEEE, vol. 90, June 2002.

[25] S. Pearton, C. Abernathy, and F. Ren, Gallum Nitride Processing for Electronıcs, Sensors and Spintronıcs. Springer-Verlag, 2006.

[26] J. Webb, H. Tang, S. Rolfe, and J. Bardwell, "Semi-insulating C-doped GaN and high-mobility AlGaN/GaN heterostructures grown by ammonia MBE," Applzed Physics Letters, vol. 75, August 1999.

[27] R. Vaudo, X. Xu, and G. Brandes, "Characteristics of Semi-insulating, Fe-doped GaN Substrates," Physıca Status Solıdı (A), vol. 200, no. 1, pp. 18-21, 2003.

[28] J. Ibbetson, P. Fini, and U. Mishra, "Polarization effects, surface states, and the source of electrons in AlGaN/GaN heterostructure field effect transistors," Applzed Physıcs Letters, vol. 77, no. 2, 2000.

[29] E. Yu and P. Asbeck, "Schottky barrier engineering in III-V nitrides via the piezoelectric effect," Applied Physıcs Letters, vol. 73, Sept 1998.

[30] R. Vetury, N. Zhang, S. Keller, and U. Mishra, "The Impact of Surface States on the DC and RF Characterisitics of AlGaN/GaN HFETs," IEEE Transactrons on Electron Devices, vol. 48, March 2001.

[31] A. Chini, D. Buttari, R. Coffie, and S. Heikman, "12 W/mm power density AlGaN-GaN HEMTs on sapphire substrate," IEEE Electron Device Letters, vol. 40, pp. 73-74, January 2004.

[32] E. Gusev, V. Narayanan, and M. Frank, "Advanced high-kappa dielectric stacks with polySi and metal gates: Recent progress and current challenges," IBM Journal of Research and Development, vol. 50, no. 4, pp. 387-410, 2006.

[33] F. Ootsuka, A. Katakami, and M. Tanjyo, "Ultralow-Thermal-Budget CMOS process Using Flash-Lamp Annealing for 45nm Metal/High-k FETs," IEEE trans. on Electron Devices, vol. 55, April 2008. 
[34] C. Ortolland, L. Ragnarsson, and T. Hoffman, "Optimized Ultra-Low Thermal Budget Process Flow for Advanced High-K/Metal Gate First CMOS Using LaserAnnealing Technology," 2009 Symposium on VLSI Technology, 2009.

[35] G. Lucovsky, M. Manatini, and E. Irene, "Low Temperature Silicon Dioxide Films: A study of Chemical Bonding by ellipsometry and infrared Spectroscopy," Journal of Vacuum Scrence and Technology, vol. 5, no. 2, 1987.

[36] E. EerNisse, "Stress in Thermal $\mathrm{SiO}_{2}$ during Growth," Applzed Physics Letters, vol. 35 , no. $1,1979$.

[37] H. Wendt, A. Spitzer, W. Bensch, and K. Sichart, "Improvement in Oxide Quality by Rapid Thermal Annealing," Journal of Applied Physıcs, vol. 67, June 1990 .

[38] M. Arienzo, L. Dori, and T. Szabo, "Effect of post-oxidation Anneal on Ultrathin $\mathrm{SiO}_{2}$ Gate Oxides," Applied Physics Letters, vol. 49, October 1986.

[39] R. Razouk and B. Deal, "Dependence of Interface State Density on Silicon Thermal Oxidation Process Variables," Journal of the Eletrochemıcal Socıety, vol. 126, September 1979.

[40] Y. Fujikawa, Y. Yamada-Takamura, and G. Yoshikawa, "Silicon on insulator for symmetry-converted growth," Applıed Physıcs Letters, vol. 90, no. 243107, 2007.

[41] H. Shichijo, R. Matyi, and A. Taddiken, "Monolithic Process for co-inegration of GaAs MESFET and silicon CMOS Devices and Circuits," IEEE Transactıons on Electron Devices, vol. 37, March 1990.

[42] K. Matsunaga, M. Tanomura, and T. Nakayama, "Analogue Dynamic Supply Voltage L-band GaN High Power Amplifier with Improvement of Efficiency and Linearity," Mıcrowave Symposıum 2007. IEEE/MTT-S Internatıonal, pp. 11071110, June 2007.

[43] J. Chung, J.-K. Lee, E. Piner, and T. Palacios, "Seamless On-Wafer Integration of $\mathrm{Si}(100)$ MOSFETs and GaN HEMTs," IEEE Electron Device Letters, vol. 30, October 2009.

[44] D. Pastor and R. Cusco, "The effect of substrate on high-temperature annealing of GaN epilayers: Si versus Sapphire," Journal of Applzed Physics, vol. 100, no. 043508, 2006. 
[45] D. Koleske and A. Wickenden, "GaN decomposition in $\mathrm{H} 2$ and N2 at MOCVD temperatures and pressures," Journal of Crystal Growth, vol. 223, pp. 466-483, 2001.

[46] A. Pisch and R. Schmid-Fetzer, "In situ decomposition study of GaN thin films," Journal of Crystal Growth, vol. 197, pp. 329-332, 1998.

[47] Y. Song, X. Wu, and W. Wang, "Thermal Stability and electronic specific heat of GaN," Journal of Alloys and Compounds, vol. 370, pp. 65-68, 2004.

[48] A. Stonert and K. Pagowska, "Channeling study of thermal decomposition of III-N compund semiconductors," Nuclear Instruments and Methods in Physics Research B, vol. 266, pp. 1224-1228, 2008.

[49] Y. Irokawa, O. Ishiguro, and T. Kachi, "Implantation temperature dependence of Si activation in AlGaN," Applied Physıcs Letters, vol. 88, no. 182106, 2006.

[50] K. Lorenz and U. Wahl, "High temperature annealing of rare earch implanted GaN films: Structural and optical properties," Optıcal Materıals, vol. 28, pp. $750-758,2006$.

[51] P. Chyurlia, "Thermal Analysis of AlGaN/GaN HEMT monolithic integration with CMOS on Silicon < $111>$ substrates," Master's thesis, Carleton University, 2006.

[52] J.-M. Bethoux and P. Vennegues, "Growth of high quality crack-free AlGaN films on GaN templates using plastic relaxation through buried cracks," Journal of Appl. Phys., vol. 94, no. 6499, 2003.

[53] D. Cheng and M. Gonsalvez, "Improvement in smoothness of anisotropically etched silicon surfaces: Effects of surfactant and TMAH concentrations," Sensors and Actuators A, vol. 125, pp. 415-421, 2006.

[54] D. Resnick and D. Vrtacnick, "Effective roughness reduction of (100) and (311) planes in anisotripic etching of (100) silicon in 5 percent TMAH," J. Micromech. Microeng., vol. 13, pp. 26-34, 2003.

[55] R. Houbertz and U. Memmert, "On the potential-dependant etching of $\mathrm{Si}(111)$ in aqueous $\mathrm{NF}_{4} \mathrm{~F}$ solution," Surface Scıence, vol. 396, pp. 198-211, 1998.

[56] H. Fukidome and M. Matsumura, "Electrochemical study of atomically flattening process of silicon surface in 40 percents $\mathrm{NF}_{4} \mathrm{~F}$ solution," Applzed Surface Scıence, vol. 130, pp. 146-150, 1998. 
[57] H. Lewerz and H. Jungblut, "Surface Analysis of the eletropolishing layer on $\mathrm{Si}(111)$ in ammonium flouride solution," Electrochımıca Acta, vol. 45, pp. 4615$4627,2000$.

[58] J. Bardwell and S. Haffouz, "AlGaN/GaN HFETs fabricated from maskless selectively grown mesas on $\mathrm{Si}(111)$ substrates," Electronics Letters, vol. 43, October 2007.

[59] P. Chyurlia, F. Semond, T. Lester, J. Bardwell, and N. Tarr, "Windowed growth of AlGaN/GaN Heterostructures on Silicon $<111>$ substrates for Future MOS Integration," Physıca status solıdı a, vol. 206, no. 2, pp. 371-374, 2009.

[60] E. Kasper and H. Herzog, "Monolithic integration using differntial Si-MBE," Journal of Crystal Growth, vol. 81, pp. 458-462, 1987.

[61] F. Semond and Y. Cordier, "Molecular Beam Epitaxy of Group-III Nitrides on Silicon Substrates: Growth, Properties and Device Applications," Physıca Status Solıd, , vol. 188, pp. 501-510, 2001.

[62] G. Marlow and M. Das, "The Effects of Contact size and non-zero Metal Resistance on the determination of Specific Contact Resistance," Solvd-State Electronıcs, vol. 25, no. 2, pp. 91-94, 1982.

[63] E. Frayssinet, W. Knap, and S. Krukowski, "Evidence of free carrier concentration gradient along the c-axis for undoped GaN single crystals," Journal of Crystal Growth, vol. 230, pp. 442-447, 2001.

[64] W. Kim, A. Botchkarev, and A. Salvador, "On the incorporation of $\mathrm{Mg}$ and the role of oxygen, silicon and hydrogen in $\mathrm{GaN}$ prepared by reactive molecular beam epitaxy," Journal of Applied Physics, vol. 82, July 1997.

[65] J. Oila and V. Ranki, "Influence of dopants and substrate material on the formation of Ga Vacanacies in epitaxial GaN layers," Physıcal Revıew B, vol. 63, no. $045205,2001$.

[66] C. V. de Walle, "Effects of impurities on the lattice parameters of GaN," Physıcal Revrew B, vol. 68, no. 165209, 2003.

[67] "Siltronix wafer specifications." http://www.siltronıx.com/wafer.php.

[68] H. Tang, S. Rolfe, J. Baribeau, J. Bardwell, P. Chyurlia, and N. Tarr, "Growth of GaN/AlGaN HEMT on Si (110) for integration with silicon electronics," MBE, 2010 international conference, submitted April 2010. 
[69] Unknown, "Taurus tsuprem-4," 2011.

[70] C. Sah, "Electronic Processes and Excess Currents in Gold-Doped Narrow Silicon Junctions," Physics Review, vol. 123, pp. 1594-1612, 1961.

[71] "Sonnet high frequency electromagnetic software." http://www. sonnetsoftware.com/.

[72] T. Blank and Y. Gol'dberg, "Mechanisms of Current Flow in MetalSemiconductor Ohmic Contacts," Semıconductors, vol. 41, no. 11, pp. 1263-1292, 2007.

[73] F. Locolano, F. Roccaforte, and V. Ranieri, "Temperature dependence on the specific resistance in $\mathrm{Ti} / \mathrm{Al} / \mathrm{Ni} / \mathrm{Au}$ contacts on n-type GaN," Journal of Applied Physıcs, vol. 100, no. 123706, 2004.

[74] M. Miller and S. Mohney, "V/Al/V/Ag Ohmic contacts to n-AlGaN/GaN heterostructures with a thin GaN cap," Applzed Physics Letters, vol. 91, no. 012103, 2007.

[75] A. Grove, Physıcs and Technology of Semıconductor Devıces. Wiley, 1967.

[76] A. Joshi and D.-L. Kwong, "Study of the Growth Temperature Dependence of Performance and Reliability of Thin MOS Gate Oxides," IEEE trans. on Electronıc Devices, vol. 39, September 1992.

[77] S. Takagi, A. Toriumi, and H. Tango, "On the Universality of Inversion Layer Mobility in Si MOSFETs: Part II - Effects of Surface Orientation," IEEE trans. on Electron Devıces, vol. 41, December 1994.

[78] H. Hasegawa, T. Inagaki, S. Ootomo, and T. Hashizume, "Mechanisms of current collapse and gate leakage currents in $\mathrm{AlGaN} / \mathrm{GaN}$ heterostructure field effect transistors," Journal of Vacuum Scrence and Technology, vol. 21, no. 4, pp. 1844$1855,2003$.

[79] K. Dora, A. Chakraborty, and U. Mishra, "High Breakdown Voltage Achieved on AlGaN/GaN HEMTs with Integrated Slant Field Plates," IEEE Electron Device Letters, vol. 27, September 2006.

[80] M. Stutzmann, G. Steinhoff, M. Eickhoff, O. Ambacher, C. E. Nebel, J. Schalwig, R. Neuberger, and G. Mller, "Gan-based heterostructures for sensor applications," Diamond and Related Materıals, vol. 11, no. 3-6, pp. 886 - 891, 2002. 
Appendix A

Tsuprem Input File for Original nMOS

Design 


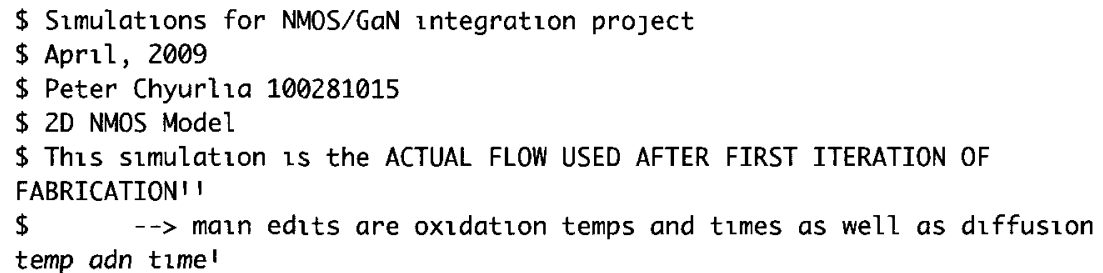

$\$$ Full non-equilibrium point defect model $\$$ (uncludes transient enhanced diffusion) method pd.full

$\$$ Graphics written as postscript file option device="c/postscript" plot.out="2D_NMOS ps"

$\$-$

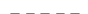

\$ MESH DEFINITION

Sonly specify half of the design and then reflect in the Y-axis $\$$ Speclfy the Mesh

MESH GRID.FAC $=1.5$

MESH DY.SURF $=0.002 \quad$ LY.SURF $=002 \quad$ LY.ACTIV $=2.0$

$\$-$

\section{- - -}

\$INITIALIZE WAFER

\$Set the well background dopung

$\$$ This is the doping of the p-type wafer

$\$<111>$ added to say that we are using 111 type silicon

$\$$ width now changed to 13.5 since we need contacts 5 um from gate and 5 um wide contacts

unttualıze Boron=1.3E12<111> WIDTH=13.5 DX=0.05

\$ Plot inztial Mesh

SELECT TITLE="NMOS MESH"

PLOT.2D SCALE GRID Y.MAX $=3$ C.GRID $=2$

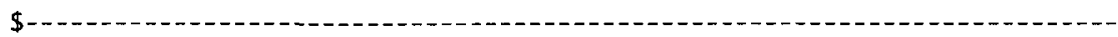


\$WELL IMPLANT

\$need a cap oxide for implant 200 Angstroms

Deposit oxide thick $=0.02$

$\$$ umplant Boron to form the well

$\$$ goal is $1 \mathrm{e} 16$ surface conc.

$\$$ use singly/doubly zonzzed boron and a high energy such that we don't need an anneal

\$ go back to tr.boron pearson model, the chboron is giving weird behaviour $\$$ Can't, CHBORON is only one that goes past $100 \mathrm{keV}$, use it for this only

$\$$ can't used triply ionized, BUT, 300 is still ok for doubly zonzzed!

implant boron dose $=6 \mathrm{E} 11$ energy $=300 \mathrm{tilt}=7$ rotation $=30 \mathrm{impl}, \mathrm{tab}=$ chboron

$\$$ Plot the as-implanted boron distribution

select $\quad z=\log 10$ (boron) TITLE="Retrograde P-WELL"

plot.1d top=17 bottom=14 right=1.3 color $=2$ line.typ=1 $X$. VALUE $=10$

\$second implant for retrograde well

\$Still using chboron due to high energy implant

$\$$ this can be singly ionized since we can go up to $180 \mathrm{keV}$

Implant boron dose $=3 \mathrm{E} 11$ energy $=120 \mathrm{tl} \mathrm{l} t=7$ rotation $=30 \mathrm{impl} . \mathrm{tab}=$ chboron

$\$$ plot the second implant

select $\quad z=\log 10$ (boron)

plot 1d $\wedge a \times \wedge c l$ color $=3$ line. $t y p=2 \times$. VALUE $=10$

\$third implant for retrograde well



$\$$ Plot the as-implanted boron distribution for retrograde well

select $z=\log 10$ (boron)

plot.1d ^ax ^cl color $=4$ line.typ $=3 \quad x$. VALUE $=10$

etch oxide

$\$-----$

\$ P-Gaurd PE - this gives Vth of about $19 \mathrm{~V}$, wh thout it we get $4.9 \mathrm{~V}$, which is ok 


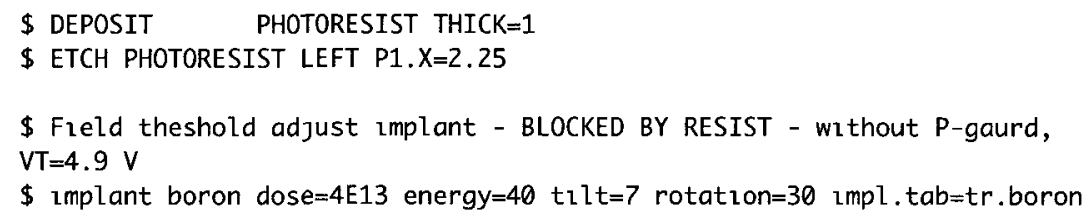

$\$-$

\$Field oxidation

\$pad oxide

deposit oxide thick $=0.02$

Sdeposit pad nitride

deposit nutride thick $=0.008$

\$etch nutride in outer edge to form FOX

etch Nitride LEFT P1.X=2.25

\$Field oxidation - results in 0.5 um thick field oxide

$\$$ done at 950 actually, for a long time to give around $0.5 \mathrm{mlcrons}$

$\$$ performed for 1 hour and 53 minutes $->$ GaN exploded here

DIFFUSION TEMP $=950$ TIME $=113$ WETO2

\section{\$ REMOVE PAD NITRIDE}

ETCH NITRIDE ALL

$\$-$

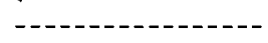

\$Finish Plotting P-Well Concentrations

$\$$ Check that all the boron is electrically active

select $z=\log 10$ (active(boron))

plot.1d ^ax $\wedge$ cl color $=6$ lne. $t y p=5$ X. VALUE $=10$

\$Add a legend to the plot

LABEL CM X $X=11 \quad Y=23$

Line.typ=1 LEFT

LABEL CM X $=11 \quad \mathrm{Y}=22.5 \quad$ COLOR=3 LABEL="Second Implant - $120 \mathrm{KeV} "$

Line. typ $=2$ LEFT

LABEL CM X=11 Y=22 COLOR=4 LABEL="Thurd Implant - $30 \mathrm{KeV"}$

Line. typ $=3$ LEFT

LABEL CM X=11 $Y=21.5 \quad$ COLOR=6 LABEL="Active Boron" Line. typ=5

LEFT 
\$Print junction depth information

select $\quad z=$ doping

print.1d layers

Setch away the screen oxide $200 \mathrm{~A}$

etch oxide thick $=0.03$

$\$$

-

\$GATE FORMATION

\$gate oxidation

$\$$ done at 950 , not 1000 , we got $311 \mathrm{~A}$, not 250 so see what we get here

DIFFUSION TEMP $=950$ TIME $=24$ DRYO2

\$oxide anneal (10 mins only)

Diffusion TEMP $=950$ Time $=10$

\$deposit Poly

DEPOSIT POLYSILICON THICKNESS $=0.4$ DIVISIONS $=4$

\$Poly and oxtde etch between 0.5 and $12.25 \mathrm{mLcrons}$

$\$$ this way, reflecting left will give a parasitic transistor and reflecting right will

$\$$ give the actual device

\$ETCH POLY LEFT P1.X=12.25

ETCH POLY START $X=0.75 Y=-2$

ETCH CONTINUE $X=0.75 Y=2$

ETCH CONTINUE $X=12.25 \quad Y=2$

ETCh DONE $X=12.25 \quad Y=-2$

Setch away oxide in S/D windows

ETCH OXIDE TRAP thick $=0.03$

$\$$

\$source and drain formation

\$phosphorus diffusion

$\$$ done at 900 for $20 \mathrm{mun}-\rightarrow$ got $18 \mathrm{ohms} / \mathrm{sq}$ in test wafer

$\$$ I. CONC $=1 \mathrm{e} 21$ is only giving $45 \mathrm{ohm} / \mathrm{sq}$, try upping this

$\$ I . C O N C=1 e 22$ is giving $33 \mathrm{ohm} / \mathrm{sq}$ try upping again

diffusion time $=20$ temp $=900$ Impurity=Phosphorus I. CONC $=1 \mathrm{e} 23$

$\$$ Drive-ın 
diffusion temp $=900$ tıme $=5$
\$Back END
\$BPS deposition - very thick for this process
DEPOSIT OXIDE THICK $=0.6$
\$Thermal steps associated with BPSG
DIFFUSION time=20 temp=900
\$LOng Time hydrogen annealing step - not done
\$DIFFUSION time=240 temp=450
\$-

$\$ G a N$ Processing Steps at this point

\$Ohmrc metal Anneal --> leave out, does nothing

\$Diffusion temp $=750$ trme $=\emptyset .5$

$\$-$

Sopen up wzndows for fall

ETCH OXIDE START $X=2.25 \quad Y=-2$

ETCH CONTINUE $X=2.25 Y=2$

ETCH CONTINUE $X=7.25 Y=2$

ETCh DONE $X=7.25 \quad Y=-2$

\$Metall ization

Deposit Alumınum THICK=1 SPACES=3

DEPOSIT PHOTORESIST THICK $=1$

ETCH PHOTORESIST RIGHT P1. $X=7.5$

ETCH PHOTORESIST LEFT P1. $X=1.5$

ETCH ALUMINUM TRAP ANGLE $=85$ THICK $=1.5$

ETCH PHOTORESIST ALL

$\$-$

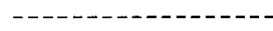

SREFLECT FILE

SAVEFILE OUT.FILE=NMOS_HALF.STRUCT

STRUCTURE REFLECT RIGHT

SAVEFILE OUT.FILE=2D_NMOS.MEDICI MEDICI

$\$-$

$\$ P l o t$ structure 


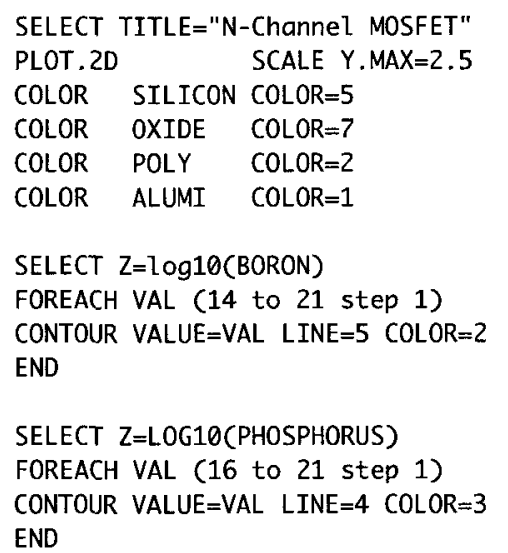

$\$$

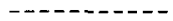

\$Find Vt

\$output threshold voltage

SWF is work function in ev 4.35 corresponds to doping > 1 E20

ELECTRIC $X=13.5$ THRESHOLD NMOS V="0 2 .05" GATE. WF $=4.35$ GATE. ELE

\$plots sheet conductance vs voltage

PLOT.1D ELECTRIC

$\$-$

\$Plot Sheet resistance

electric $x=10$ resistan

\$-1

$\$$ Find parasitic Vt by reflecting other way

LOADFILE IN.FILE=NMOS_HALF.STRUCT

STRUCTURE REFLECT LEFT

\$Plot structure 


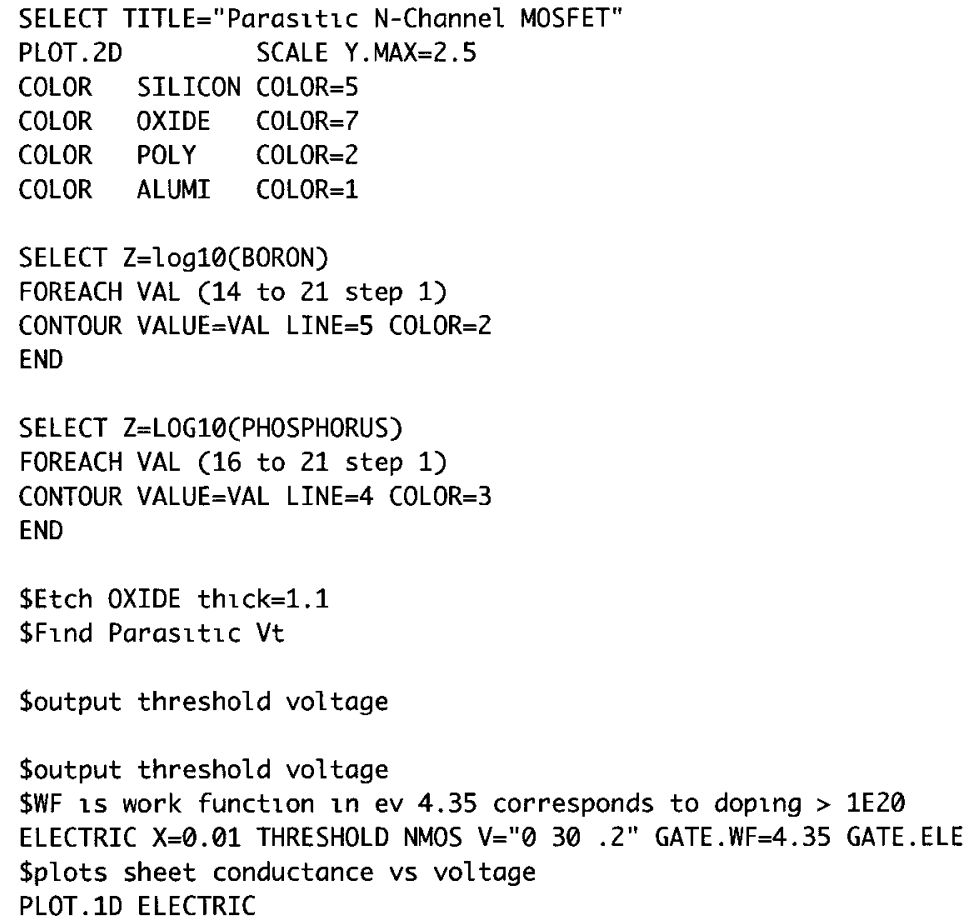




\section{Appendix B}

\section{Medici Input file used to calculate MOS device properties}

Files in following order:

Setup file

Subthreshold Simulation

Threshold Voltage Determination

Saturation Curves

Punchthrough

Hot Carrier effects 


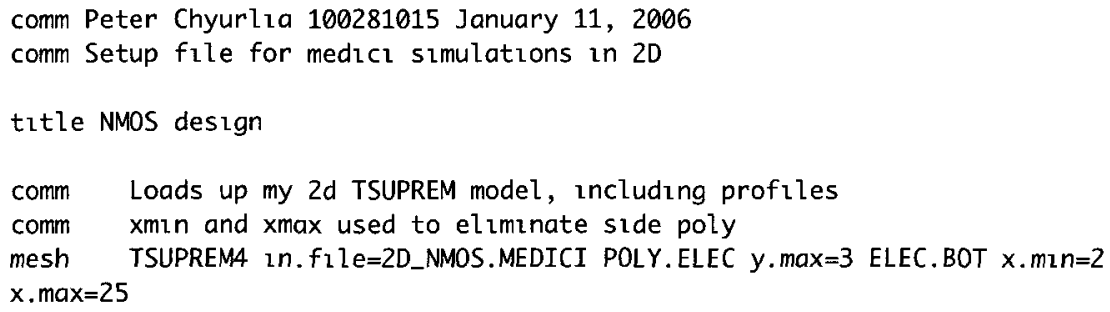

RENAME ELECTR OLDNAME=1 NEWNAME=source RENAME ELECTR OLDNAME $=2$ NEWNAME $=$ drain comm ELEC.BOT renames the bottom to an electrode called substratel RENAME ELECTR OLDNAME=Substrate NEWNAME=sub

contact number $=$ gate $n$. poly WORKFUNC $=4.35$

plot.2d grid boundary fill scale

comm regrid on doping, ignore region 1

comm use quad-tree mesh now

regrid QT.REGRI doping ignore $=(1) \log$ ratıo=2 smooth $=1$

plot.2d grid boundary fill scale

contact name=gate $n$. poly

models conmob fldmob srfmob2

symb carriers $=0$

method $1 \mathrm{ccg}$ damped

solve

comment regrid on potential twice

regrid QT.REGRI potent $\mathbf{g}$ gnore $=(1)$ ratıo=0.2 $\max =1$ smooth $=1$

plot.2d grid boundary fill scale 
regrid QT.REGRI potent ignore=(1) ratio=0.1 $\max =1$ smooth $=1$ out. $f \mathrm{l}$ le $=$ nmos_mesh

plot.2d grid boundary fill scale

comm 2D concentration

plot.2d boundary fill scale

contour doping LOG MIN=-20 MAX=-14 DEL=0.5 COLOR=2 LINE=2 contour doping $L O G$ MIN=16 MAX $=21$ DEL=0.5 COLOR=1 LINE=2

comm Plot1D concentration -- at $y=0$ it should be just under 10E15

plot.1d DOPING Y.Start $=-0.0253$ Y.END $=0.2 X$. start $=5 X$. end $=5$ POINTS Y.LOG

$+\quad$ color $=2$

comm + device $=" c /$ postscrupt" plot. out=grid.ps

comment solve for zero bias and save

symb carrers $=0$

solve out.file=nmos_solution 


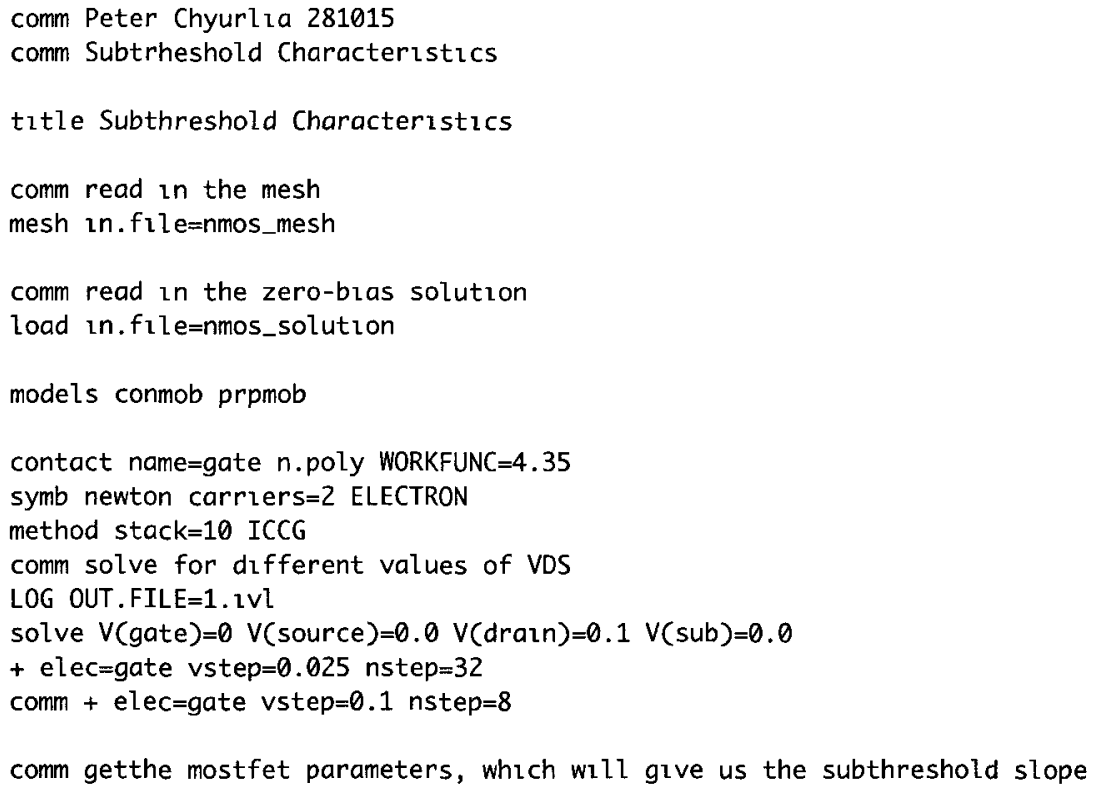

mesh $2 n . f 2 l e=n m o s \_m e s h$ 
load in.file=nmos_solution

contact name $=$ gate $n$.poly WORKFUNC $=4.35$

symb newton carriers $=2$ ELECTRON

method stack $=10$

LOG OUT.FILE $=3.1 \mathrm{vl}$

solve $V($ gate $)=0$ V(source $)=0.0 V($ drain $)=5 V($ sub $)=0.0$

+ elec $=$ gate vstep $=0.025$ nstep $=32$

comm getthe mostfet parameters, which will give us the subthreshold slope

EXTRACT MOS.PARA

mesh in.file=nmos_mesh

load in.file=nmos_solution

contact name $=$ gate $n$. poly WORKFUNC $=4.35$

symb newton carriers=2 ELECTRON

method stack $=10$

LOG OUT. FILE $=4$. IVI

solve $V($ gate $)=0 \mathrm{~V}($ source $)=0.0 \mathrm{~V}($ dratn $)=10 \mathrm{~V}($ sub $)=0.0$

+ elec $=$ gate vstep $=0.025$ nstep $=32$

comm getthe mostfet parameters, which will give us the subthreshold slope

EXTRACT MOS.PARA

comm Plot everything

plot.1d $x \cdot a x=V($ gate $)$ y.ax=l(drain) y. $\log$ Line. $t y p=1$ in. fl le $=1.2 v l$

$+\quad$ title="Subthreshold Characterıstıcs"

$+\quad$ device="cl/postscrupt" plot.out=subthreshold_curves.ps

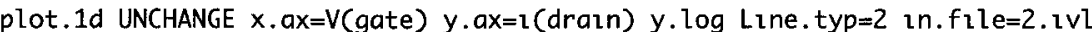

plot. 1d UNCHANGE $x . a x=V($ gate $) y . a x=1(d r a i n) y . \log$ Line.typ=3 in.f $q l e=3 . \imath v l$

plot.1d UNCHANGE $x . a x=V($ gate $) y . a x=\imath(d r a i n)$ y. $\log$ Line. $t y p=4$ in. $f \imath l e=4 . \imath v l$

comm Label all the plots

LABEL LABEL="Vds $=0.1 \mathrm{~V} "$ Line. typ=1 C.Size $=0.5$ Start.LE LX.FINIS=10 CM

$+\quad X=13 \quad Y=6$

LABEL LABEL="Vds $=1 \mathrm{~V} "$ Line. $t y p=2 \quad$ C.Size $=0.5$ START.LE LX. FINIS $=10 \mathrm{CM}$

LABEL LABEL="Vds $=5 \mathrm{V"}$ Line. $t y p=3 \mathrm{C}$. S $z=0.5$ START.LE LX.FINIS $=10 \mathrm{CM}$

LABEL LABEL="Vds $=10 \mathrm{~V} "$ Line. typ $=4 \mathrm{C}$. Size $=0.5$ START.LE LX.FINIS $=10 \mathrm{CM}$ 


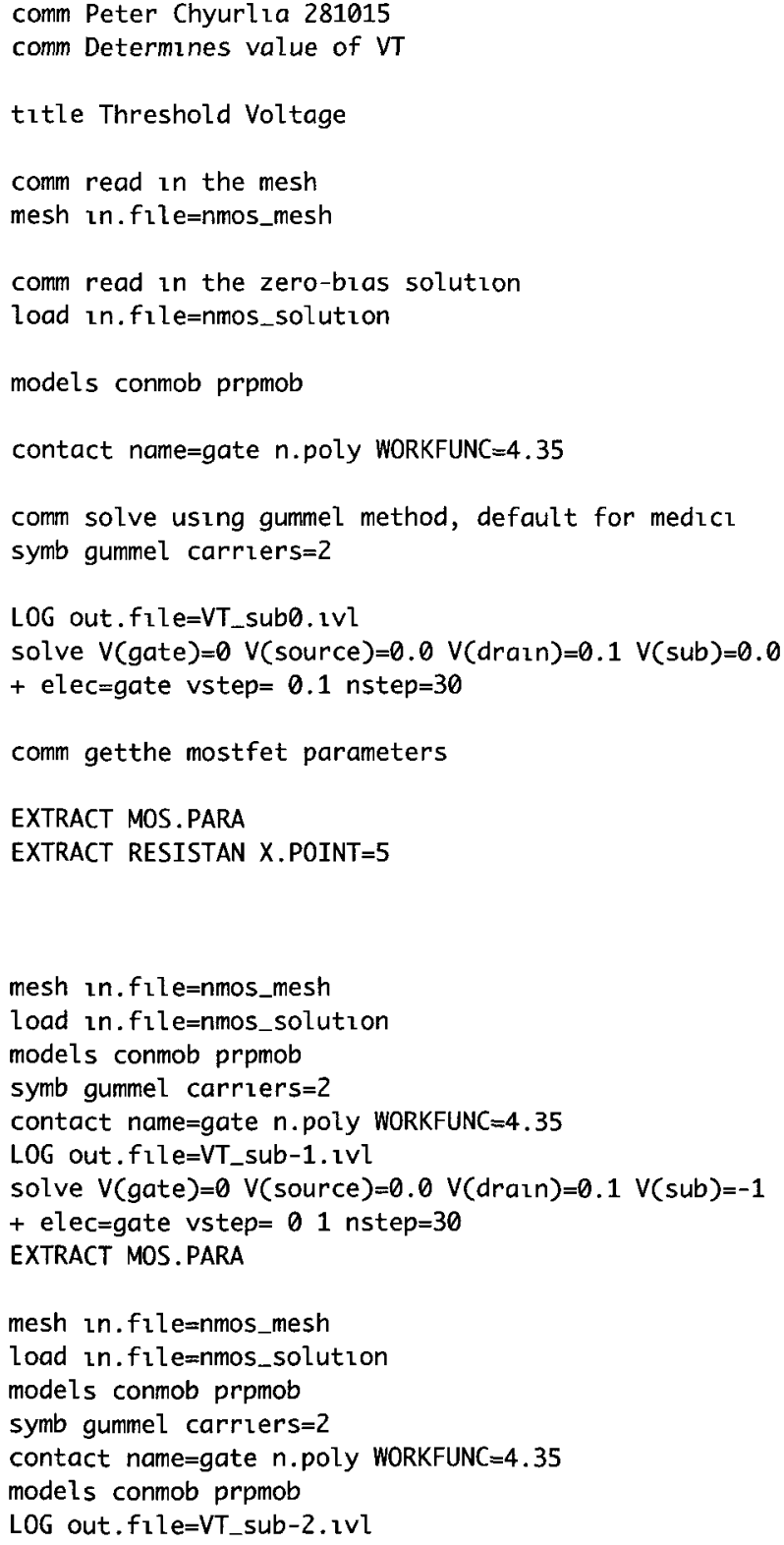


solve $V($ gate $)=0 V($ source $)=0.0 V($ drain $)=0.1 V($ sub $)=-2$

+ elec $=$ gate vstep $=0.1$ nstep $=30$

EXTRACT MOS.PARA

comm plot the output

Plot.1D Y.AXIS=I(drain) X.Axis=V(gate) POINTS Color $=2$ IN.FILE=VT_sub0.ivl

$+\quad$ TITLE="ID vs VGS Curve" device="c/postscript"

plot .out=IDvVG_threshold.ps

Plot.10 Y.AXIS=I(drain) X.AXis=V(gate) POINTS Color=2

IN.FILE=VT_sub-1.ivl UNCHANGE

Plot.1D Y.AXIS=I(drain) X.AXis=V(gate) POINTS Color=2

IN.FILE=VT_sub-2.ivl UNCHANGE

\section{comm}

comm put plot into IC-CAP format

LOG ICCAP IN.FILE=VT_sub 0 . ivl OUT.FILE=vt_curve_VD01_VBO. set

+ DRAIN=drain GATE=Gate SOURCE=source SUBSTRAT=sub

+ OUT1=I(drain) INP1=V(gate) INP2=V(drain)

$+\mathrm{INP} 3=\mathrm{V}($ sub $)$ INP4 $=\mathrm{V}$ (source)

LOG ICCAP IN.FILE=VT_sub-1. ivl OUT.FILE=Vt_curve_VD01_VB-1. set

+ DRAIN=drain GATE $=$ Gate SOURCE $=$ source SUBSTRAT $=$ sub

+ OUT1=I(drain) INP1=V(gate) INP2=V(drain)

+ INP3 $=V($ sub $)$ INP4 $=V($ source $)$

LOG ICCAP IN.FILE=VT_sub-2.ivl OUT.FILE=vt_curve_VD01_VB-2.set

+ DRAIN=drain GATE=Gate SOURCE=source SUBSTRAT=sub

+ OUT1=I(drain) INP1=V(gate) INP2=V(drain)

+ INP3 $=V($ sub) INP4 $=$ V(source)

end 


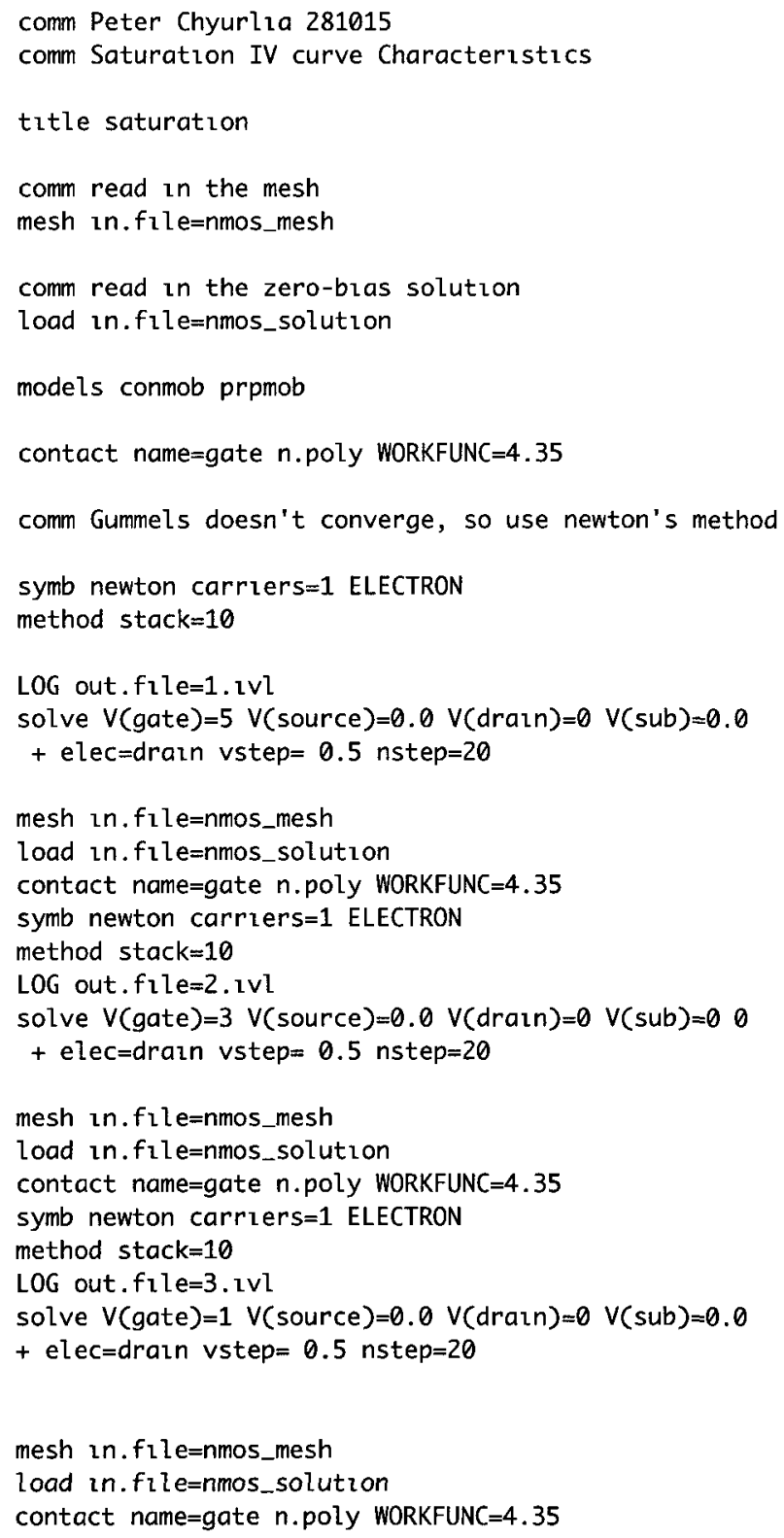







comm Peter Chyurlia 281015

comm Punthrough Simulation

title Punch-through Simulation

comm read in the mesh

mesh in.file=nmos_mesh

comm read in the zero-bias solution

load in.file=nmos_solution

models conmob prpmob

contact name $=$ gate $n$.poly WORKFUNC $=4.35$

comm solve using newton's method

symb newton carriers $=1$

method stack $=10$

comm no gate or source voltage, adjust VDS

comm want less than $10 \mathrm{nA} / \mathrm{um}$

solve $V($ gate $)=0 V($ source $)=0.0 V($ drain $)=59 V($ sub $)=0.0$

+ elec $=$ draln vstep $=1$ nstep $=10$

comm getthe mostfet parameters

EXTRACT MOS. PARA

comm plot the output

Plot.10 Y.AXIS=I(drain) X.Axıs=V(drain) POINTS Color $=2$

$+\quad$ TITLE="ID vs VDS Curve" X.MIN=60

$+\quad$ device $=" c l /$ postscript" plot.out=punchthrough.ps

plot.2d boundary fill scale REGION JUNCTION title="Potentlal Contours, $v g=0, v d=66^{\prime \prime}$

contour potentıal

plot.2d boundary fill scale REGION JUNCTION title="Electron concentration, $v g=0, v d=66^{\prime \prime}$

contour electron logarith min.valu=15 max.valu=20 del.valu=1 


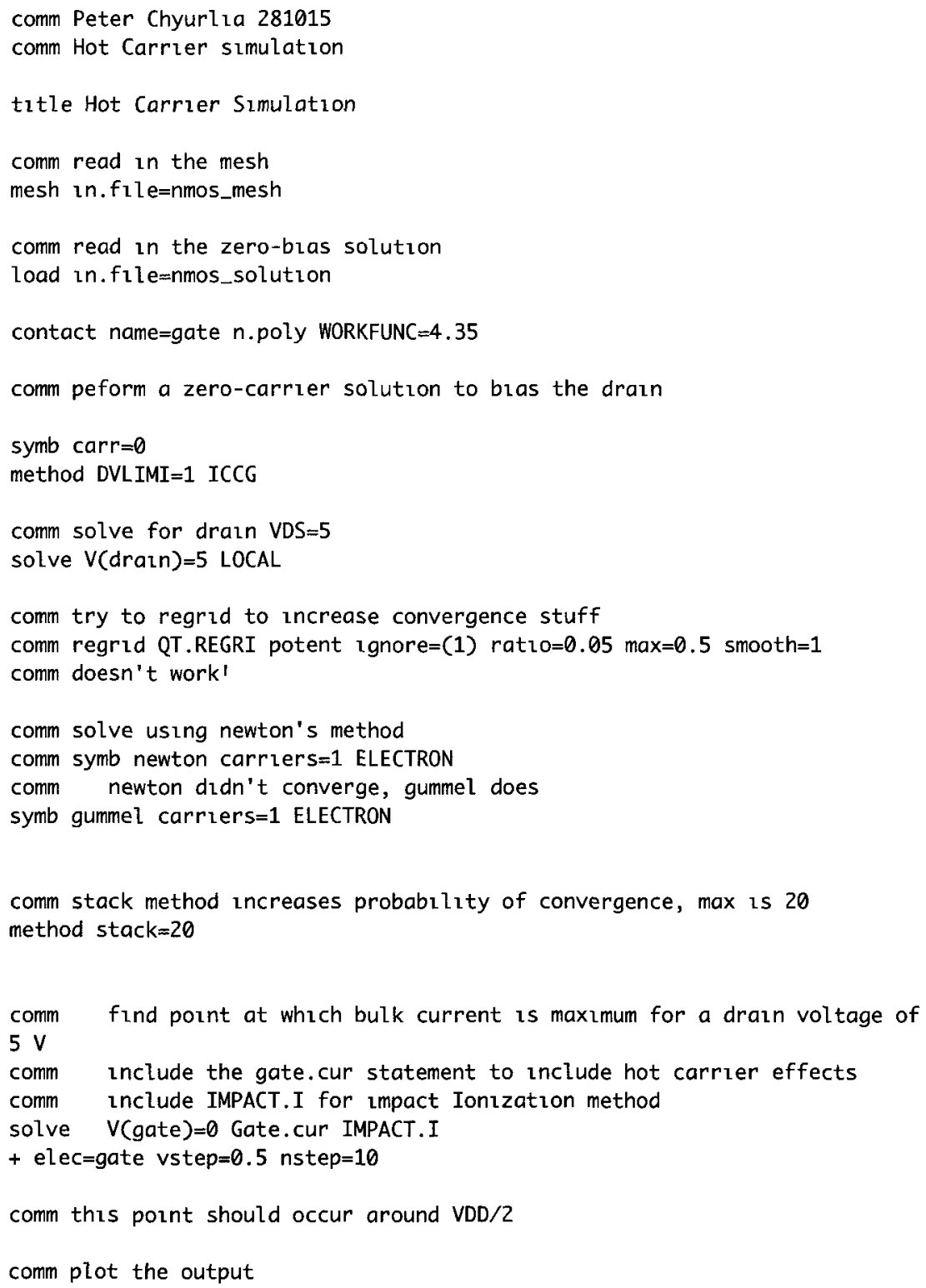




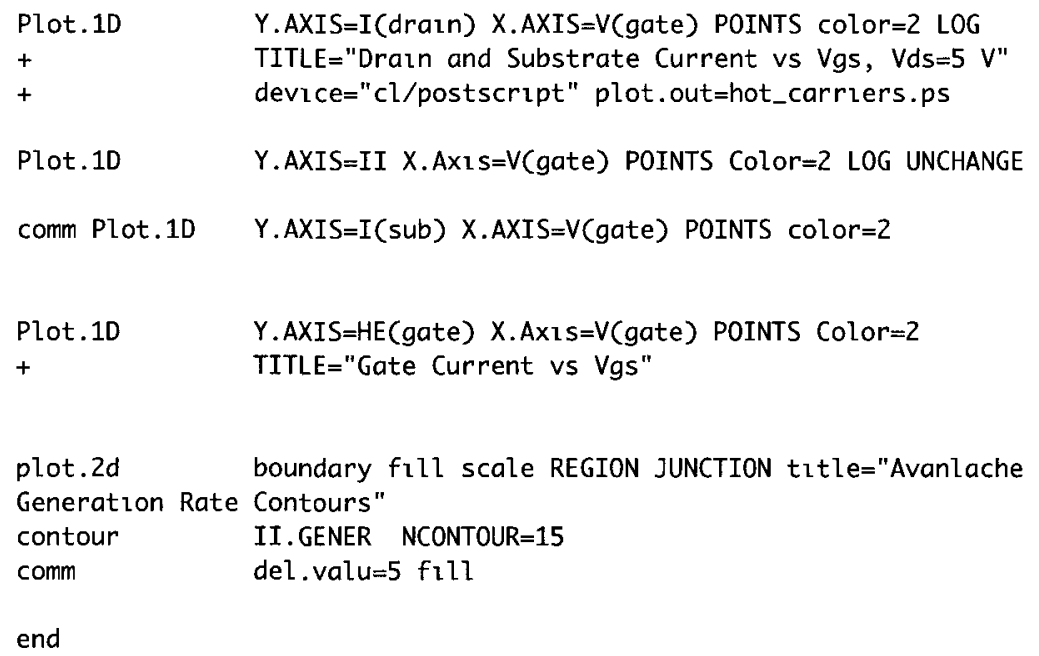


Appendix C

Process Sheet for Fabrication Process

C.1 Enclosed Process Flow 
484L - no NRC details

\begin{tabular}{|lll}
\hline LOG\# & $484 \mathrm{~L}$ & PROJECT CODE \\
MATERIAL & GaN on $S 1<110>$ and $<111>$ & PHONE \\
SAMPLE ID & A-E plus 2 test quarters (110) and (111) & DATE \\
DESTINATION & Jennifer/Peter & \\
\hline \multicolumn{3}{|c}{ FInal ENLOSED Process, includes changes made from } \\
& 464 L and $458 \mathrm{~L} 900 \mathrm{C}$ ready
\end{tabular}

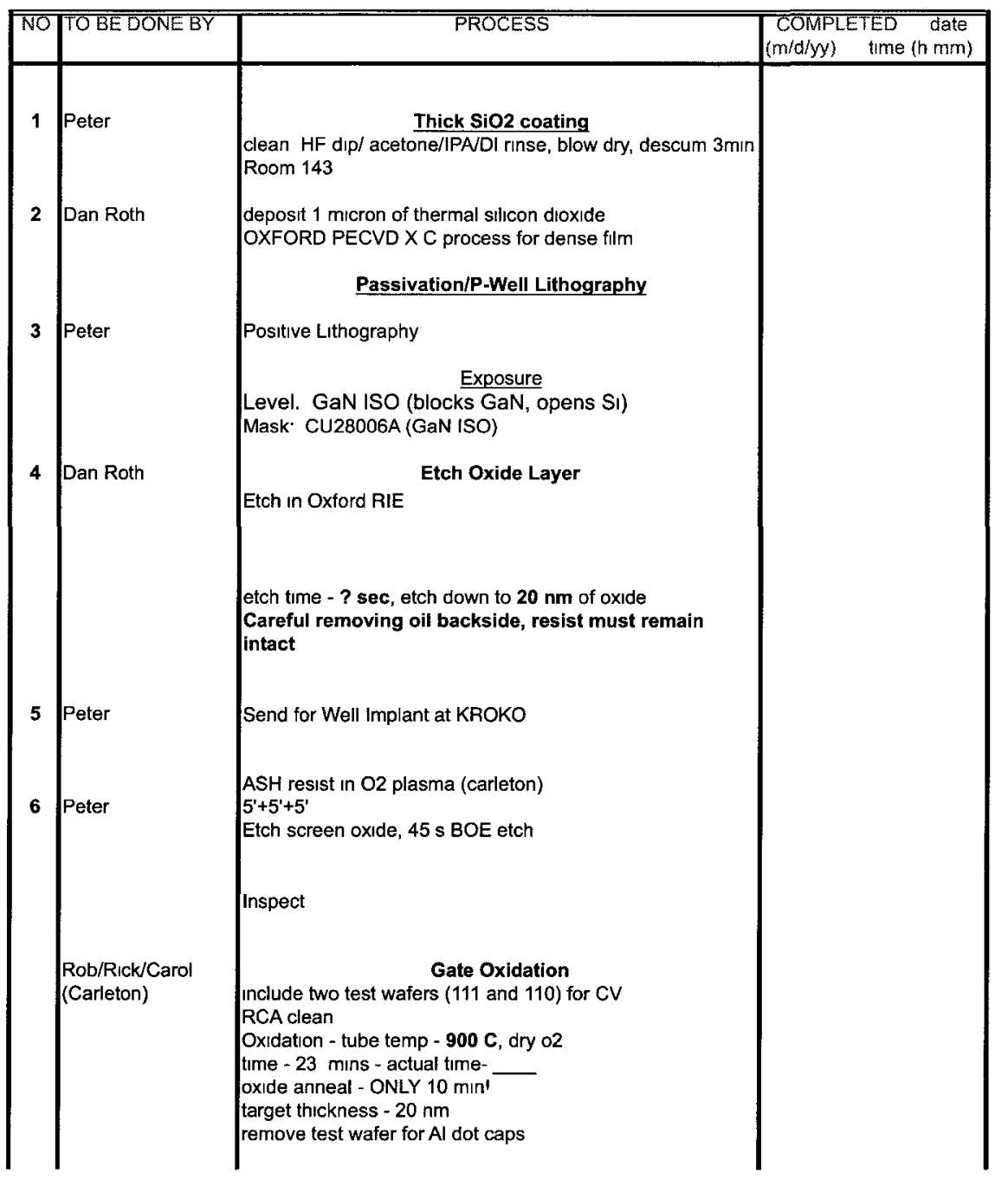


484L - no NRC details

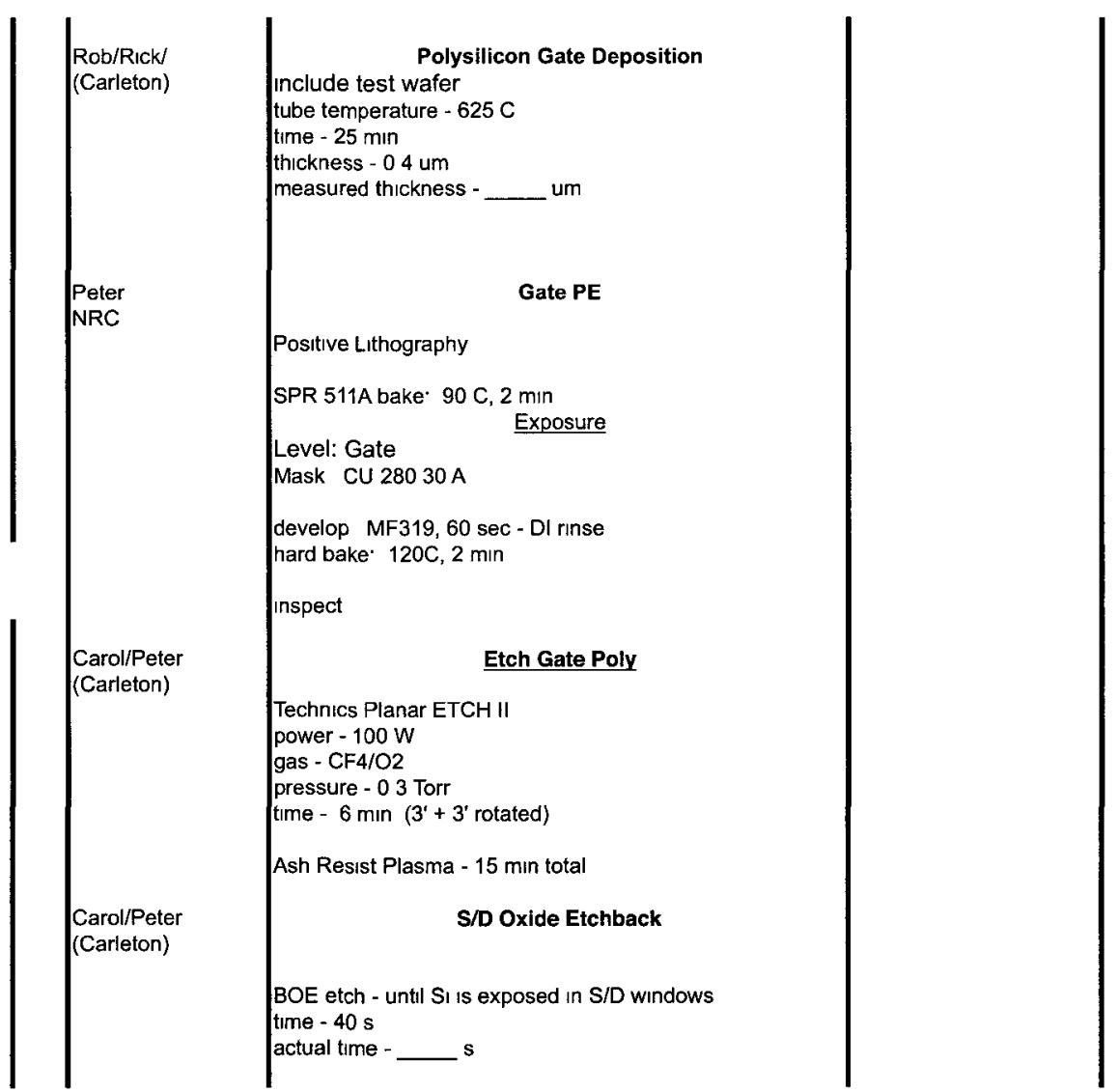


484L - no NRC details

484L - no NRC detalls

\begin{tabular}{|c|c|}
\hline I & $10 \mathrm{~min}$ cool \\
\hline \multirow{3}{*}{$\begin{array}{l}\text { Carol/Peter } \\
\text { (Carleton) }\end{array}$} & GaN Recovery \\
\hline & GaN Recovery PE \\
\hline & $\begin{array}{l}\text { Positive Lithography } \\
\text { Level Silıcon Isolatıon } \frac{\text { Exposure }}{(\text { blocks SI, open GaN) }} \\
\text { Mask Cu28005A (Silicon Iso) }\end{array}$ \\
\hline \multirow{2}{*}{$\begin{array}{l}\text { Dan Roth } \\
\text { (NRC) }\end{array}$} & BPSG Etch/Passivation Etch \\
\hline & $\begin{array}{l}\text { Etch in Oxford RIE } \\
\text { press - } 10 \text { mTorr, temp } 35 \mathrm{C} \\
\mathrm{O} 2-55, \mathrm{Ar}-50, \mathrm{C} 4 \mathrm{~F} 8-25(\mathrm{sccm}) \\
\text { ICP }-1500 \text { watts, if }-80 \text { watts } \\
\text { etch tIme - sec, to remove } 04 \text { um BPSG + } \sim 05 \text { um } \\
\text { thermal PECVD oxide } \\
\text { use oil - LEAVE at least } 1000 \text { A OXIDE ON GAN } \\
\text { SURFACE - Etching to GaN ruins samples! }\end{array}$ \\
\hline Peter & $\begin{array}{l}\text { BOE w/surfacant etch to reveal GaN } \\
\text { etch rate should be around } 800 \mathrm{~A} / \mathrm{min} \\
\text { t/me= }\end{array}$ \\
\hline PETER & $\begin{array}{l}\text { Measure 2DEG in HALL ROOM } \\
\text { Start of GaN Processing }\end{array}$ \\
\hline \multirow[t]{2}{*}{$\begin{array}{l}\text { Peter } \\
\text { (carleton) }\end{array}$} & $\begin{array}{l}\text { MESA lithography } \\
\text { Ash Resist Plasma - } 15 \text { min total }\end{array}$ \\
\hline & |Positive Lithography \\
\hline
\end{tabular}


484L - no NRC detals

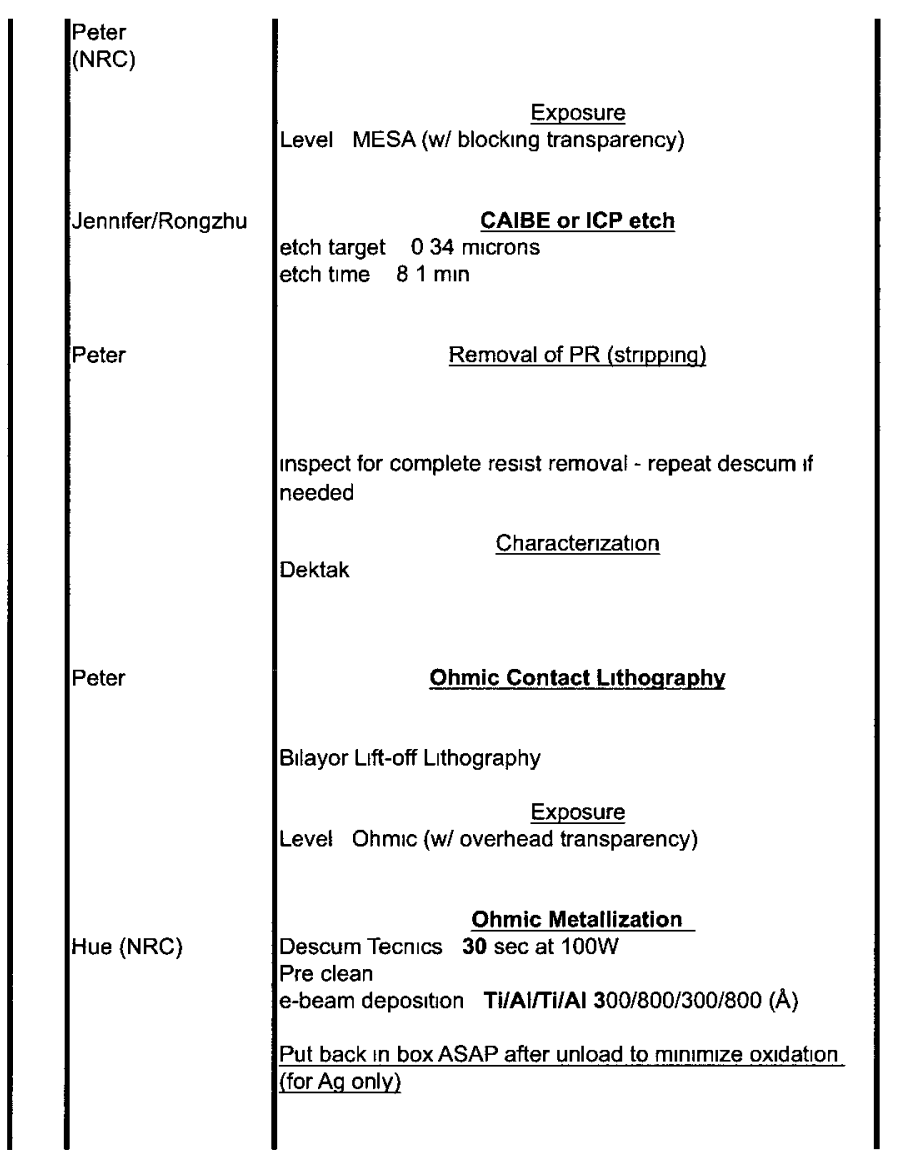


484L - no NRC details

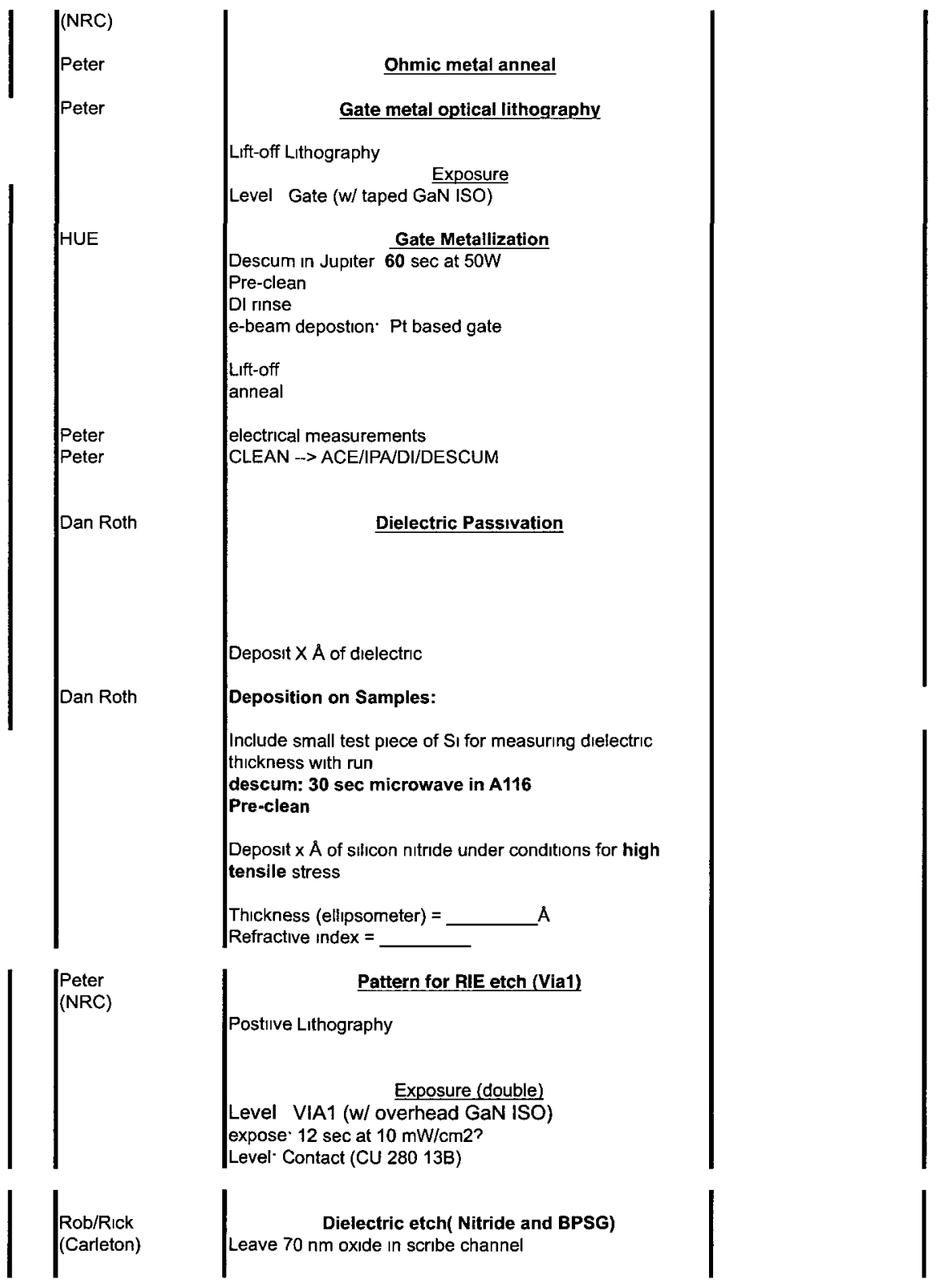

Page 6 
484L - no NRC detarls

$\mid$

RIE etch 05 um BPSG

Gas $15 \%$ O2 + $85 \%$ CHF3

RF POWER $350 \mathrm{~W}$

Pressure $150 \mathrm{mTorr}$

rate $80 \mathrm{~nm} /$ mınute

tıme -5 mınutes, $30 \mathrm{sec}$

actual time- 
484L - no NRC detalls

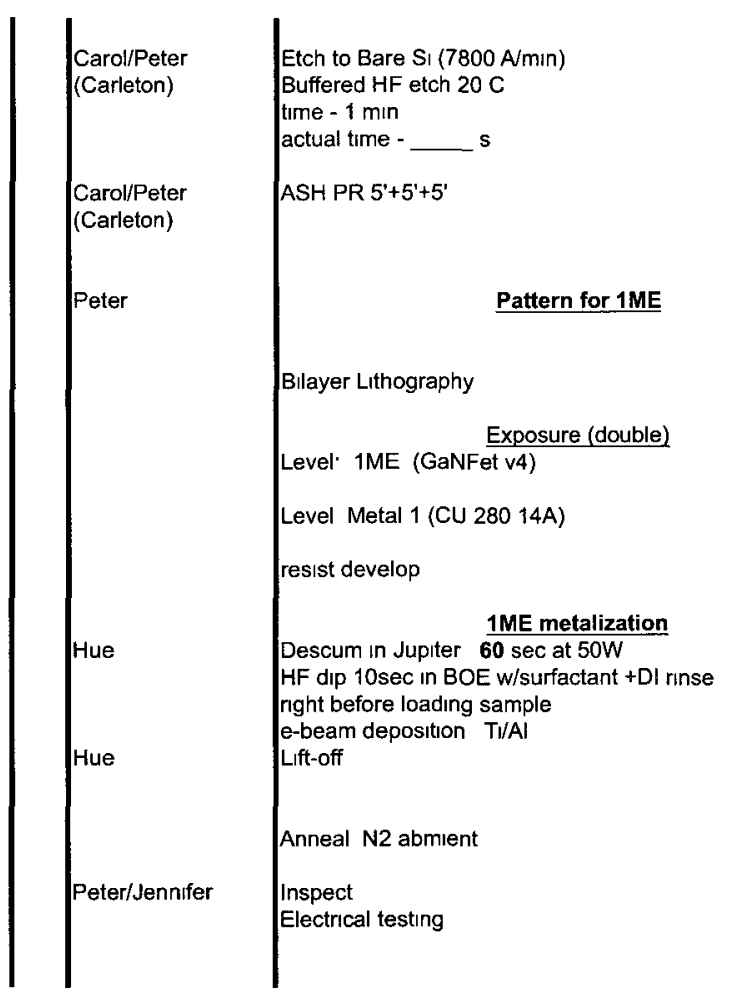

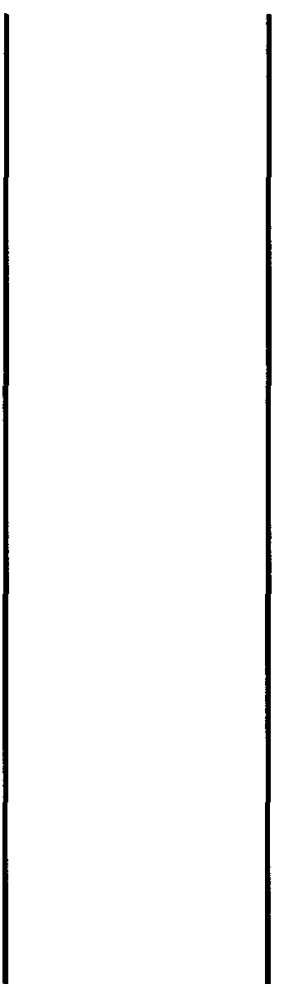

Page 8 


\section{Appendix D}

\section{CV calculations}

This appendix give the mathematical formalism behind the CV analysis used to determine the threshold voltage of the MOS capacitors on test samples.

To determine the capacitance of the $\mathrm{CV} \operatorname{dot}\left(C_{O X}\right)$, we have:

$$
C_{O X}=\frac{A \epsilon_{O X}}{t_{O X}}
$$

where $\epsilon_{O X}$ is the permittivity of the oxide and $t_{O X}$ is the oxide thickness, and A is the area of the capacitor.

The CV measurement can be modelled as two capacitances in series: the oxide capacitance $\left(C_{O X}\right)$ and semiconductor capacitance $\left(C_{S}\right)$, which is variable. In the accumulation region, where the capacitance is at its maximum, the total capacitance is just $C_{O X}$ whereas in other areas, it is the series combination of the two, given by:

$$
C_{G}=\frac{C_{O X} C_{S}}{C_{O X}+C_{S}}
$$

where $C_{G}$ is the total capacitance.

The depletion layer maximum $\left(W_{t}\right)$ can then be determined by extracting the value $C_{\min }$ from the CV curve, the flat region at which the capacitance is at a minimum: 


$$
W_{t}=\sqrt{\frac{\left.4 \epsilon_{S \imath} \phi_{b}\right)}{q N_{A}}}
$$

where $n_{\imath}$ is the intrinsic silicon doping, $N_{A}$ is the acceptor concentration (note p-type silicon is implied here), $\epsilon_{S 2}$ is the silicon permittivity and $q$ is the electron charge. $\phi_{b}$ is given as

$$
\phi_{b}=\frac{K T}{q} \ln \left(\frac{N_{A}}{n_{\imath}}\right)
$$

At full inversion of the layer, $C_{S}$ can be expressed as:

$$
C_{S}=\frac{A \epsilon_{S \imath}}{W_{t}}
$$

Using equation D.5 in combination with D.3 and D.2, a transcendental equation can be achieved for the point at which $C_{G}$ is equal to the minimum capacitance and hence the width of the depletion region is at it's maximum and we can use $w_{t}$. This equation is given as:

$$
\ln \left(\frac{N_{A}}{n_{\imath}}\right)-\frac{q}{\epsilon_{S \imath}}\left(\frac{q}{k T}\right)\left(\frac{\epsilon_{S \imath}}{\epsilon_{O X}}\right)^{2}\left[t_{o x} \frac{C_{O X}}{C_{m \imath n}}-1\right]^{2}=0
$$

$N_{A}$ is subsequently determined by solving using Newton's method.

The threshold voltage can be determined using the following standard equation for threshold voltage.

$$
V_{t}=V_{f b}+2 \phi_{b}+\frac{\sqrt{2 \epsilon_{S \imath} q N_{A} 2 \phi_{b}}}{C_{O X}}
$$

